\title{
A Computational Approach to the Quantification of Animal Camouflage
}

by

\author{
Derya Akkaynak
}

B.S., Aerospace Engineering, Middle East Technical University, Ankara (2003)

M.S., Aeronautics and Astronautics, Massachusetts Institute of Technology (2005)

Submitted to the Department of Mechanical Engineering and the

Joint Program in Applied Ocean Science and Engineering

in partial fulfillment of the requirements for the degree of

Doctor of Philosophy in Mechanical and Oceanographic Engineering

at the

MASSACHUSETTS INSTITUTE OF TECHNOLOGY

and the

WOODS HOLE OCEANOGRAPHIC INSTITUTION

June, 2014

(C) Derya Akkaynak. All rights reserved.

The author hereby grants to MIT and WHOI permission to reproduce and to distribute publicly copies of this thesis document in whole or in part in any medium now known or hereafter created.

Author

Joint Program in Oceanography/Applied Ocean Science and Engineering MIT/WHOI

May 23, 2014

Certified by

Dr. Ruth E. Rosenholtz

Thesis Supervisor, Principal Research Scientist, MIT

Accepted by....

Professor Henrik Schmidt

Chair, Joint Committee for Applied Ocean Science and Engineering, MIT/WHOI

Accepted by

Professor David E. Hardt

Graduate Officer, Department of Mechanical Engineering, MIT 


\title{
A Computational Approach to the Quantification of Animal Camouflage
}

by

\author{
Derya Akkaynak
}

Submitted to the Department of Mechanical Engineering and the Joint Program in Applied Ocean Science and Engineering on May 23, 2014, in partial fulfillment of the requirements for the degree of Doctor of Philosophy in Mechanical and Oceanographic Engineering

\section{Abstract}

Evolutionary pressures have led to some astonishing camouflage strategies in the animal kingdom. Cephalopods like cuttlefish and octopus mastered a rather unique skill: they can rapidly adapt the way their skin looks in color, texture and pattern, blending in with their backgrounds. Showing a general resemblance to a visual background is one of the many camouflage strategies used in nature. For animals like cuttlefish that can dynamically change the way they look, we would like to be able to determine which camouflage strategy a given pattern serves. For example, does an inexact match to a particular background mean the animal has physiological limitations to the patterns it can show, or is it employing a different camouflage strategy (e.g., disrupting its outline)? This thesis uses a computational and data-driven approach to quantify camouflage patterns of cuttlefish in terms of color and pattern. First, we assess the color match of cuttlefish to the features in its natural background in the eyes of its predators. Then, we study overall body patterns to discover relationships and limitations between chromatic components. To facilitate repeatability of our work by others, we also explore ways for unbiased data acquisition using consumer cameras and conventional spectrometers, which are optical imaging instruments most commonly used in studies of animal coloration and camouflage. This thesis makes the following contributions: (1) Proposes a methodology for scene-specific color calibration for the use of RGB cameras for accurate and consistent data acquisition. (2) Introduces an equation relating the numerical aperture and diameter of the optical fiber of a spectrometer to measurement distance and angle, quantifying the degree of spectral contamination. (3) Presents the first study assessing the color match of cuttlefish (S. officinalis) to its background using in situ spectrometry. (4) Develops a computational approach to pattern quantification using techniques from computer vision, image processing, statistics and pattern recognition; and introduces Cuttlefish $72 \times 5$, the first database of calibrated raw (linear) images of cuttlefish.

Thesis Supervisor: Ruth Rosenholtz

Title: Principal Research Scientist 
Bitirdiğimi göremeyen anneme 


\section{Acknowledgments}

Earning a doctoral degree from MIT is no doubt special, but for me, the most valuable part of this journey was having worked with Ruth Rosenholtz. I must have looked like a wrinkled shirt when I showed up at her door. After reading the first draft of our first paper, she said: "Good, now add your research questions to the introduction". I remember thinking "What? I did all this work, now I have to come up with questions?" Without any criticism, judgment or impatience, she ironed every single wrinkle. She never tried to change me; on the contrary, accepted and appreciated everything about me -including some distracting interests I had- and gave me all the room I needed to grow. I am forever grateful. It was an honor to work with her.

If I am receiving the title "Doctor" with this thesis, my husband Elron Yellin needs to receive "Angel" for enduring a very rocky six years. Not only did he have to manage me while I was constantly under stress, but he also put his life on hold so I could pursue my lifelong dream of becoming an oceanographer. Thank you, Elron.

Being affiliated with three research institutions, I made many amazing friends, which also meant I could rant three times about the same subject in one day. Justine Allen initially became my "bff" as a part the scenario we developed to escape Turkish Coast Guard in case we got caught diving in a no-dive zone. We never got caught but the rest of the scenario played out. Kendra Buresch and Kim Ulmer taught me life was too short to not to have an umbrella in my drink, and a drink by the beach Wednesdays at 4 PM made a good tradition. Lydia Mäthger was an amazing mentor who taught me more than half of the skills I learned during my PhD. Having had so much in common, I am sad not to have worked more closely with Liese Siemann, but I loved hearing the stories of her albino hedgehog. George Bell, Robyn Crook and CC Chiao made our lab a fun and significantly more productive place. Last but not least, Roger Hanlon, of course, got me interested in applying to the MIT/WHOI Joint Program in the first place, and took me on many dive trips during which we chased cuttlefish and octopus with the strangest optical instruments. Thanks to him, I even learned some biology, and punctuation.

Every stressed and moody grad student needs a younger brother to torture, and I was lucky Shaiyan Keshvari happened to be at the right place at the right time for me to do just that. With Lavanya Sharan and Erik Hemberg, I took full advantage of the athletics studio in our building and worked my way up to a tripod headstand in a year. Krista Ehinger made the ultimate sacrifice a grad student can make: she selflessly gave up the desk with the window so I could have it. Phillip Isola spent countless hours showing me computer vision tricks but in turn he got to see exclusive photos of camouflaged cuttlefish, which he was fascinated by, so I think we're even. When Bei Xiao moved away, my life was immediately $90 \%$ duller, but somehow I got more work done. MIT friends Alvin Raj, Nat Twareg, Jie Huang, Stella Jia, Wenzhen Yuan, Zhengdong Zhang and Honghua Chang and John Canfield all made me look forward to coming to the office every day, especially on Mondays when we had lab lunch and fun conversations. Rui Lui is the only international celebrity I have known in person. I must thank Prof. Ted Adelson separately; I am always inspired by his ability to explain complex concepts in simple, 
easy-to-digest parts. During one lab lunch he said: "I don't like math, I don't like programming, I don't like reading papers", and I thought that was very humbling coming from a scientist and inventor whose workds had been cited more than 30,000 times.

At Coffee O', on a stinky fishing boat in Alaska, "feeding the fish" over the Gulf Stream or surrounded by penguins in Antarctica, I have some unforgettable scenes in my head with oceanographer friends Clay Kunz, Chris Murphy, Jeff Kaeli, Geoff Gorman, WuJung Lee, Heather Beem, Audrey Maertens, Kalina Gospodinova, Ankita Jain, Nick Loomis, Stephanie Petillo, Toby Schneider and Sheida Danesh. I am looking forward to making future memories.

I am thankful for the support I received from WHOI from Jim Preisig, Tim Stanton, Dana Yoerger, Andy Bowen, Meg Tivey and Chris Reddy. Judy Fenwick, Julia Westwater, Valerie Caron, Marsha Gomes and Leanora Fraser were never tired of helping. At MIT, Ronni Scwartz and Leslie Regan gladly answered about fifty emails I sent per day. I wholeheartedly believe that the mechanical engineering department can keep its doors open thanks to Leslie. Lonny Lippsett gave me the inspiration (and the strategy) to write a paper in 7 days; thanks to him I had fun writing this thesis, too.

I would also like to thank my thesis committee members: John Leonard, Charlie Chubb, Aran Mooney and Norm Farr, for providing great feedback on my research and encouragement along the way.

Finally, I thank my dad and my sister, for their love and support and always being there for me. 


\section{Contents}

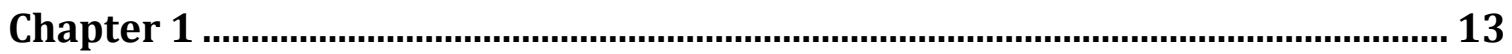

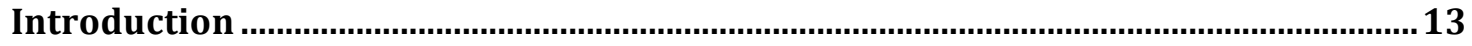

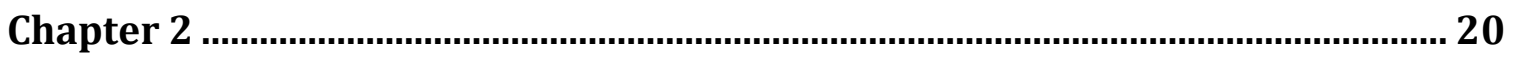

Unbiased data acquisition: Commercial-off-the-shelf digital cameras ........................20

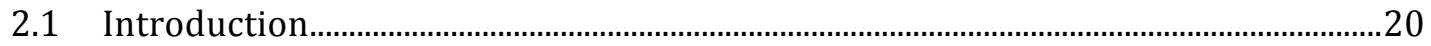

2.2 Background and related work ..........................................................................................22

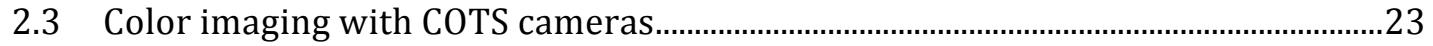

2.4 Image formation principles ……………………………………………………………...23

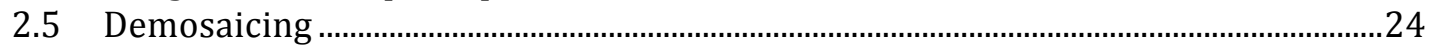

2.6 White balancing ……………………………………………………………………………....25

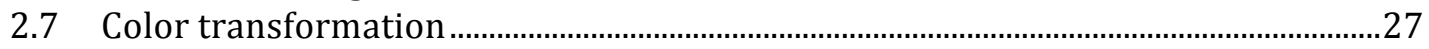

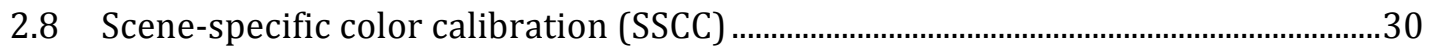

2.9 Examples of the use of COTS cameras for scientific imaging …………………….... 33c

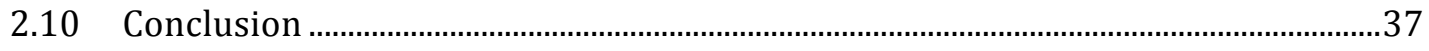

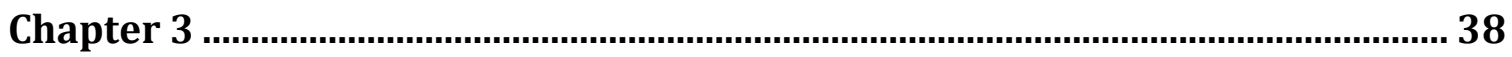

Unbiased data acquisition: Spectrometers ............................................................38

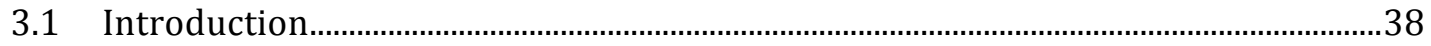

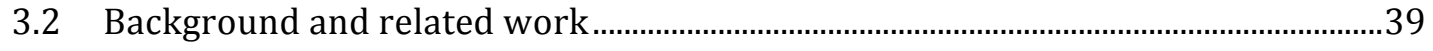

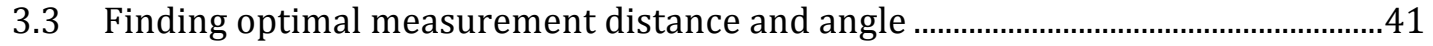

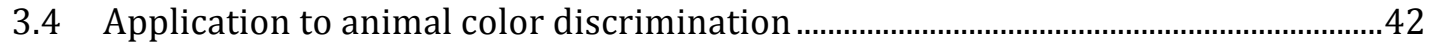

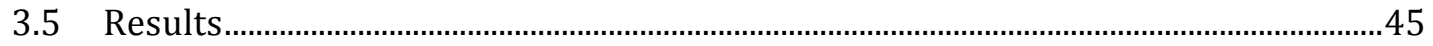

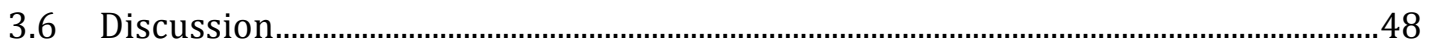

3.7 Conclusion ......................................................................................................................4

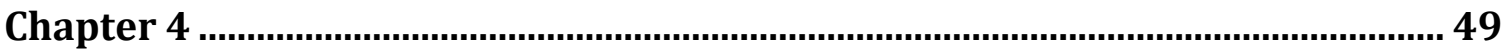

Quantification of cuttlefish camouflage (S. officinalis): a study of color and

luminance using in situ spectrometry.........................................................................49

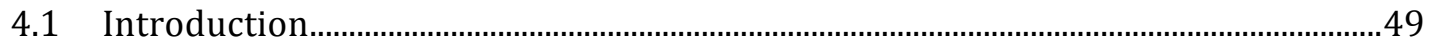

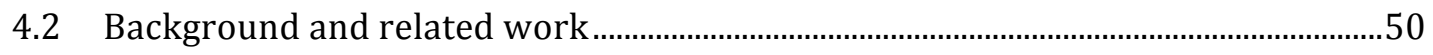

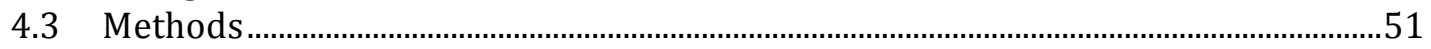

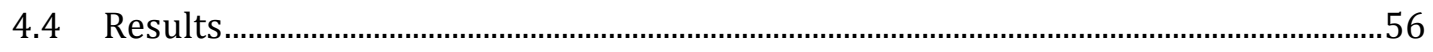

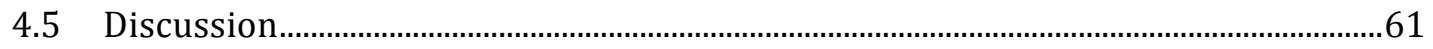

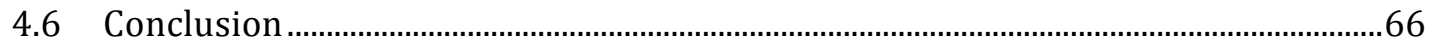

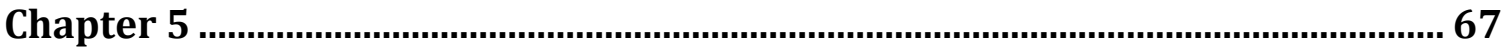

Image-based quantification of cuttlefish (S.officinalis) camouflage patterns...........67

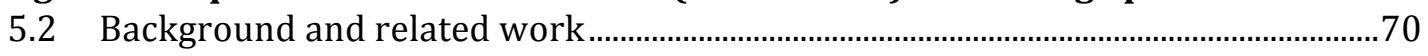

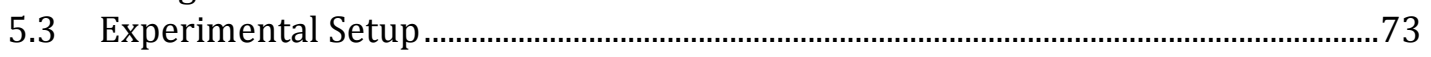

5.4 What is the relationship between the levels of expression of chromatic

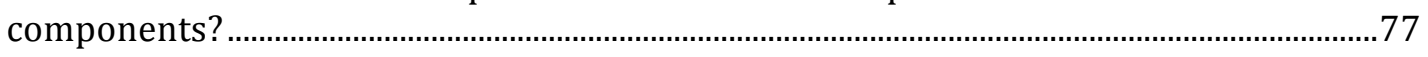

5.5 Do the space of camouflage patterns form a continuum, or are there a discrete

number of clusters? If so, how many?...................................................................................87

5.6 Do cuttlefish express all possible patterns their components are capable of forming, or do they only show a subset? If so, which ones?.................................................90

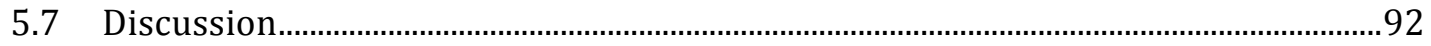

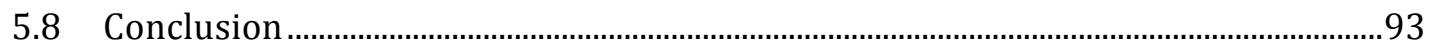

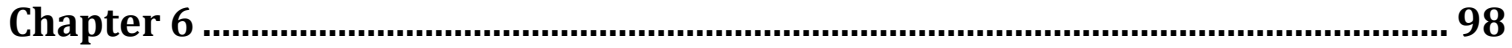




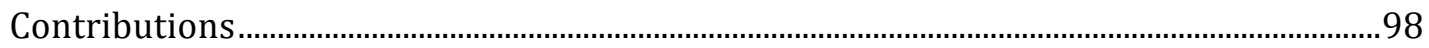

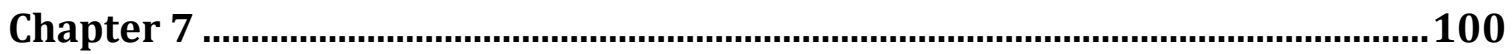

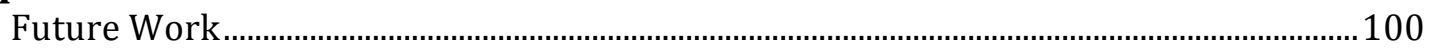

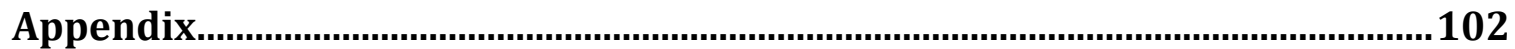

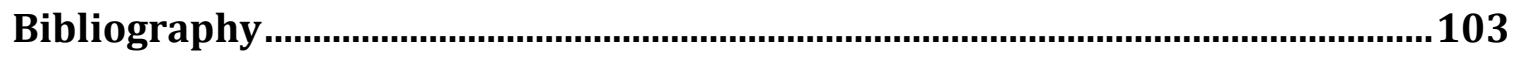




\section{Chapter 1}

\section{Introduction}

Evolutionary pressures have led to some astonishing camouflage strategies in the animal kingdom. One kind of spittlebug evolved to look like bird droppings. Some fish in Amazonian waters adopted the appearance of fallen leaves, and spend all daylight hours motionless on the riverbed (Stevens and Merilaita 2009). In the tropics, brightly colored wing patterns of many butterflies send warning signals to predators indicating that these prey are toxic and should not be consumed. Some of these butterflies are perfectly palatable; they are only mimicking the appearance of their toxic cousins who live nearby (Boggs et al. 2003). Cephalopods like cuttlefish and octopus, which lost their hard outer shell millions of years ago, now depend on camouflage for survival, and have mastered a rather unique skill: they can rapidly adapt the way their skin looks in color, texture and pattern, blending in with their visual backgrounds (Hanlon and Messenger 1996; Messenger 2001b) - a trait noted in Aristotle's Historia Animalium (Aristotle 1910), and grossly exaggerated in many Greek myths. But it is not only their ability to dynamically change their looks that makes cephalopod camouflage impressive; they match their surroundings in color and pattern without actually sensing color with their eyes (Mäthger et al. 2006; Marshall and Messenger 1996). Figure 1.1 shows a small subset of the many different body patterns of cuttlefish we observed during the field season of 2011 in Urla, Turkey.

One immediately wonders their capabilities: "How many different camouflage patterns can cuttlefish show?" "How well does one pattern camouflage the cuttlefish compared to another it could have shown?" "Does each of these patterns fool a different aspect of the predator's visual system?" These are arguably the most important questions in the study of biological camouflage today, generalizing to animals other than cuttlefish. We can best investigate such questions using a comprehensive approach that takes into account the properties of the natural environment camouflage is displayed against, the ambient light field

\section{What is camouflage?}

There are many ways to camouflage without having a body pattern that exactly matches, or resembles a particular background. In biology, camouflage is an umbrella term that describes all strategies used for concealment, including body patterning and coloration, mimicking behavior, and motion (Stevens and Merilaita 2009). While there is no widespread consensus on the definitions of camouflage in the biology community, they are often described based on their function or strategy: i.e. the function of the evolutionary adaptation, such as breaking up form; as opposed to the strategy, the specific perceptual processes targeted, e.g. does the pattern disrupt normal edge detection mechanisms? In this thesis, we are most interested in the crypsis function, which aims to initially prevent detection and includes strategies of background matching, distractive markings, disruptive coloration, self-shadow concealment, obliterative shading and flickerfusion camouflage. Figure 1.2 shows examples of camouflage strategies associated with the crypsis function. and the visual system of the observer(s) viewing the camouflage.

To interpret a scene with a camouflaged animal--in a laboratory setting or in the wild- we would like to be able to describe the animal's camouflage quantitatively, so we can compare certain properties of its pattern to those of its environment. For example, we would like to be able to determine how closely the colors of the animal's body pattern 
match the colors of its background. If we have a model representing the visual system of a predator, we might even be able to assess whether the animal's colors are distinguishable from those of its background in the eyes of that predator. In the same way, we can investigate whether there are any similarities between the spatial composition of a pattern (e.g., lines, splotches, dots, etc.) and the features found in its natural background. Two pre-conditions must be met before undertaking such quantitative analyses. First, the data representing the camouflage scene must be acquired in an unbiased fashion, using the appropriate calibrated instrument(s). In the field of animal coloration and camouflage, most commonly used instruments for data acquisition are consumer cameras, conventional spectrometers and multi/hyper-spectral imagers. Second, the methodology used for pattern quantification must be objective, free of subjective input from human observers, to facilitate repeatability of work by other researchers.

In this thesis, we use a data-driven and computational approach to quantify camouflage patterns of European cuttlefish (Sepia officinalis) using techniques from computer vision, image processing, statistics and pattern recognition. For cuttlefish (and other cephalopods such as squid and octopus), the body pattern, describing the overall appearance of the animal at any given moment, consists of chromatic (e.g., light or dark), textural (e.g., smooth or rough), postural (e.g., skin papillae and arm postures) and locomotor (e.g., buried or hovering) components, all of which can be combined in many ways (Hanlon and Messenger 1996; Messenger 2001b). The visual nature of cephalopod communication and their versatility have led to thinking of the production of body patterns as behaviors (Packard and Hochberg 1977) much like feeding, foraging, reproduction and communication. We utilize images of camouflage scenes collected with both consumer cameras and conventional spectrometers. To ensure our analysis of camouflage meets the necessary pre-conditions mentioned above, we first explore ways of acquiring unbiased data using commercial-off-the-shelf cameras (Chapter 2) and conventional spectrometers (Chapter 3 ). Then, we investigate biologically important questions regarding cuttlefish camouflage. In Chapter 4, we present the first study that assesses color match of cuttlefish in the eyes of its predators, using spectral data collected in situ. Quantifying the color matching abilities of cuttlefish is important because cuttlefish are colorblind (Marshall and Messenger 1996; Mäthger et al. 2006). In addition, the properties of ambient light that penetrates to cuttlefish habitats underwater generally makes all objects appear blue or green, except for those at very shallow depths (Åhlén 2005; Jerlov 1976; Akkaynak et al. 2011; Smith and Baker 1978; Vasilescu 2009). What degree of color match is sufficient to fool the visual systems of predators in such chromatically limited underwater environments?

After investigating color match, we move on to the analysis of entire body patterns of cuttlefish. This is a difficult problem because models of visual pattern perception for fish predators do not exist; and therefore we can only approach this problem from the point of view of the human visual system. Since cuttlefish can dynamically and rapidly change the way their body pattern looks, it is not always straightforward to determine which camouflage strategy their pattern might serve. If they do not display a good resemblance to their backgrounds, is it because they are using a different camouflage strategy (e.g., disruptive coloration, see Figure 1.2), or because they have physiological limitations that prevent them from generating a pattern with a good match to that background? We 
investigate the camouflage pattern generating capabilities of cuttlefish from calibrated photographs in Chapter 5. Understanding limitations to the patterns cuttlefish show can give us insights into determining whether cuttlefish are capable of employing more than one camouflage strategy. Even though it has not been experimentally proven for cephalopods, Hanlon and colleagues present evidence of disruptive coloration in two different species of cuttlefish (Hanlon et al. 2009; Hanlon et al. 2013). If cuttlefish can indeed employ different camouflage strategies, when we investigate the structure of the space their patterns form, we might expect to observe well-separated, discrete number of clusters, instead of a continuum. Such analyses can contribute to the ongoing research regarding how many camouflage patterns cuttlefish can show, and in turn, help answer how many total camouflage patterns there may be in all of animal kingdom.

\section{Imaging camouflage patterns}

The tools used to study camouflage, particularly to image animals and their backgrounds are important because the appearance of a camouflage scene may be different when viewed by a non-human animal's visual system. Indeed, our lack of consideration or knowledge of the visual mechanisms of the relevant observers is thought to be one of the major obstacles hindering full understanding of camouflage strategies (Stevens and Merilaita 2009). We know less about the visual systems of animals than we do about humans' (Stevens et al. 2007; Mäthger et al. 2008), but taking into account the visual capabilities of the observer(s) viewing a camouflage pattern is important because analyzing color signals from the point-of-view of humans and making inferences about their appearances to animals often produces erroneous conclusions. This is because animal visual systems differ from ours in important ways. For example, birds have four photoreceptors, one more than humans with normal color vision. In addition, they are sensitive to the ultraviolet (UV) part of the electromagnetic spectrum (Hart et al. 1998), to which we are not. In a recent publication, Stoddard and Stevens (Stoddard and Stevens 2011) showed that some common cuckoo eggs (which are laid in nests of other species and often hatched and raised by unsuspecting host birds) appeared to have a good color match to the host eggs when viewed by humans, but had clear and quantifiable differences when modeled in avian color space. Their study highlights a key point: the optical instrument that will be used to image camouflage pattern must be capable of recording information in the parts of the electromagnetic spectrum to which relevant observers are sensitive.

Three kinds of optical instruments are used for imaging animal patterns: consumer cameras (also known as RGB cameras), spectrometers and hyper-spectral imagers (Akkaynak 2014). Among these, hyper-spectral imagers provide the most comprehensive data because in addition to recording a spatial image, they also densely sample the electromagnetic spectrum, creating an image of dimensions $N x M x P$. These dimensions correspond to height $(N)$, width $(M)$ and the number of spectral samples $(P$, typically between 70-100). Despite being ideal instruments for imaging animal patterns in color, hyper-spectral imagers are least commonly used for animal coloration studies because of their high cost and physical bulkiness. In addition, their slow imaging times that make it difficult to photograph moving objects like animals in the wild. Consumer cameras, on the other hand, provide a compact and affordable alternative to hyper- 
spectral imagers because they also do spatial imaging; however they are limited in color capture capabilities.
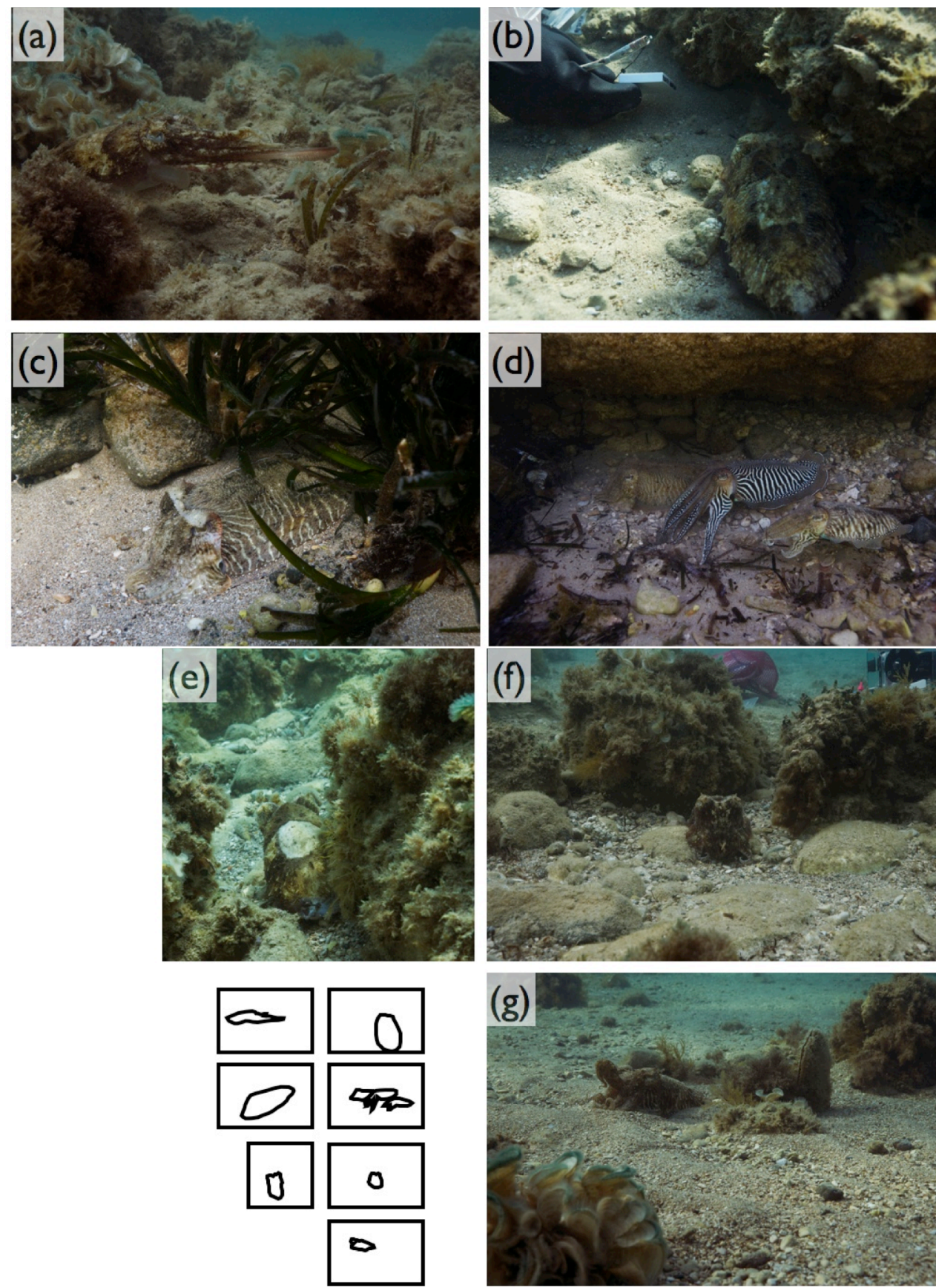

Figure 1.1 The versatility of cuttlefish, and its remarkable ability to combine pigments with textural elements and body postures makes these animals perfect models for the study of camouflage. Here we show a subset of body patterns, used for communication and camouflage, that we observed in cuttlefish's (S.officinalis) natural habitat on the Aegean coast of Turkey. Image credits: Derya Akkaynak, Justine J. Allen \& Roger T. Hanlon. 
By design, consumer cameras sample the electromagnetic spectrum narrowly, creating images of dimensions $N x M x 3$, and the parts of the spectrum they record data from are chosen to overlap with the sensitivities of the human visual system (Akkaynak et al. 2014; Stevens et al. 2007). Even when it is desired or acceptable to capture a photograph just in the visible range, there are a number of manual processing steps required before camera-dependent colors can be transformed into a camera-independent color space, which is a minimum requirement to facilitate repeatability of work by others in the context of scientific applications. In the field of animal coloration and camouflage, the limitations of consumer cameras and the need to manually process their images were generally overlooked until the work of Stevens et al. (2007), who emphasized that incamera processed photographs (e.g., images in jpg format) may contain artifacts that cannot be removed, compromising data quality. In this work, we expand their study that focused on consistency of colors captured with consumer cameras, and introduce a method to also increase the accuracy of color capture using consumer cameras. We call this method scene-specific color calibration, and describe it in detail in Chapter 2.

To overcome the color limitations of consumer cameras, conventional spectrometers with optical fibers are frequently used in their place, or in tandem, to measure colors from an animal's habitat, nest, eggs, body parts, skin, fur and plumage (Akkaynak 2014). Spectrometers sample the electromagnetic spectrum densely with the capability to record information outside of the visible range. However, the image they record is a single pixel big (i.e., has dimensions $1 \times 1 \times 1 \times P$ ), requiring multiple measurements on a carefully gridded setup, and a complex reconstruction scheme to capture spectra for the entire layout of a pattern. In addition, depending on the optical properties of a fiber, it may not be possible to resolve spectra from features that are very small without colors from neighboring features mixing in. For features that are large enough, recording uncontaminated spectra demands the positioning of the optical fiber as close as possible to surface being measured, without touching it. This is standard practice in laboratory setups but not easily accomplished in field studies because it is challenging to get the fiber very close to freely behaving animals in the wild. In Chapter 3, we investigate what kinds of camouflage assessment errors contaminated spectra can lead to if the surveyor fails to get very close to the feature being measured.

\section{Quantifying camouflage patterns}

Given a camouflage pattern, we would like to be able to determine which aspects of the visual background it may match, or which camouflage strategy it serves. This is not straightforward because quantitative models describing camouflage strategies do not exist. Mäthger and colleagues (2008) were to first to quantitatively analyze the color matching abilities of cuttlefish. They measured reflectance spectra from ten points on animals and background substrates in the laboratory and used normalized Euclidean distance to quantify spectral difference. They found that cuttlefish showed a better spectral match to natural substrates than they did for artificial substrates, but did not analyze the discriminability of colors according to the visual systems of predators. Chiao and colleagues (2011) also performed a color match assessment in the laboratory, but they used a hyper-spectral imager to capture body patterns. They modeled the visual systems of hypothetical predators and found that the colors cuttlefish produced were indistinguishable in the eyes of predators. In both studies, the natural substrates used 
were sands and gravel. Cuttlefish live in habitats ranging from the Mediterranean to the coral reefs of West Africa, which have a diversity of background substrates that extend beyond sands and gravel including three-dimensional elements like sea grass, pinna, rocks, boulders, etc. We advanced existing work by travelling to one of the natural habitats of cuttlefish in the Aegean Sea and conducting the first in situ spectroscopic survey of color and luminance from cuttlefish skin and substrates from that habitat. Then, we calculated color and luminance contrast using the Vorobyev-Osario receptor noise model (Vorobyev and Osorio 1998), which allowed us to assess the discriminability of colors in the eyes of hypothetical di- and tri-chromat predators (Chapter 4). In this field study, we focused only on quantifying spectral discriminability, omitting spatial configurations of body patterns, which we investigate in Chapter 5 .

To compare one camouflage pattern with another and quantify the differences between them, it is important to use a dataset that is free of instrument bias, and an analysis method that does not contain subjective judgments. To facilitate repeatability of our work by others, prior to our analysis of overall camouflage patterns, we created Cuttlefish $72 \times 5$, the first database consisting of linear images of camouflaged cuttlefish. It was important to establish this database before asking biological camouflage questions because previous work describing animal patterns and coloration often based their analyses on images in jpg format. Such images are standard outputs of consumer cameras and have been processed and compressed in ways that they no longer maintain a linear relationship to scene radiance (Stevens et al. 2007; Akkaynak et al. 2014). This means that they may not accurately represent the pattern that was photographed. In addition, earlier studies that describe camouflage patterns involved subjective judgments by human observers. While assessments of patterns by human observers is quick and does not require any specialized instruments, it is inherently subjective and the resulting qualitative descriptions often vary between observers, causing low repeatability (Stevens 2011). Thus, camera bias and processing artifacts were minimized for the images in our database, facilitating repeatability of our results by other researchers. Based on the images in the Cuttlefish $72 \times 5$ database, we focused on three aspects of cuttlefish camouflage patterns and used computational methods to investigate them: the relationship between the level of expression of components in terms of intensity and mottleness; the structure of the camouflage pattern space (e.g., do patterns form discrete clusters or a continuum), and whether cuttlefish can show all of the patterns they are theoretically capable of producing. These questions are investigated in detail in Chapter 5 . 
camouflage, meaning all strategies involved in concealment, including prevention of detection and recognition

crypsis (initially preventing detection):

(a) background matching, where the appearance generally matches the color, lightness, and pattern of one (specialist), or several (compromise) background types

(b) self-shadow concealment, where directional light (which would leave to the creation of shadows) is cancelled out by countershading.

(c) obliterative shading, where countershading leads to the obliteration of three-dimensional form

(d) disruptive coloration, being a set of markings that creates the appearance of false edges and boundaries, and hinders the detection or recognition of an objet's or part of an object's true outline and shape

(e) flicker-fusion camouflage, where markings such as stripes blur during motion to match the color/lightness of the general background, preventing detection of the animal when in motion

(f) distractive markings, which direct the attention or gaze of the receiver from traits that would give away the animal (such as the outline)

masquerade, where recognition is prevented by resembling an uninteresting object, such as a leaf or a stick

motion dazzle, where markings make estimates of speed and trajectory difficult by an observer motion camouflage, movement in a fashion that decreases the probability of movement detection

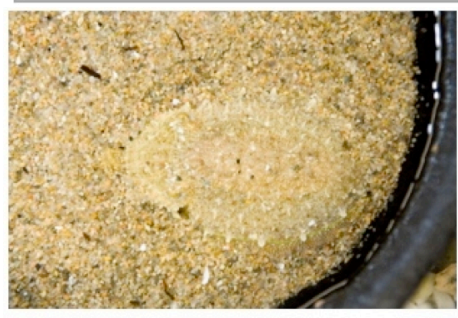

(a)

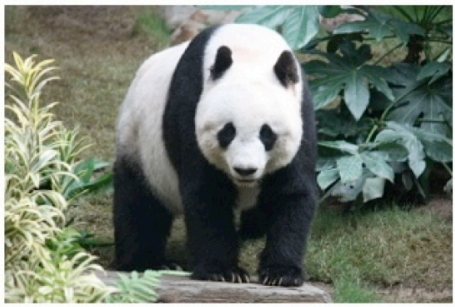

(d)

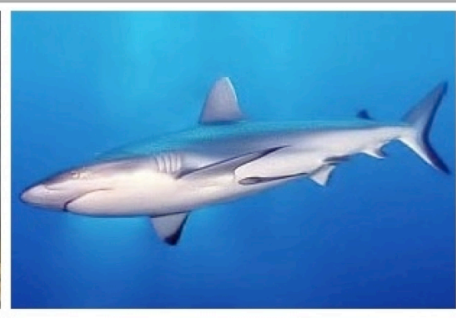

(b)

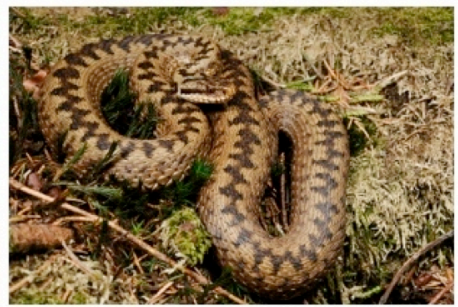

(e)

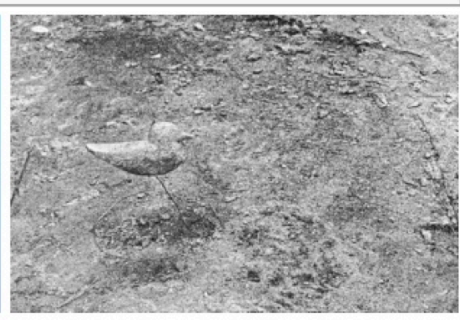

(c)

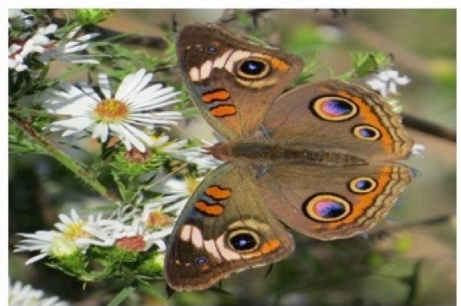

(f)

Figure 1.2 While there is no widespread consensus on the definitions of biological camouflage, Stevens and Merilaita (2009) provide the most comprehensive set of descriptions, which we adopt in this work. (a) Background matching by cuttlefish (b) Counter-shaded grey reef shark (c) Obliterative shading makes one of the two decoy ducks in this photograph disappear (on the right, practically invisible) while the non-countershaded on the left is visible (d) Disruptive pattern of a panda bear (e) The patterning of the common European viper might trigger a flicker-fusion illusion (f) False eye spots serve as distractive markings on this brush-footed butterfly. Image credits: (a): Hanlon Lab, (b)-(f): Wikipedia. 


\section{Chapter 2}

\section{Unbiased data acquisition: Commercial-off-the-shelf digital cameras $^{1}$}

\subsection{Introduction}

State-of-the-art hardware, built-in photo enhancement software, waterproof housings and affordable prices enable widespread use of commercial off-the-shelf (COTS) digital cameras in research laboratories. However it is often overlooked that these cameras are not optimized for accurate color capture, but rather for producing photographs that will appear pleasing to the human eye when viewed on small gamut and low dynamic range consumer devices (Chakrabarti et al. 2009; Seon Joo et al. 2012). As such, use of cameras as black-box systems for scientific data acquisition, without knowledge and control of how photographs are manipulated inside, may compromise data quality and in turn, hinder repeatability. For example, a common color-sensitive use of consumer cameras is in the field of animal coloration and camouflage (Stevens et al. 2007; Pike 2011b). How well do the colors from the skin of a camouflaged animal match the colors in a particular background? For an objective analysis of this question, the photograph of the animal taken by a researcher using a certain make and model COTS camera should be reproducible by another researcher using a different camera. This is not straightforward to accomplish because each camera records colors differently, based on the material properties of its sensor, and built-in images processing algorithms are often make and model specific, and proprietary. Thus, for scientific research, manual processing of COTS camera images to obtain device-independent photographs is crucial. In this chapter, we explore the following question: what are the pre- and post-image acquisition steps necessary so color capture using COTS cameras is consistent and accurate enough to yield photographs that can be used as scientific data? Our goal is to identify the limitations of COTS cameras for color imaging and streamline the manual processing steps necessary for their use as consistent and accurate scientific data acquisition instruments.

\section{A consumer camera}

photograph is considered unbiased if it has a known relationship to scene radiance. This can be a purely linear relationship, or a non-linear one where the non-linearities are precisely known and can be

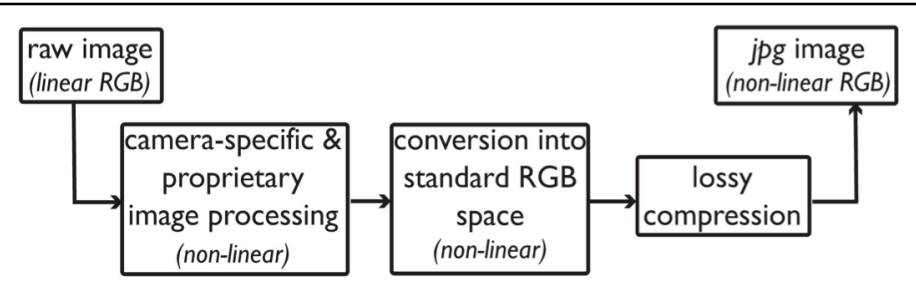

Figure 2.1 Basic image-processing pipeline in a consumer camera. inverted. A linear relationship to scene radiance makes it possible to obtain deviceindependent photographs that can be quantitatively compared with no knowledge of the original imaging system. Raw photographs recorded by many cameras have this desired property (Chakrabarti et al. 2009), whereas camera-processed images, most commonly

${ }^{1}$ Parts of this chapter were previously published. See (Akkaynak et al. 2014) for full reference. 
images in jpg format, do not. For scientific research, obtaining device-independent photographs is crucial because each consumer camera records colors differently. Only after camera-dependent colors are transformed to a standard, camera-independent color space, researchers using different makes and/or models of cameras can reproduce them.

When pixel intensities in photographs are to be used as scientific data, it is important to use raw images, and manually process them, controlling each step, rather than using camera-processed images. In-camera processing introduces non-linearities through make and model-specific and often proprietary operations that alter the color, contrast and white balance of images. These images are then transformed to a non-linear RGB space, and compressed in an irreversible fashion (Figure 2.1). Compression, for instance, creates artifacts that can be so unnatural that they may be mistaken for cases of image tampering (Figure 2.2) (Farid 2011). As a consequence, pixel intensities in consumer camera photographs are modified such that they are no longer linearly related to scene radiance. Models that approximate raw (linear) RGB from non-linear RGB images (e.g., sRGB) exist, but at their current stage they require a series of training images taken under different settings and light conditions as well as ground-truth raw images (Seon Joo et al. 2012).

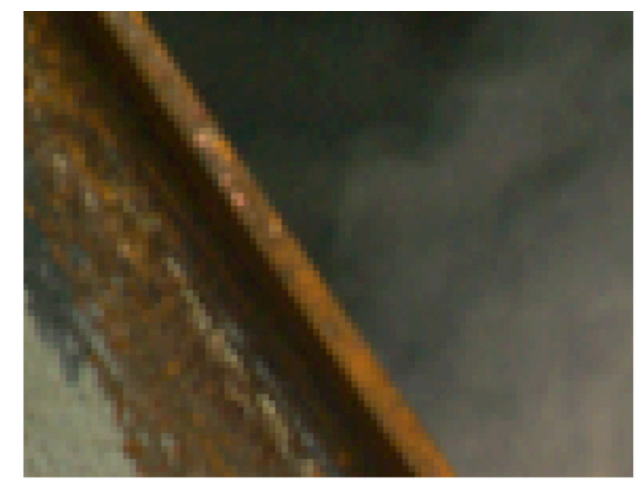

(a)

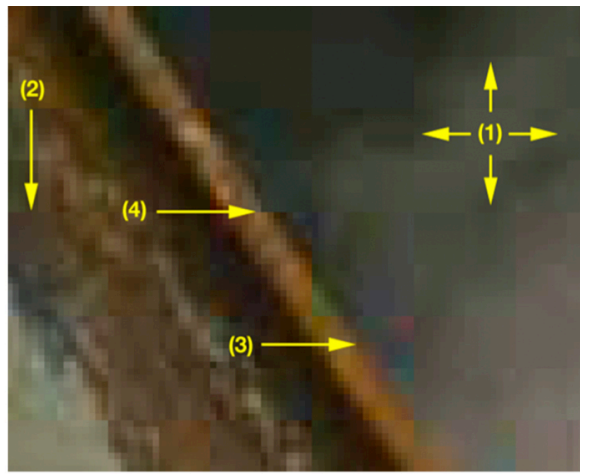

(b)

Figure 2.2 (a) An uncompressed image. (b) Artifacts after jpg compression: 1) grid-like pattern along block boundaries 2) blurring due to quantization 3) color artifacts 4) jagged object boundaries. Photo credit: Dr. Hany Farid. Used with permission. 


\subsection{Background and related work}

The limitations and merit of COTS cameras for scientific applications have previously been explored, albeit disjointly, in ecology (Levin et al. 2005), environmental sciences (De La Barrera and Smith 2009), systematics (McKay 2013), animal coloration (Stevens et al. 2007), dentistry (Wee et al. 2006) and underwater imaging (Åhlén 2005; Akkaynak et al. 2011). Stevens et al. (Stevens et al. 2007) wrote:

“... most current applications of digital photography to studies of animal coloration fail to utilize the full potential of the technology; more commonly, they yield data that are qualitative at best and uninterpretable at worst".

Our goal is to address this issue and make COTS cameras accessible to researchers from all disciplines as proper data collection instruments. We focus on two aspects of color capture using consumer cameras: consistency (Ch. 2.3-2.7) and accuracy (Ch. 2.8). Calibration of consumer cameras through imaging photographic calibration targets (i.e., camera characterization) is a technique well known to recreational and professional photographers for obtaining consistent colors under a given light source. Researchers in fields like image processing, computer vision and computational photography also frequently use this technique (Szeliski 2010; Shirley et al. 2009; Pharr and Humphreys 2010; Joshi and Jensen 2004). However, in the field of animal coloration, the limitations of consumer cameras and the need to manually calibrate them were generally overlooked until the work of Stevens et al. (2007), who emphasized that incamera processed photographs (e.g., images in jpg format) may contain artifacts that cannot be removed, compromising data quality. In the first part of this chapter, we review and streamline the necessary manual processing steps described by Stevens et al. (2007) and others.

In Chapter 2.8, we introduce the idea of scene-specific color calibration and show that it improves color transformation accuracy when a non-ordinary scene is photographed. We define an ordinary scene as one that has colors that are within the gamut of a commercially available color calibration target. Color transformation, which we describe in detail in Chapter 2.7, is done through a transformation matrix that maps device-specific colors to a standard, device-independent space based on known, manufacturer-specified chromatic properties of calibration target patches. This transformation matrix is generally computed through solving a system of equations with using linear least squares regression. Previous work that focused on increasing color transformation accuracy used standard, off-the-shelf calibration targets and investigated methods other than linear least squares regression to minimize the error between captured and desired RGB values. Examples of methods used include non-linear regression (Westland and Ripamonti 2004), constrained least squares regression (Finlayson and Drew 1997), neural networks (Cheung et al. 2004) and interpolation (Johnson 1996). Our approach differs from existing work in that we use the simplest mathematical solution to the system of equations that describe the color transformation (i.e., linear least squares regression), and derive the desired set of RGB value directly from features in the scene instead of commercial photographic calibration targets.

Even though our work is motivated by accurate capture of colors from camouflage patterns of animals, our methodology is applicable to problems in all fields of 
science consumer cameras are used; we demonstrate this in Chapter 2.9 using real scientific problems. Throughout this work, "camera" will refer to "COTS digital cameras", also known as "consumer," "digital still," "trichromatic" or "RGB" cameras. Any references to RGB will mean "linear RGB" and non-linear RGB images or color spaces will be explicitly specified as such.

\subsection{Color imaging with COTS cameras}

Color vision in humans (and other animals) is used to distinguish objects and

surfaces based on their spectral properties. Normal human vision is trichromatic; the retina has three cone photoreceptors referred to as short $(\mathrm{S}$, peak $440 \mathrm{~nm})$, medium (M, peak 545 $\mathrm{nm})$ and long (L, peak $580 \mathrm{~nm})$. Multiple light stimuli with different spectral shapes evoke the same response. This response is represented by three

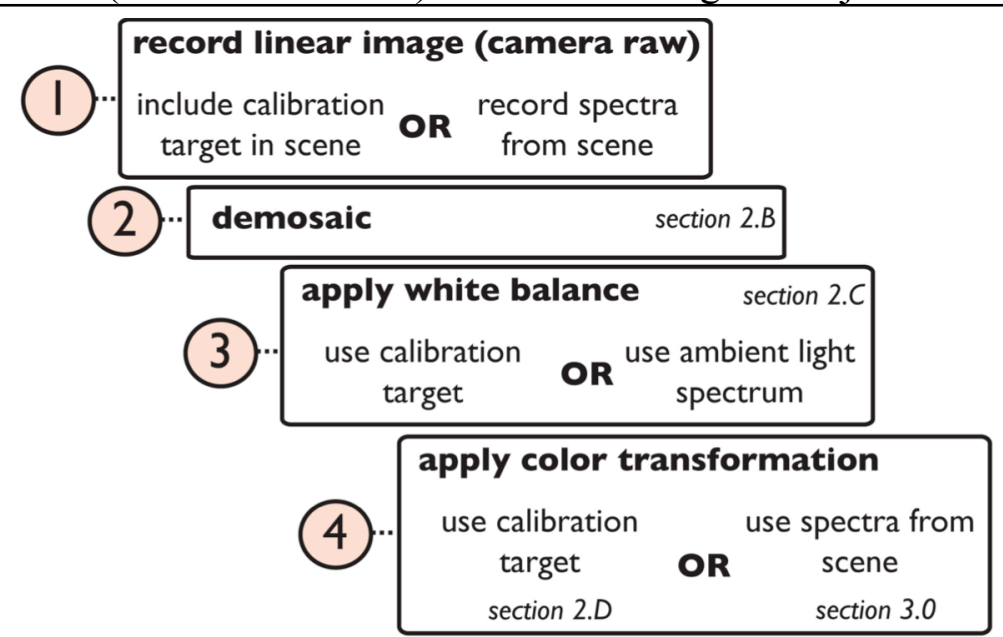

Figure 2.3 The workflow proposed for processing raw images. Consumer cameras can be used for scientific data acquisition if images are captured in raw format and processed manually so that they maintain a linear relationship to scene radiance.

scalars known as tri-stimulus values, and stimuli that have the same tri-stimulus values create the same color perception (Wyszecki and Stiles 2000). Typical cameras are also designed to be trichromatic; they use color filter arrays on their sensors to filter broadband light in the visible part of the electromagnetic spectrum in regions humans perceive as red (R), green $(\mathrm{G})$ and blue $(B)$. These filters are characterized by their spectral sensitivity curves, unique to every make and model (Figure 2.4). This means that two different cameras record different RGB values for the same scene.

Human photoreceptor spectral sensitivities are often modeled by the color matching functions defined for the $2^{\circ}$ observer (foveal vision) in the CIE $1931 \mathrm{XYZ}$ color space. Any color space that has a well-documented relationship to XYZ is called device-independent (Reinhard et al. 2008). Conversion of device-dependent camera colors to device-independent color spaces is the key for repeatability of work by others; we describe this conversion in Ch. $2.7 \& 2.8$.

\subsection{Image formation principles}

The intensity of light recorded at a sensor pixel is a function of the light that illuminates the object of interest (irradiance, Figure 2.5), the light that is reflected from the object towards the sensor (radiance), the spectral sensitivity of the sensor and optics of the imaging system: 


$$
\mathrm{I}_{\mathrm{c}}=\mathrm{k}(\gamma) \cos \theta_{\mathrm{i}} \int_{\lambda_{\min }}^{\lambda_{\max }} \mathrm{S}_{\mathrm{c}}(\lambda) \mathrm{L}_{\mathrm{i}}(\lambda) \mathrm{F}(\lambda, \theta) \mathrm{d} \lambda
$$

Here $\mathrm{c}$ is the color channel (e.g., R, G, B), $S_{c}(\lambda)$ is the spectral sensitivity of that channel, $\mathrm{L}_{\mathrm{i}}(\lambda)$ is the irradiance, $\mathrm{F}(\lambda, \theta)$ is the bi-directional reflectance distribution function and $\lambda_{\min }$ and $\lambda_{\max }$ denote the lower and upper bounds of the spectrum of interest, respectively (Szeliski 2010). Scene radiance is given by:

$$
\mathrm{L}_{\mathrm{r}}(\lambda)=\mathrm{L}_{\mathrm{i}}(\lambda) \mathrm{F}(\lambda, \theta) \cos \theta_{\mathrm{i}}
$$

where $F(\lambda, \theta)$ is dependent on the incident light direction as well as the camera viewing angle where $\theta=\left(\theta_{i}, \phi_{i}, \theta_{r}, \phi_{r}\right)$. The function $\mathrm{k}(\gamma)$ depends on optics and other imaging parameters and the $\cos \theta_{i}$ term accounts for the changes in the exposed area as the angle between surface normal and illumination direction changes. Digital imaging devices use different optics and sensors to capture scene radiance according to these principles (Table $1)$.

Table 1 Comparison of basic properties of color imaging devices

\begin{tabular}{|c|c|c|c|c|}
\hline Device & Spatial & Spectral & Image Size & Cost \\
\hline Spectrometer & $\times$ & $\checkmark$ & $1 \times \mathrm{p}$ & $\geq \$ 2,000$ \\
\hline COTS camera & $\checkmark$ & $\times$ & $\mathrm{n} \times \mathrm{m} \times 3$ & $\geq \$ 200$ \\
\hline $\begin{array}{c}\text { Hyperspectral } \\
\text { imager }\end{array}$ & $\checkmark$ & $\checkmark$ & $\mathrm{n} \times \mathrm{m} \times \mathrm{p}$ & $\geq \$ 20,000$ \\
\hline
\end{tabular}

\subsection{Demosaicing}

In single-sensor cameras the raw image is a two dimensional array (Figure 2.6a, inset). At each pixel, it contains intensity values that belong to one of $R, G$ or $B$ channels according to the mosaic layout of the filter array. Bayer Pattern is the most commonly used mosaic. At each location, the two missing intensities are estimated through interpolation in a process called demosaicing (Ramanath et al. 2002). The highest quality demosaicing algorithm available should be used regardless of its computation speed (Figure 2.6), because speed is only prohibitive when demosaicing is carried out using the limited resources in a camera, not when it is done by a computer.
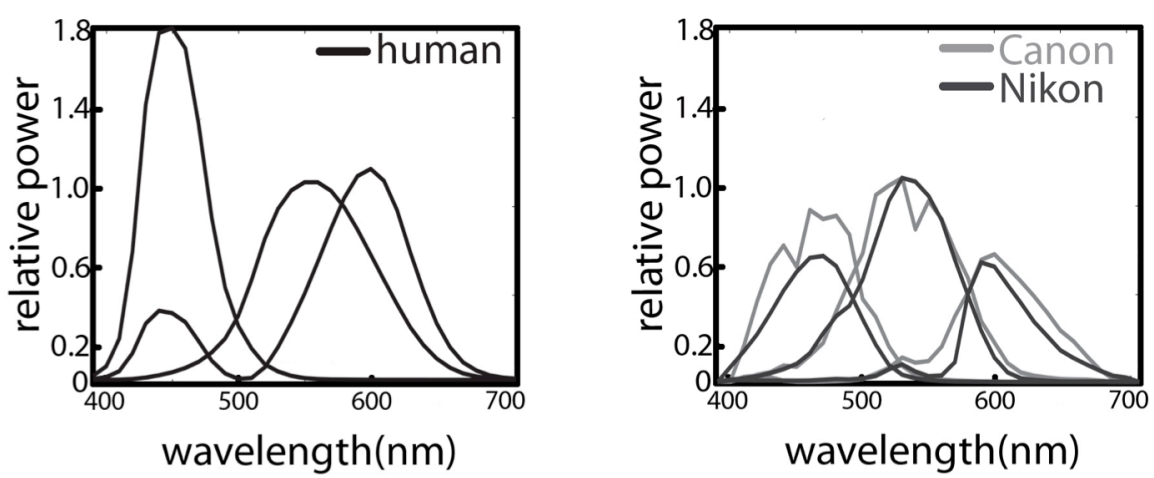

Figure 2.4 Human color matching functions for the CIE XYZ color space for $2^{\circ}$ observer and spectral sensitivities of two cameras; Canon EOS 1Ds mk II and Nikon D70. 


\subsection{White balancing}

In visual perception and color reproduction, white has a privileged status (Finlayson and Drew 1997). This is because through a process called chromatic adaptation our visual system is able to discount small changes in the color of an illuminant, effectively causing different lighting conditions to appear "white" (Reinhard et al. 2008). For example, a white slate viewed underwater would still be perceived as white by a SCUBA diver, even though the color of the ambient light is likely to be blue or green, as long as the diver is adapted to the light source. Cameras cannot adapt like humans, and therefore cannot discount the color of the ambient light. Thus, photographs must be white balanced to appear realistic to a human observer. White balancing often refers to two concepts that are related but not identical: RGB equalization and chromatic adaptation transform (CAT), described below.

light spectrum

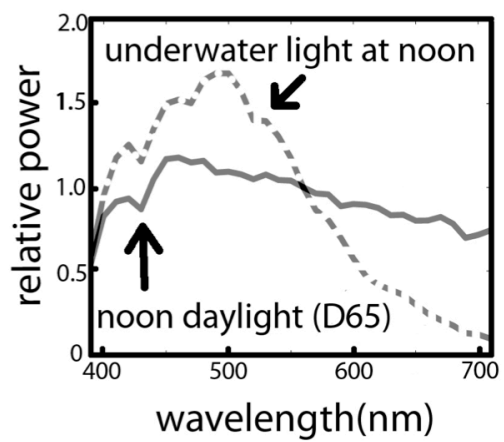

(a)

radiance

noon daylight

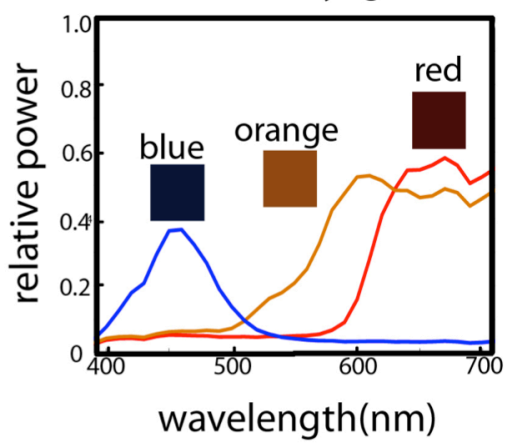

(c)

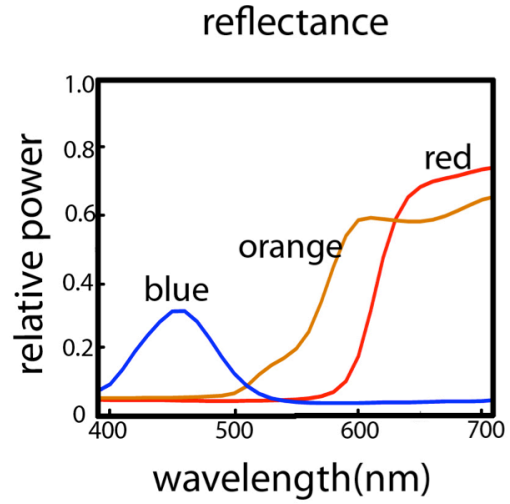

(b)

radiance

underwater light at noon

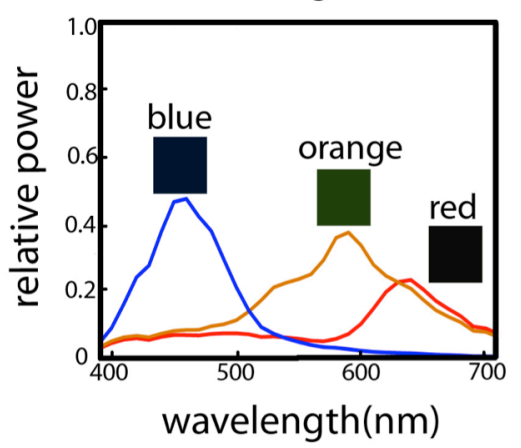

(d)

Figure 2.5 (a) Irradiance of daylight at noon (CIE D65 illuminant) and noon daylight on a sunny day recorded at 3 meters depth in the Aegean Sea. (b) Reflectance spectra of blue, orange and red patches from a Macbeth ColorChecker. Reflectance is the ratio of reflected light to incoming light at each wavelength and it is a physical property of a surface, unaffected by the ambient light field, unlike radiance. (c) Radiance of the same patches under noon daylight on land and (d) underwater.

In scientific imaging, consistent capture of scenes often has more practical importance than capturing them with high perceptual accuracy. White balancing as 
described here is a linear operation modifies photos so they appear "natural" to us. For purely computational applications in which human perception does not play a role, and therefore a natural look is not necessary, white balancing can be done using RGB Equalization, which has less perceptual relevance than CAT, but is simpler to implement (see examples in Ch. 2.9). Here, we describe both methods of white balancing and leave it up to the reader to decide which method to use.

\subsubsection{Chromatic Adaptation Transform (CAT)}

Also called white point conversion, CAT models approximate the chromatic adaptation phenomenon in humans, and have the general form:

$$
V_{\text {destination }}^{X Y Z}=\left[M_{A}\right]^{-1}\left[\begin{array}{ccc}
\rho_{D} / \rho_{S} & 0 & 0 \\
0 & \gamma_{D} / \gamma_{S} & 0 \\
0 & 0 & \beta_{D} / \beta_{S}
\end{array}\right]\left[M_{A}\right] V_{\text {source }}^{X Y Z}
$$

where $\mathrm{V}^{\mathrm{XYZ}}$ denotes the $3 \times \mathrm{N}$ matrix of colors in $\mathrm{XYZ}$ space, whose appearance is to be transformed from the source illuminant (S) to the destination illuminant (D); $M_{A}$ is a $3 \times 3$ matrix defined uniquely for the CAT model and $\rho, \gamma$ and $\beta$ represent the tri-stimulus values in the cone response domain and are computed as follows:

$$
\left.\left[\begin{array}{l}
\rho \\
\gamma \\
\beta
\end{array}\right]_{\mathrm{i}}=\left[M_{A}\right]_{[W P}\right]_{\mathrm{i}}^{X Y Z} \quad i=S, D
$$

Here, WP is a $3 \times 1$ vector corresponding to the white point of the light source. The most commonly used CAT models are Von Kries, Bradford, Sharp and CMCCAT2000. The $M_{A}$ matrices for these models can be found in (Süsstrunk et al. 2000).

original scene

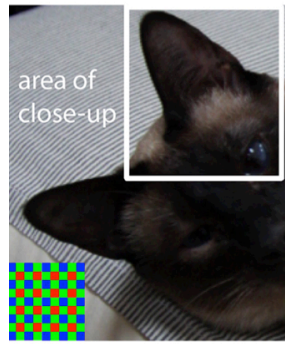

(a) high-quality demosaicing

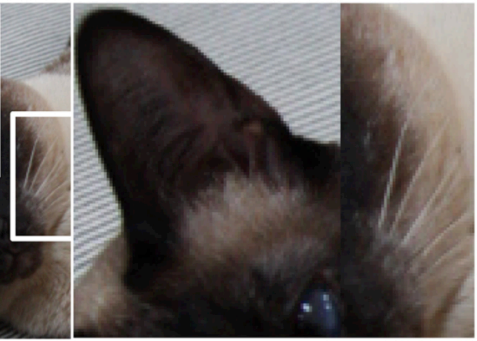

(b) low-quality demosaicing

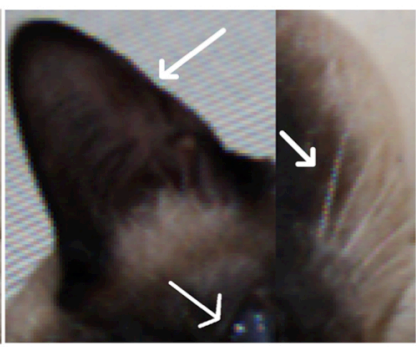

(c)

Figure 2.6 (a) An original scene. Inset at lower left: Bayer mosaic. (b) Close-ups of marked areas after high-quality (adaptive) and (c) after low-quality (non-adaptive) demosaicing. Artifacts shown here are zippering on the sides of the ear and false colors near the white pixels of the whiskers and the eye.

\subsubsection{RGB Equalization}

RGB equalization, often termed the "wrong von Kries model" (Westland and Ripamonti 2004), effectively ensures that the R,G and B values recorded for a gray calibration target are equal to each other. For a pixel $p$ in the $i^{\text {th }}$ color channel of a linear image, RGB equalization is performed as: 


$$
p_{i}^{\mathrm{WB}}=\frac{p_{i}-D S_{i}}{W S_{i}-D S_{i}} \quad \mathrm{i}=\mathrm{R}, \mathrm{G}, \mathrm{B}
$$

where $p_{i}^{\mathrm{WB}}$ is the intensity of the resulting white balanced pixel in the $i^{\text {th }}$ channel, and $D S_{i}$ and $W S_{i}$ are the values of the dark standard and the white standard in the $i^{t h}$ channel, respectively. The dark standard is usually the black patch in a calibration target, and the white standard is a gray patch with uniform reflectance spectrum (often, the white patch). A gray photographic target (Figure 2.7) is an approximation to a Lambertian surface (one that appears equally bright from any angle of view) and has a uniformly distributed reflectance spectrum. On such a surface the RGB values recorded by a camera are expected to be equal; but this is almost never the case due to a combination of camera sensor imperfections and spectral properties of the light field (Westland and Ripamonti 2004); RGB equalization compensates for that.

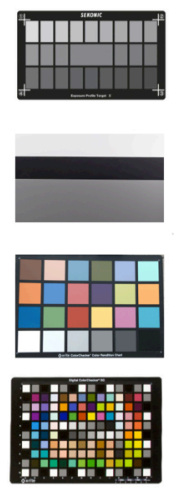

(a)

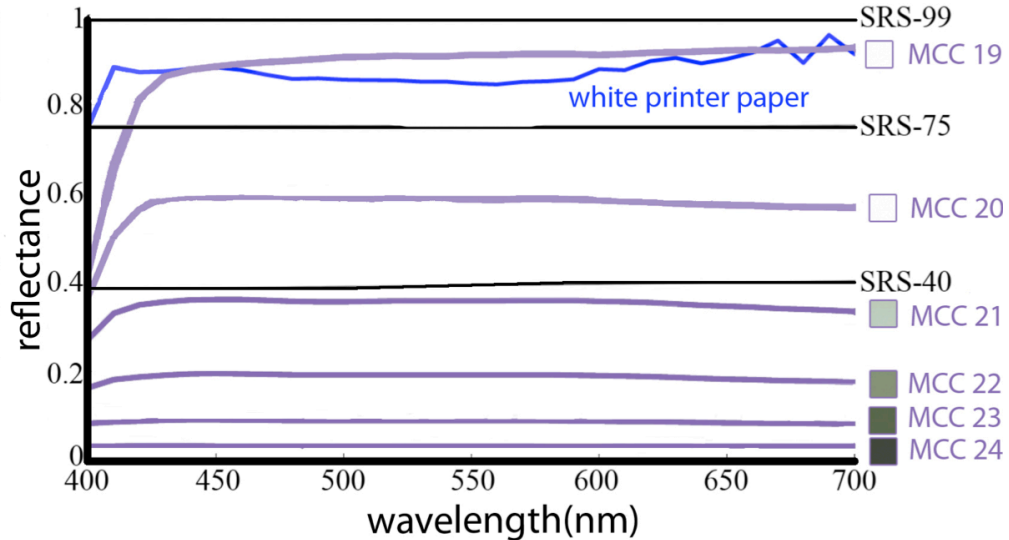

(b)

Figure 2.7 (a) Examples of photographic calibration targets. Top to bottom: Sekonik Exposure Profile Target II, Digital Kolor Kard, Macbeth ColorChecker (MCC), ColorChecker Digital. (b) Reflectance spectra (400-700nm) of SpectralonTM targets (black curves, prefixed with SRS-), gray patches of the MCC (purple), and a white sheet of printer paper (blue). Note that MCC 23 has a flatter spectrum than the white patch (MCC 19). The printer paper is bright and reflects most of the light, but it does not do so uniformly at each wavelength.

\subsection{Color transformation}

Two different cameras record different RGB values for the same scene due to differences in color sensitivity. This is true even for cameras of the same make and model (Stevens et al. 2007). Thus, the goal of applying a color transformation is to minimize this difference by converting device-specific colors to a standard, device-independent space (Figure 2.8). Such color transformations are constructed by imaging calibration targets. Standard calibration targets contain patches of colors that are carefully selected to provide a basis to the majority of natural reflectance spectra. A transformation matrix $\mathrm{T}$ between camera color space and a device-independent color space is computed as a linear least squares regression problem:

$$
V_{\text {ground truth }}^{\mathrm{XYZ}}=T V_{\text {linear }}^{\mathrm{RGB}}
$$


Here, $V_{\text {ground truth }}^{X Y Z}$ and $V_{\text {linear }}^{R G B}$ are $3 \times N$ matrices where $\mathrm{N}$ is the number of patches in the calibration target. The ground truth $\mathrm{XYZ}$ tri-stimulus values $V_{\text {ground truth }}^{X Y Z}$ can either be the published values specific to that chart, or they could be calculated from measured spectra (Ch. 2.7). The RGB values $V_{\text {linear }}^{R G B}$ are obtained from the linear RGB image of the calibration target. Note that the published XYZ values for color chart patches can be used only for the illuminants that were used to construct them (e.g., CIE illuminants D50 or D65); for other illuminants a white point conversion (Eq. 2.3\&2.4) should first be performed on linear RGB images.
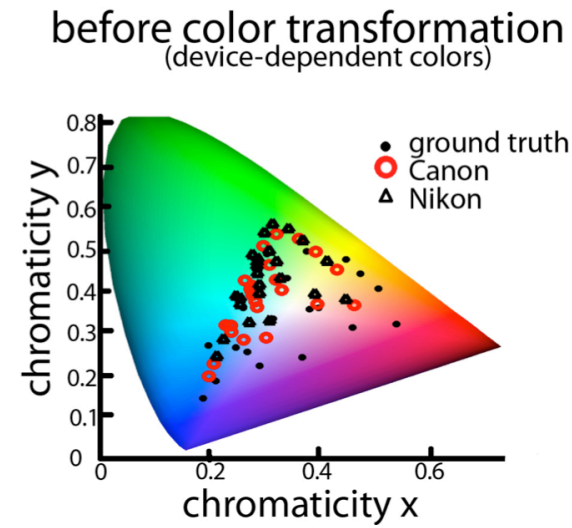

Figure 2.8 Chromaticity of Macbeth ColorChecker patches captured by two cameras, whose sensitivities are given in Fig. 5 in device-dependent and independent color spaces.

The $3 \times 3$ transformation matrix T (see (Westland and Ripamonti 2004) for other polynomial models) is then estimated from Eq. 2.6:

$$
T=V_{\text {ground truth }}^{X Y Z}\left[V_{\text {linear }}^{R G B}\right]^{+}
$$

where the superscript + denotes the Moore-Penrose pseudo-inverse of the matrix $V_{\text {linear }}^{R G B}$. This transformation $\mathrm{T}$ is then applied to a white-balanced novel image $\mathrm{I}_{\text {linear }}^{R G B}$ :

$$
I_{\text {corrected }}^{\mathrm{XYZ}}=T I_{\text {linear }}^{\mathrm{RGB}}
$$

to obtain the color-corrected image $I_{\text {corrected }}^{X Y Z}$, which is the linear, device-independent version of the raw camera output. The resulting image $I_{\text {corrected }}^{X Y Z}$ needs to be converted to RGB before it can be displayed on a monitor. There are many RGB spaces and one that can represent as many colors as possible should be preferred for computations (e.g., Adobe wide gamut) but when displayed, the image will eventually be shown within the boundaries of the monitor's gamut.

In Eq. 2.6, we did not specify the value of $\mathrm{N}$, the number of patches used to derive the matrix T. Commercially available color targets vary in the number of patches they have, ranging between tens and hundreds. In general, higher number of patches used does not guarantee an increase in color transformation accuracy. Alsam and Finlayson (Alsam 
and Finlayson 2008) found that 13 of the 24 patches of a Macbeth ColorChecker (MCC) are sufficient for most transformations. Intuitively, using patches whose radiance spectra span the subspace of those in the scene yields the most accurate transforms; we demonstrate this in Figure 2.10. Given a scene that consists of a photograph of a MCC taken under daylight, we derive $\mathrm{T}$ using an increasing number of patches (1-24 at a time) and compare the total color transformation error in each case. We use the same image of the $\mathrm{MCC}$ for training and testing because this simple case provides a lower bound on error. We quantify total error using:

$\mathrm{e}=\frac{1}{N} \sum_{i=1}^{N} \sqrt{\left(L_{i}-L_{G T}\right)^{2}+\left(A_{i}-A_{G T}\right)^{2}+\left(B_{i}-B_{G T}\right)^{2}}$

where an $L A B$ triplet is the representation of an XYZ triplet in the CIE LAB color space (which is perceptually uniform); $\mathrm{i}$ indicates each of the N patches in the MCC and GT is

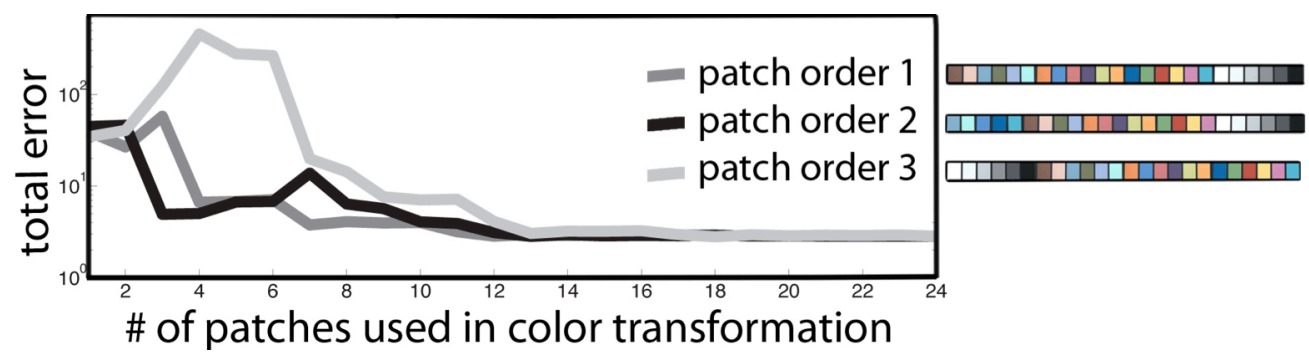

Figure 2.9 Using more patches for a color transformation does not guarantee increased transformation accuracy. In this example, color transformation error is computed after 1-24 patches are used. There were many possible ways the patches could have been selected, only three are shown here. Regardless of patch ordering, overall color transformation error is minimized after the inclusion of the 18th patch. First six patches of orders 1 and 2 are chromatic and for order 3 , they are achromatic. The errors associated with order 3 are higher initially because the scene, which consists of a photo of a Macbeth ColorChecker, is mostly chromatic. Note that it is not possible to have the total error be identically zero even in this simple example due to numerical error and noise.

the ground-truth value for the corresponding patch. Initially, the total error depends on the ordering of the color patches. Since it would not be possible to simulate $24 !(6.2045 \times$ 1023 ) different ways the patches could be ordered, we computed error for three cases (see Figure 2.9 legend). Initial error is the highest for patch order 3 because the first six patches of this ordering are the achromatic and this transformation does poorly for the MCC, which is composed of mostly chromatic patches. Patch orderings 1 and 2 on the other hand, start with chromatic patches and the corresponding initial errors are roughly an order of magnitude lower. Regardless of patch ordering, the total error is minimized after the inclusion of the 18th patch. 


\subsection{Scene-specific color calibration (SSCC)}

In Ch. 2.7, we outlined the process for building a $3 \times 3$ matrix $T$ that transforms colors from a camera color space to the standard CIE XYZ color space. It is apparent from this process that the calibration results are heavily dependent on the choice of the calibration target, or the specific patches used. Then, we can hypothesize that if we had a calibration target that contained all the colors found in a given scene, and only those colors, we would obtain a color

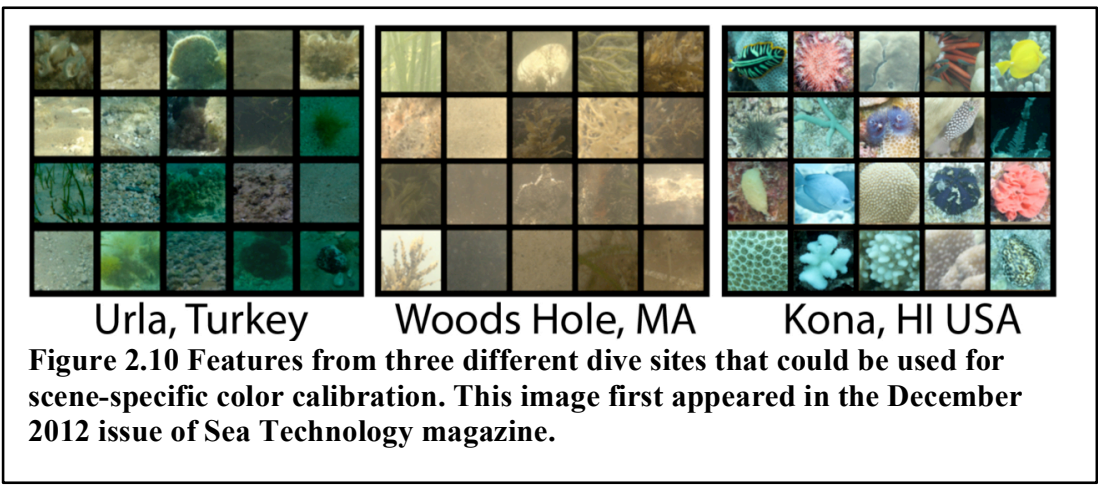
transformation with minimum error. In other words, if the colors used to derive the transformation $\mathrm{T}$ were also the colors used to evaluate calibration performance, the resulting error would be minimal - this is the goal of scene-specific color calibration.

The color signal that reaches the eye, or camera sensor, is the product of reflectance and irradiance (Figure 2.5), i.e. radiance (Eq. 2.1\&2.2). Therefore, how well a calibration target represents a scene depends on both the chromatic composition of the features in the scene (reflectance) and the ambient light profile (irradiance). For example, a scene viewed under daylight will appear monochromatic if it only contains different shades of a single hue, even though daylight is a broadband light source. Similarly, a scene consisting of an MCC will appear monochromatic when viewed under a narrowband light source even though the MCC patches contain many different hues.

Consumer cameras carry out color transformations from camera-dependent color spaces (i.e. raw image) to camera-independent color spaces assuming that a scene consists of reflectances similar to those in a standard color target, and that the ambient light is broadband (e.g., daylight or one of common indoor illuminants), because most scenes photographed by consumer cameras have these properties. We call scenes that can be represented by the patches of a standard calibration target ordinary. Non-ordinary scenes, on the other hand, have features whose reflectances are not spanned by calibration target patches (e.g. in a forest there may be many shades of greens and browns that common calibration targets do not represent), or are viewed under unusual lighting (e.g. under monochromatic light). In the context of scientific imaging non-ordinary scenes may be encountered often; we give examples in Ch 2.9.

For accurate capture of colors in a non-ordinary scene, a color calibration target specific to that scene is built. This is not a physical target that is placed in the scene as described in Ch. 2.7; instead, it is a matrix containing tri-stimulus values of features from that scene. Tri-stimulus values are obtained from the radiance spectra measured from features in the scene. In Figure 2.10 we show features from three different underwater habitats from which spectra, and in turn tri-stimulus values, can be obtained. Spectra are converted into tri-stimulus values as follows (Reinhard et al. 2008):

$$
X_{j}=\frac{1}{K} \sum_{i}^{n} \overline{x_{\jmath, l}} R_{i} E_{i}
$$


where $X_{1}=X, X_{2}=Y, X_{3}=Z$, and $K=\sum_{i}^{n} \bar{y} E_{i}$. Here, $i$ is the index of the wavelength steps at which data were recorded, $R_{i}$ is the reflectance spectrum and $E_{i}$ the spectrum of irradiance; $\overline{x_{\jmath, l}}$ are the values of the CIE 1931 color matching functions $x, y, z$ at the ith wavelength step, respectively.

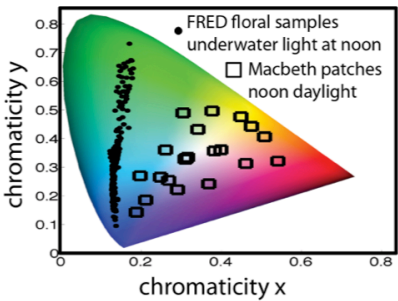

(a)

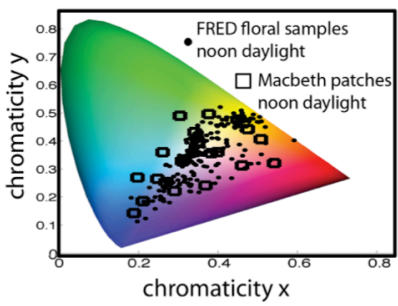

(c)

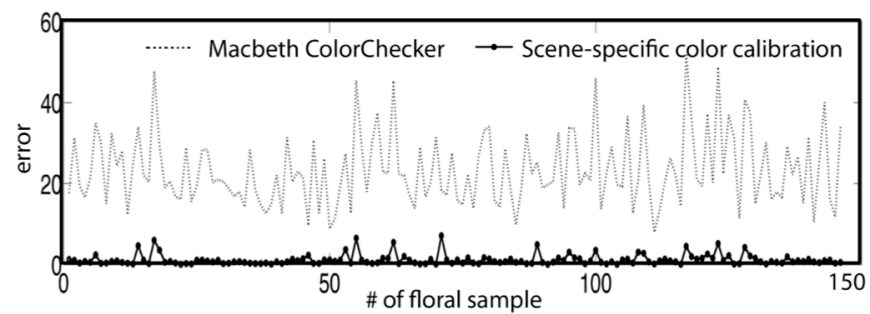

(b)

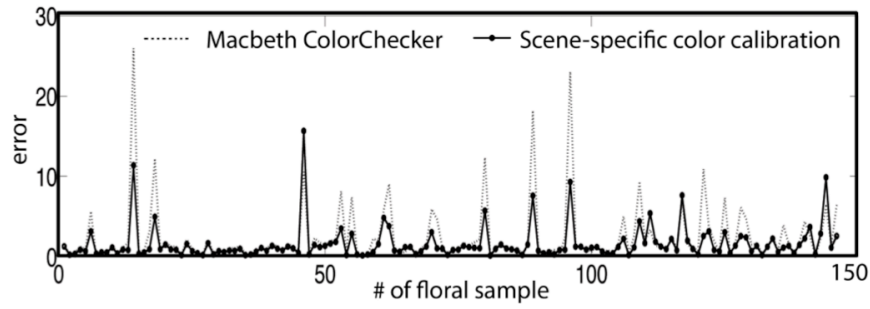

(d)

Figure 2.11 Scene-specific color transformation improves accuracy. (a) A "non-ordinary" scene that has no chromaticity overlap with the patches in the calibration target. (b) Mean error after scene-specific color calibration (SSCC) is significantly less than after using a calibration chart. (c) An "ordinary" scene in which MCC patches span the chromaticities in the scene. (d) Resulting error between the MCC and scene-specific color transformation is comparable, but on average, still less for SSCC.

Following the calculation of the XYZ tri-stimulus values, Eq. 2.6-2.8 can be used as described in Ch. 2.7 to perform color transformation. However, for every feature in a scene whose $\mathrm{XYZ}$ values are calculated, a corresponding RGB triplet that represents the camera color space is needed. These can be obtained in two ways: by photographing the features at the time of spectral data collection, or by simulating the RGB values using the spectral sensitivity curves of the camera (if they are known) and ambient light profile. Eq. 2.10 can be used to obtain the camera RGB values by substituting the camera spectral sensitivity curves instead of the color matching functions. In some cases, this approach is more practical than taking photographs of the scene features (e.g., under field conditions when light may be varying rapidly), however spectral sensitivity of camera sensors is proprietary and not made available by most manufacturers. Manual measurements can be done through the use of a monochromator (Nakamura 2005), a set of narrowband interference filters (Mauer and Wueller 2009), or empirically (Finlayson et al. 1998; Hong et al. 2001; Barnard and Funt 2002; Cheung et al. 2005; Jiang et al. 2013). 


\subsubsection{Scene-specific color calibration for non-ordinary scenes}

To create a non-ordinary scene, we used 292 natural reflectance spectra randomly selected from a floral reflectance database (Arnold et al. 2010), and simulated their radiance with the underwater light profile at noon shown in Figure 2.11a (scene 1). While this seems like an unlikely combination, it allows for the simulation of chromaticity coordinates (Figure 2.11a, black dots) that are vastly different than those corresponding to an MCC under noon daylight (Figure 2.11a, black squares), using naturally occurring light and reflectances. We randomly chose $50 \%$ of the floral samples to be in the training set for SSCC, and used the other $50 \%$ as a novel scene for testing. When this novel scene is transformed using an MCC, the mean error according to Eq. 2.9 was 23.8 and with SSCC, it was 1.56 (just noticeable difference threshold is 1). We repeated this transformation 100 times to ensure test and training sets were balanced and found that the mean error values remained similar. Note that the resulting low error with SSCC is not due to the high number of floral samples used (146) in training for SSCC, compared to only 24 patches in a MCC. Repeating this analysis with a training set of only 24 randomly selected floral samples did not change results significantly.

\subsubsection{Scene-specific color calibration for ordinary scenes}

We used the same spectra from scene 1 to build an ordinary scene (scene 2), i.e. a scene in which the radiance of the floral samples (Figure 2.11c, black dots) are spanned by the radiance of the patches of an MCC (Figure 2.11c, black squares). In this case, the average color transformation error using an MCC was reduced to 2.3; but it was higher than the error obtained using SSCC (Figure 2.11d), which was 1.73 when 24 patches were used for training, and 1.5 with 146 patches.

Table 2 Summary of post-processing steps for raw images

\begin{tabular}{|c|c|c|c|c|}
\hline \# & Camera, Light & Demosaic & White balance & Color transformation \\
\hline I & $\begin{array}{l}\text { Sony A700, Incandescent indoor } \\
\text { light }\end{array}$ & \multirow{5}{*}{$\begin{array}{l}\text { Adobe DNG } \\
\text { converter } \\
\text { Version 6.3.0.79 } \\
\text { (for list of other } \\
\text { raw image } \\
\text { decoders, see } \\
\text { http://www.cyber } \\
\text { com.net/ dcoffin/ } \\
\text { dcraw/) }\end{array}$} & \multirow{2}{*}{$\begin{array}{l}\text { 4th gray \& black in } \\
\text { MCC } \\
\text { Eq. } 5\end{array}$} & $\begin{array}{l}\text { None - analysis in the } \\
\text { camera color space }\end{array}$ \\
\hline II & $\begin{array}{l}\text { Canon EOS 1Ds Mark II, } \\
\text { Daylight }\end{array}$ & & & $\mathrm{MCC}$ and SSCC \\
\hline III & $\begin{array}{l}\text { Canon Rebel T2, Low-pressure } \\
\text { sodium light }\end{array}$ & & \multirow{2}{*}{$\begin{array}{l}\text { White point of } \\
\text { ambient light } \\
\text { spectrum } \\
\text { Eq. } 5 \& 10\end{array}$} & $\begin{array}{l}\text { None - analysis in the } \\
\text { camera color space }\end{array}$ \\
\hline IV & $\begin{array}{l}\text { Canon EOS 1Ds Mark II, } \\
\text { Daylight }\end{array}$ & & & $\mathrm{SSCC}$ \\
\hline V & $\begin{array}{l}\text { Canon EOS 5D Mark II, Daylight } \\
+2 \text { DS160 Ikelite strobes }\end{array}$ & & $\begin{array}{l}\text { 4th gray\& black in } \\
\text { MCC } \\
\text { Eq. } 5\end{array}$ & $\mathrm{MCC}$ \\
\hline
\end{tabular}




\subsection{Examples of the use of COTS cameras for scientific imaging}

Imaging and workflow details for the examples in this section are given in Table 2.

\section{Example I - Using colors from a photograph to quantify temperature distribution on a} surface (Figure 2.3 Steps: 1-3)

With careful

calibration, it is

possible to use

inexpensive cameras

to extract reliable

temperature readings

from surfaces painted

with temperature-

sensitive dyes, whose

emission spectrum

(color) changes when

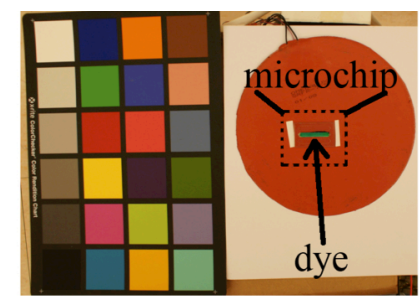

(a)

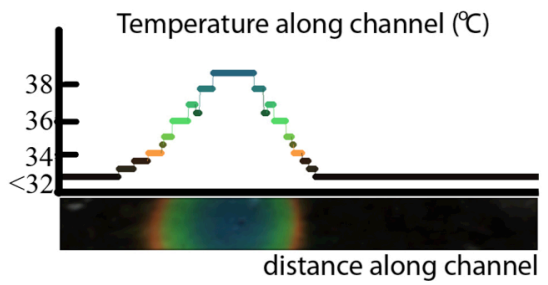

(b)

Figure 2.12 Temperature distribution along the microchip channel which is locally heated to $39^{\circ} \mathrm{C}$ (colored region) while the rest was kept below $32^{\circ} \mathrm{C}$ (black region).

heated or cooled.

Gürkan et al. (Gurkan et al. 2012) stained the channels in a microchip with thermosensitive dye (Edmund Scientific, Tonawanda, NY; range $32^{\circ} \mathrm{C}-41^{\circ} \mathrm{C}$ ) and locally heated it one degree at a time using thermo-electric modules. At each temperature step a set of baseline raw (linear RGB) photographs were taken. Then, novel photographs of the chip (also in raw format) were taken for various heating and cooling scenarios. Each novel photograph was white balanced using the calibration target in the scene (Figure 2.12). Since all images being compared were acquired with the same camera, colors were kept in the camera color space and no color transformation was applied. To get the temperature readings, colors along the microchip channel were compared to the baseline RGB values using the $\Delta E_{2000}$ metric (Luo et al. 2001) and were assigned the temperature of the nearest baseline color.

Example II: Use of inexpensive COTS cameras for accurate artwork photography (Figure 2.3 Steps 1-4)

Here, we quantify the error associated with using a standard calibration target (vs. SSCC) for imaging an oil painting (Figure 2.13). Low-cost, easy-to-use consumer cameras and standard color calibration targets are often used in artwork archival; a complex and specialized application to which many fine art and cultural heritage institutions allocate considerable resources. Though many museums in the Unites States have been using digital photography for direct capture of their artwork since the late 1990s (Rosen and Frey 2005), Frey and colleagues (Frey and Farnand 2011) found that some institutions did not include color calibration targets in their imaging workflows at all. For this example, radiance from 36 points across the painting were measured, and it was found that their corresponding tri-stimulus values were within the span of the subspace of the MCC patches under identical light conditions, i.e. an ordinary scene. The MCC-based color transformation yielded an average $\Delta E_{2000}$ value of 0.21 , and 0.22 for SSCC, both below the just noticeable difference threshold of 1 . However, the average error was 31.77 for the jpg output produced by the camera, used in auto setting. For this ordinary scene, there was no advantage to be gained from SSCC and the use of a standard calibration 
target with the workflow in Figure 2.3 significantly improved color accuracy over the incamera processed image.

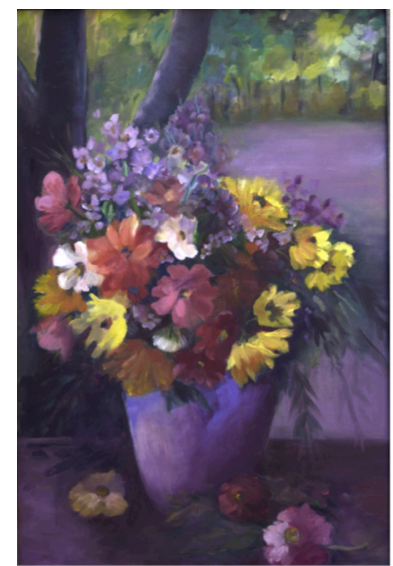

(a)

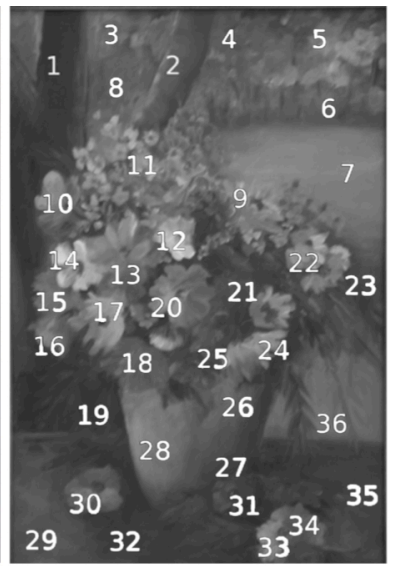

(b)

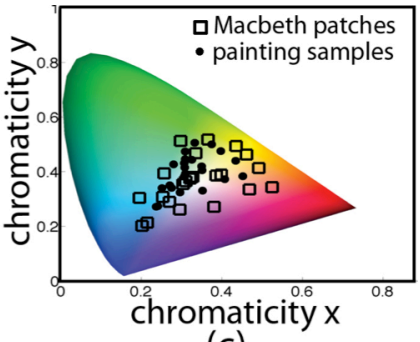

(c)

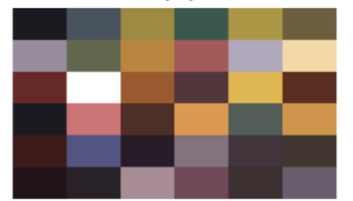

(d)

Figure 2.13 Example II: Use of inexpensive COTS cameras for accurate artwork photography. (a) An oil painting under daylight illumination. (b) Thirty-six points from which ground truth spectra were measured. (b) Chromatic loci of the ground truth samples compared to Macbeth ColorChecker patches, under identical illumination. (d) sRGB representation of the colors used for scene-specific calibration. Artwork: Fulya Akkaynak.

Example III - Capturing photographs under monochromatic low pressure sodium light (Figure 2.3 Steps 1-3)

A monochromatic light spectrum $\mathrm{E}$ can be approximated by an impulse centered at the peak wavelength $\lambda_{p}$ as $E=C \cdot \delta\left(\lambda-\lambda_{p}\right)$ where $\mathrm{C}$ is the magnitude of intensity, and $\delta$ is the Dirac delta function whose value is zero everywhere except when $\lambda=\lambda_{p}$. When $\lambda=\lambda_{p}, \delta=1$. It is not trivial to capture monochromatic scenes using COTS cameras accurately, because they are optimized for use with indoor and outdoor broadband light sources. We used low-pressure sodium light $\left(\lambda_{p}=589 \mathrm{~nm}\right)$ to investigate the effect of color (or lack of) on visual perception of the material properties of objects. Any surface viewed under this light source appears as a shade of orange because $x_{3}=z=0$ at $\lambda=589 \mathrm{~nm}$ for the CIE XYZ human color matching functions (also for most consumer cameras), and in turn, $X_{3}=Z=0$ at $\lambda=589 \mathrm{~nm}$ (Eq. 2.10). Subjects were asked to view real textiles once under sodium light and once under broadband light and answer questions about their material properties. The experiment was then repeated using photographs of the same textiles. To capture the appearance of colors under sodium light accurately, its irradiance was recorded using a spectrometer fitted with an irradiance probe. Then, the tri-stimulus values corresponding to its white point were calculated and used for RGB-equalization of linear novel images. Due to the monochromatic nature of the light source, there was no need to also apply a color transformation; adjusting the white point of the illuminant in the images ensured that the single hue in the scene was mapped correctly. The white point of the illuminant was indeed captured incorrectly when the camera was operated in auto mode, and the appearance of the textiles was noticeably different (Figure 2.14). 


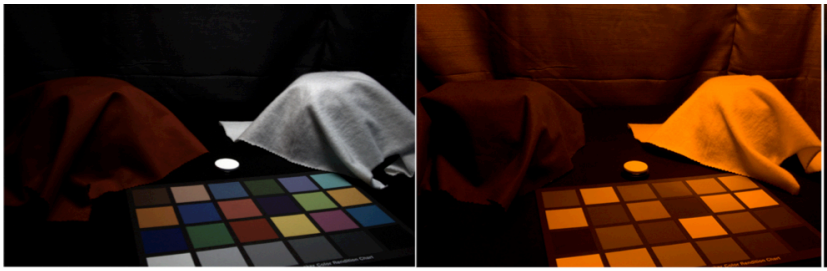

(a) (b)

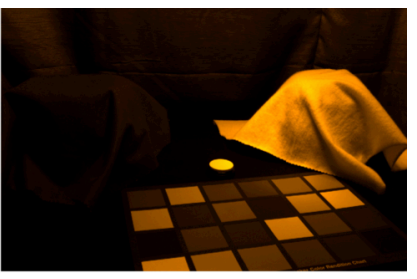

(c)

Figure 2.14 Example III Capturing photographs under monochromatic low-pressure sodium light. (a) A pair of fabrics under broadband light. (b) A jpg image taken with the auto settings of a camera, under monochromatic sodium light. (c) Image processed using scene-specific color calibration according to the flow in Figure 2.3.

\section{Example IV: In situ capture of a camouflaged animal and its habitat underwater (Figure} 2.3 Steps 1-4)

Imaging underwater is challenging because light is fundamentally different from what we encounter on land (Figure 2.5) and each underwater habitat is different from another in terms of the colorfulness of substrates (Figure 2.10). Thus, there is no global color chart that can be used across underwater environments, making underwater scenes (except for those very close to the surface) non-ordinary scenes. The majority of underwater imaging falls into three categories motivated by different requirements: (1) using natural light to capture a scene exactly the way it appears at a

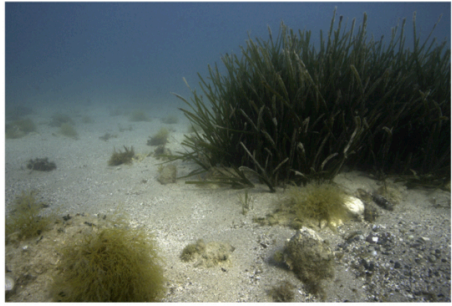

(a)

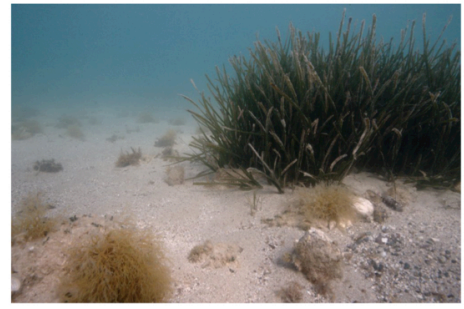

(c)

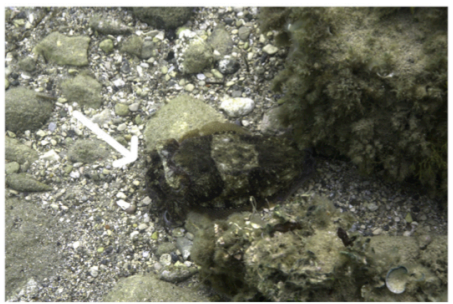

(b)

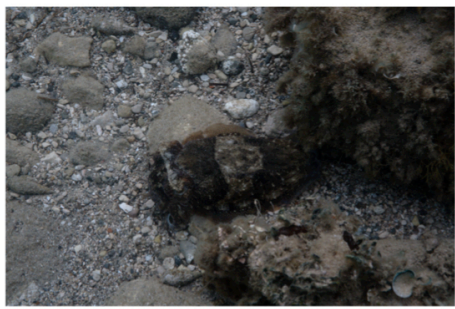

(d)

Figure 2.15 Example IV In situ capture of (a) an underwater habitat and (b) a camouflaged cuttlefish using scene-specific color calibration with features similar to those shown in Fig. 10 for Urla, Turkey. (c) and (d) are jpg outputs directly from the camera operated in auto mode and have a visible red tint as a consequence of in-camera processing.

given depth (2) using

natural light to capture an underwater scene but post-processing to obtain its appearance on land and (3) introducing artificial broadband light, (e.g., (Vasilescu 2009), in situ to capture the scene as it would have appeared on land (Example V). Here we give an example for case (1): capture of the colors of the skin of a camouflaged animal exactly the way an observer in situ would have seen them. For this application, we surveyed a dive site and built a database of substrate reflectance and light spectra (Akkaynak et al. 2013). Novel (raw) images taken at the same site were white balanced to match the white point of the ambient light. We then used the spectral database built for this dive site for SSCC (Figure 2.15). The green hue in the resulting images may appear unusual for two 
reasons. First, a light-adapted human diver may not have perceived that green color in situ due to color constancy and therefore may not remember the scene to appear that way. Second, most professional underwater photographs we are familiar with are either postprocessed to appear less green (case 2), or they are taken with strobes that introduce broadband light to the scene to cancel the green appearance before image capture (case 3). This kind of processing is indeed similar to the processing that may be performed by a COTS camera; the camera will assume a common land light profile (unless it is being operated in a pre-programmed underwater mode), ignorant of the long-wavelength attenuated ambient light, and this will result in boosting of the red channel pixel values.

Example V: Consistent underwater color correction across multiple images (Figure 2.3

Steps: $1-4)$

Repeated consistent measurements are the foundation of ecological monitoring projects. In the case of corals, color information can help distinguish between different algal functional groups, or even establish coral health change

(Siebeck et al. 2006). Until now, several color correction targets were used for coral monitoring

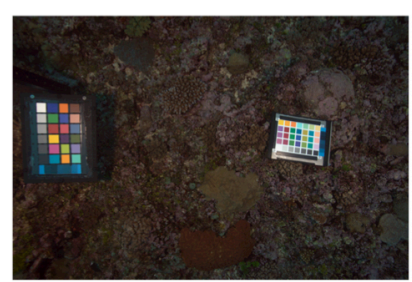

(a)

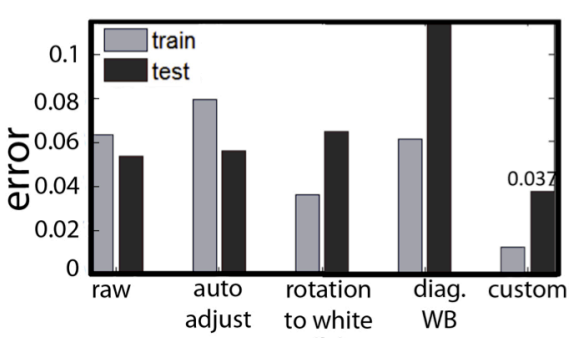

(b)

Figure 2.16 Example V Consistent underwater color correction. (a) Top row: six of the 68 raw images taken in clear water in Totoya reef, Fiji, before color correction; bottom row: after. In each frame, the color chart on the left was used for calibration, and the one on the right for testing. (b) Average error for several color correction method for the calibration (blue) and the test (red) chart. Our method achieves the lowest error and is the only method to improve over the raw images of the test chart.

(Winters et al. 2009), but

not tested for consistency. To test our method's performance for consistent color capture, we attached one color chart to the camera using a monopod, and placed another one manually in different locations in the field of view (Fig. 17). We tested several correction algorithms: automatic adjustment, white balancing and the algorithm presented in this paper (Fig. 3) and defined the error as consistency of the corrected color. For each of the $\mathrm{M}=35$ patches in the $\mathrm{N}=68$ corrected images, we calculated mean chromaticity as: $\overline{r_{m}}=\frac{1}{N} \sum_{n=1}^{N} r_{m, n}, \overline{g_{m}}=\frac{1}{N} \sum_{n=1}^{N} g_{m, n}, m=1 \ldots M$ where $r=R /(R+G+B), g=$ $G /(R+G+B)$ and defined the patch error to be $E_{m}=\frac{1}{N} \sum_{i=1}^{N}\left\|\left(r_{m, i}, g_{m, i}\right)-\left(\overline{r_{m}}, \overline{g_{m}}\right)\right\|$ with total error as the average across all $\mathrm{M}$ patches. Our algorithm yielded the most consistent colors, opening the possibility for the use of color targets designed for land photography in marine ecology images. 


\subsection{Conclusion}

Concepts discussed in this work are central topics in research fields such as applied optics, computational photography, optical imaging and colorimetry. While professional photographers and researchers in fields like computer vision frequently use raw images and calibration targets to transform device-dependent colors to a deviceindependent space, the importance of manually calibrating consumer camera images has only recently become understood by animal biology scholars. We streamlined these calibration steps in a simple and direct manner to make them accessible to researchers from all areas, without assuming familiarity with imaging-related sciences or the technology behind consumer cameras.

Four steps are necessary for the use of consumer cameras as consistent and accurate data acquisition instruments: raw image capture, demosaicing of the raw image, white balancing and color transformation (Figure 2.3). How each step of processing should be executed depends on the particular application; we have given examples in Chapter 2.9. When pixel intensity values are to be used as scientific data, at the very least an off-the-shelf calibration target should used to obtain consistent colors to allow for reproducibility of work by other researchers with no knowledge of the particular imaging instrument. If accurate color capture is also desired in addition to consistency, reflectance and irradiance spectra (the product of which yields radiance) collected from features from the scene can be used to obtain tristimulus values, forming the baseline for a scenespecific calibration target. A scene-specific approach to color transformation is especially important when the scene contains objects whose chromaticities are vastly different from those in an average scene we encounter on land, or if it was viewed under a narrowbroadband light, or both. In the case of a simulated underwater scene, we showed that color transform could be achieved with approximately a 20-fold reduction in error with scene-specific color calibration.

We recognize that the methodology described here adds some complication to the very feature that makes COTS cameras popular - ease of use. To allow for easier adoption of the extra steps required and their seamless integration into research projects, we provide a toolbox of functions written in MATLAB programming language (Mathworks, Inc. Natick MA) available for download at the following link: http://www.mathworks.com/matlabcentral/fileexchange/42548 . For some steps in our workflow, knowing the spectral sensitivities of a specific camera is advantageous. Camera manufacturers do not provide these curves and the burden falls on the users to derive them. Carrying out a survey to collect spectral data from a site to perform scenespecific color calibration requires the use of a spectroscopic imaging device, which adds to the overall cost of the study and may extend its duration. Yet, despite the extra effort required to calibrate them for scientific purposes, COTS cameras can have more utility than hyper-spectral imagers or spectrometers depending for applications requiring image capture in the visible range. 


\section{Chapter 3}

\section{Unbiased data acquisition: Spectrometers ${ }^{2}$}

\subsection{Introduction}

In the field of animal coloration and camouflage, spectrometers with optical fibers are frequently used to measure colors from an animal's habitat, nest, eggs, body parts, skin, fur and plumage (Table 3). Spectra measured from these features are then used in models that quantify color discriminability according to human or non-human visual systems. In terms of color capture, spectrometers have a clear advantage over consumer cameras (see Chapter 2) because they densely sample the electromagnetic spectrum, typically imaging between 70-100 bands, but often a thousand or more. In addition, they image outside the visible range, which most consumer cameras cannot. However, spectrometers collect data in a point-by-point fashion which means that they are not suitable for recording colors from textured surfaces, i.e. those with high frequency color or pattern elements. Even in the case of surfaces with solid colors and a carefully gridded, sequential data collection setup, the distance of the optical fiber to the sample, its diameter, numerical aperture and the measurement angle could affect the purity of the color signal recorded. If a spectral measurement is contaminated with neighboring colors, our assessment of the discriminability of that spectrum from another will no doubt be inaccurate. Given an optical fiber of known diameter and numerical aperture, and a body pattern feature of certain size, how can we quantify the degree of spectral contamination of our measurements?

In general, it is known to researchers that measurements should be taken as close to the surface being measured as possible. For laboratory measurements, optical fibers are fixed inside probe holders that have machined 45 and 90 -degree slots, securing the fiber at a distance that is nearly touching the sample at the desired angle. For spectroscopic measurements in the field, this is very difficult to establish; recording spectra closely from live animals is very challenging (Figure 3.1). In addition, ambient light conditions may change before a measurement is completed. The current standard for researchers conducting spectroscopic studies in the field is to report an estimate of the distance and angle of the optical fiber in their publications (Table 3). Inadvertently, not being able to get close enough to the animals, and then having to estimate the measurement distance results in errors regarding discriminability of colors for camouflage assessment. In this chapter, I develop an equation using geometrical optics that quantifies what these errors may be in the context of animal coloration. This equation depends only on the diameter and the numerical aperture of the optical fiber. Using the visual systems of a dichromatic ferret (Mustela putorious furo) and a trichromatic frog (Dendrobates pumilio), I test this equation on a pair of colors: (1) that have little spectral overlap simulating a conspicuous appearance, and (2) that have high spectral overlap simulating a camouflage pattern. Modeling a visual system that has one more photoreceptor than the di-chromat will help investigate how potential errors contaminated spectra cause translate into different assessments of color discriminability.

\footnotetext{
${ }^{2}$ Parts of this chapter was previously published. See (Akkaynak 2014) for full reference.
} 


\subsection{Background and related work}

One aspect of color vision is the ability to distinguish surfaces based on their spectral properties (Brainard 2001). In the field of animal biology, understanding animal color vision helps answer important scientific questions. For example, Maan and Cummings (Maan and Cummings 2008) found that for female poison frogs, the male's dorsal color is the only factor determining their choice of a mate. In addition to sexual selection, animals use color vision for behavioral tasks including finding food, recognizing objects and communicating with members of the same species (Kelber et al. 2003). To accurately capture and interpret how color signals are received, we must have a model of the observer animal's visual system. Analyzing color signals from the point-ofview of humans and making inferences about their appearances to animals often produces erroneous conclusions. For example Stoddard and Stevens (Stoddard and Stevens 2011) showed that some common cuckoo eggs (which are laid in nests of other species and often hatched and raised by unsuspecting host birds) appeared to have a good color match to the host eggs when viewed by humans, but had clear and quantifiable differences when modeled in avian color space. A detailed summary of how color signals can be modeled from the perspective of animals can be found in (Endler and Mielke Jr 2005). In this work, my focus is not on these models, but on the measurement of the color signals. Color is inherently difficult to measure objectively, because it is a sensation defined relative to the human visual system (Wyszecki and Stiles 2000). Visual systems of most other animals are different from our, and analyses from the perspective of other systems must start with unfiltered spectral data, encompassing the segment of the electromagnetic spectrum to which the animal is sensitive (see Chapter 2). Spectral information in a scene can most comprehensively be captured using a hyperspectral imager (Akkaynak et al. 2014), which, for each pixel in its sensor, records a densely sampled portion of the electromagnetic spectrum. Despite providing spectral data for every pixel in an image, hyperspectral imagers are not commonly used in studies of animal coloration (or most other research fields). This is because they are still expensive, physically bulky, have low resolution, create large digital files and are not fast enough for imaging moving objects (i.e. animals in the wild). Although consumer digital cameras are inexpensive, fast, compact and widely available, they cannot serve as substitutes for hyperspectral imagers because they record spectral information with limited spectral resolution (Akkaynak et al. 2014). Thus, spectrometers, which are effectively single-pixel hyperspectral imagers, provide a compact and affordable solution for animal coloration studies; some examples of recent studies measuring animal or habitat spectra and then modeling discriminability in the eyes of animal visual systems are given in Table 3.

Most spectroscopic studies are conducted in laboratory settings using carefully designed optical benches. Key elements of these setups are fiber holders, which are essentially guides that ensure spectra from the sample are measured as close to its surface as possible without making contact. For free-hand spectroscopic measurements, how close the optical fiber should be held at, and at what angle, for a feature of known size has not previously been quantified; that is described next. 
Table 3 Recent publications that assess the discriminability of colors relative based on measurements taken by spectrometers (partial list).

\begin{tabular}{|c|c|c|c|}
\hline Publication & $\begin{array}{l}\text { Fiber Distance \& } \\
\text { Angle }\end{array}$ & Spectra Measured & $\begin{array}{l}\text { Visual } \\
\text { System } \\
\text { Modeled }\end{array}$ \\
\hline (Chiao et al. 2011) & $\begin{array}{l}\text { Hyperspectral } \\
\text { N/A }\end{array}$ & European cuttlefish & Fish \\
\hline (Isaac and Gregory 2013) & $\begin{array}{l}\text { Spectrometer } \\
100 \mu \mathrm{m} \\
2 \mathrm{~mm} / 90^{\circ}\end{array}$ & $\begin{array}{l}\text { Western terrestrial } \\
\text { garter snake }\end{array}$ & $\begin{array}{l}\text { Ferret, } \\
\text { human, birds }\end{array}$ \\
\hline (Akkaynak et al. 2013) & $\begin{array}{l}\text { Spectrometer } \\
50 \text { and } 100 \mu \mathrm{m} \\
<3 \mathrm{~cm}\end{array}$ & European cuttlefish & Fish, human \\
\hline (Hanlon et al. 2013) & $\begin{array}{l}\text { Spectrometer } \\
400 \mu \mathrm{m} \\
2 \mathrm{~cm}\end{array}$ & $\begin{array}{l}\text { Giant Australian } \\
\text { cuttlefish }\end{array}$ & Fish \\
\hline (Finkbeiner et al. 2012) & $\begin{array}{l}\text { Spectrometer } \\
400 \mu \mathrm{m} \\
45^{\circ}\end{array}$ & $\begin{array}{l}\text { Passion-vine } \\
\text { butterflies }\end{array}$ & Birds \\
\hline (Lind et al. 2013) & $\begin{array}{l}\text { Spectrometer } \\
1000 \mu \mathrm{m} \\
45^{\circ}\end{array}$ & Blue tit & Birds \\
\hline (Maan and Cummings 2012) & $\begin{array}{l}\text { Spectrometer } \\
400 \mu \mathrm{m} \\
3 \mathrm{~mm} / 90^{\circ}\end{array}$ & $\begin{array}{l}\text { Strawberry, poison } \\
\text { frog }\end{array}$ & $\begin{array}{l}\text { Birds, crabs, } \\
\text { snakes, frogs }\end{array}$ \\
\hline (Bybee et al. 2012) & $\begin{array}{l}\text { Spectrometer } \\
400 \mu \mathrm{m} \\
45^{\circ}\end{array}$ & Heliconius butterfly & $\begin{array}{l}\text { Birds, } \\
\text { butterflies }\end{array}$ \\
\hline (Cortesi and Cheney 2010) & $\begin{array}{l}\text { Spectrometer } \\
200 \mu \mathrm{m} \\
45^{\circ}\end{array}$ & Marine opisthobranchs & Fish \\
\hline (Langmore et al. 2009) & $\begin{array}{l}\text { Spectrometer } \\
\text { N/A } \\
45^{\circ} \\
\end{array}$ & Cuckoo eggshell & Birds \\
\hline (Baldwin and Johnsen 2012) & $\begin{array}{l}\text { Spectrometer } \\
400 \mu \mathrm{m} \\
45^{\circ}\end{array}$ & Blue crab & Blue crab \\
\hline (Nokelainen et al. 2012) & $\begin{array}{l}\text { Spectrometer } \\
\text { N/A } \\
45-90^{\circ}\end{array}$ & Wood tiger moth & Blue tit \\
\hline (Stoddard et al. 2011) & $\begin{array}{l}\text { Spectrometer } \\
\text { N/A } \\
45^{\circ}\end{array}$ & Cuckoo eggs & Birds \\
\hline (Siddiqi et al. 2004) & $\begin{array}{l}\text { Spectrometer } \\
\text { N/A } \\
\text { N/A }\end{array}$ & $\begin{array}{l}\text { Strawberry poison } \\
\text { frogs }\end{array}$ & $\begin{array}{l}\text { Strawberry } \\
\text { poison frogs }\end{array}$ \\
\hline
\end{tabular}




\subsection{Finding optimal measurement distance and angle}

An optical fiber is a waveguide that propagates rays through total internal reflection (Bass et al. 2009). It is a cylindrically symmetric cable with a core that has high refractive index, $n_{\text {core }}$, surrounded by cladding with low refractive index, $n_{\text {clad }}$ (Figure 3.2). Fibers are characterized by their numerical aperture $(N A)$, which is defined relative to the refractive index of the medium of operation $\left(n_{\text {med }}\right)$ and the acceptance angle $\left(\theta_{0}\right)$

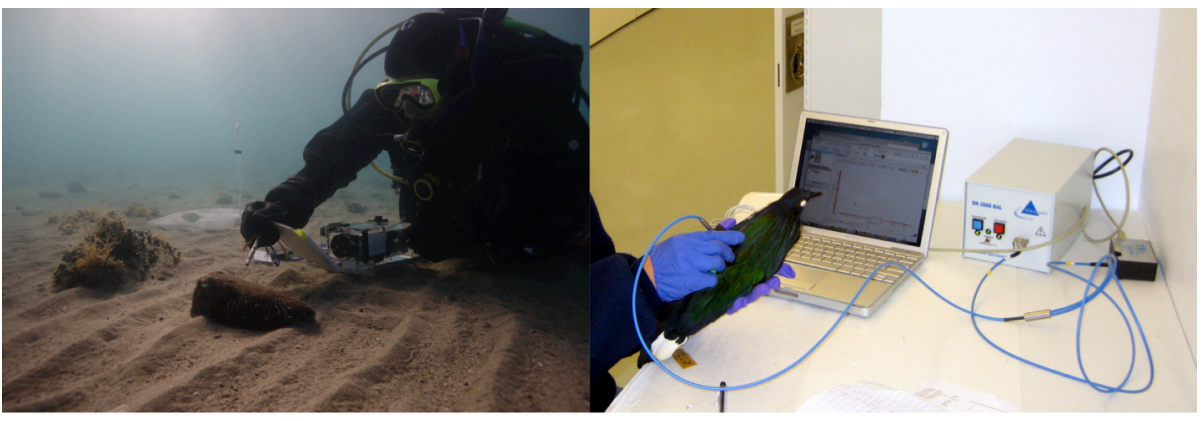

(a)

(b)

Figure 3.1 (a) A SCUBA diver extending the optical fiber attached to a spectrometer (in water and pressure proof housing) to record the spectrum of light reflected from the skin of a cuttlefish, in Urla, Turkey. Getting the optical fiber close enough to take accurate measurements from freely behaving animals in the wild is challenging. Photo credit: Derya Akkaynak. (b) Spectral measurements of a specimen can be recorded in a laboratory by getting the fiber as close to the specimen as possible without touching it. Photo courtesy of M.C. Stoddard \& K. Zyskowski, taken at the Ornithology Collections of the Peabody Museum of Natural History, Yale University, New Haven CT.

as:

$$
N A=n_{\text {med }} \cdot \sin \theta_{0}
$$

Acceptance angle is the largest incidence angle for incoming rays to undergo total internal reflection. The locus of all such rays is the cone of acceptance (shown in light gray in Figure 3.2). For most animal biology applications, the optical fiber is used in air $\left(n_{\text {med }} \approx 1\right)$, or water $\left(n_{\text {med }} \approx 1.33\right.$ ); the difference between fresh and salt water is usually ignored for practical purposes.

When the fiber is oriented along the Z-direction and is held at distance of $d$ from a surface tilted at an angle $\alpha$ relative to the XY plane, the cross-section of the acceptance cone on the surface is an ellipse (Figure 3.2). In the trivial case when $\alpha=0$, the plane will be parallel to the base of the cone, and intersection of the cone and the plane will be a circle. In the general case (derivation is given in Appendix), for a fiber with diameter $d_{f}$ and NA, the elliptical cross section is in the form

$$
\frac{\left(x-x_{c}\right)^{2}}{a^{2}}+\frac{\left(y-y_{c}\right)^{2}}{b^{2}}=1
$$

where

$$
\left(x_{c}, y_{c}\right)=\left(0, \frac{H_{S} \tan \alpha}{1 / t^{2}-(\tan \alpha)^{2}}\right)
$$


are the coordinates of the center of the ellipse, and

$$
a^{2}=\frac{H_{S}^{2} t^{2}}{\left(\frac{1}{t^{2}}-(\tan \alpha)^{2}\right)^{2}}
$$

and

$$
b^{2}=\frac{a^{2}}{\frac{1}{t^{2}}-(\tan \alpha)^{2}}
$$

are the squares of the semi-major and semi-minor axes, respectively. Here, it can be shown from geometry that $t=\frac{R}{H}=\tan \left(\sin ^{-1}\left(N A / n_{\text {med }}\right)\right)$. The height $H_{S}$ is measured from the apex of the cone to the intersection of the surface and the axis of the optical fiber (Figure 3.2) and is given by:

$$
H_{S}=d+\frac{d_{f}}{2 \tan \left(\sin ^{-1}\left(N A / n_{m e d}\right)\right)}
$$

\subsection{Application to animal color discrimination}

In this section, the effect of measurement distance and angle on the quality of the recorded spectral signals is investigated using the visual systems of a dichromatic ferret (Mustela putorious furo) and a trichromatic frog (Dendrobates pumilio). Fibers are assumed to have diameters 100, 400 and $1000 \mu \mathrm{m}$ based on those commonly used (Table 3 ), with $N A=0.22$. The operation medium is air $\left(n_{\text {med }}=1\right)$ and the illuminant is CIE D65,

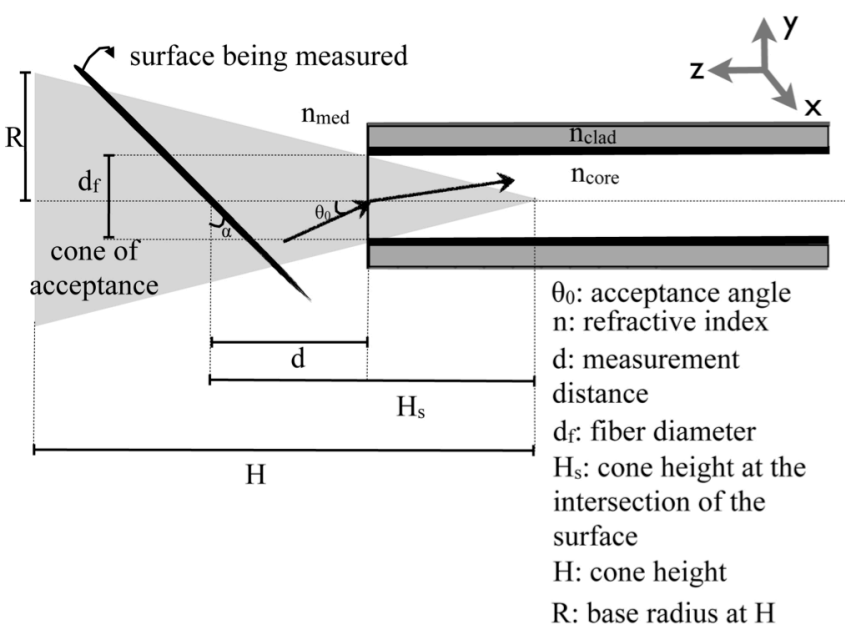

Figure 3.2 Field of view of an optical fiber. which is a theoretical light spectrum that approximates noon daylight. Solid color patches of dimensions $4.1 \mathrm{~cm} \times$ $4.1 \mathrm{~cm}$ (the actual size of patches on a Macbeth ColorChecker, Xrite, Inc.) on a synthetic test target are used to simulate two test cases relevant to animal coloration: (1) colors expected to yield high contrast (i.e. a conspicuous animal) and (2) colors expected to yield low contrast (i.e. a camouflaged animal). 


\subsubsection{Synthetic test stimuli}

A synthetic multi-spectral test target was created to show the effect of sampling colors erroneously. The target was designed to have the same arrangement and patch size as a Macbeth ColorChecker, a standard in color calibration. Since Macbeth charts only provide tristimulus values, complete spectra from the METACOW project (Fairchild and Johnson 2004) were substituted for matching colors. The spectra were obtained by averaging the reflectance spectra of a $50 \times 50$ pixel region from the left half of each METACOW sample. The resulting stimulus is a hyperspectral calibration target with square patches of solid colors, each of which has reflectance spectra in the interval 400$700 \mathrm{~nm}$ in $5 \mathrm{~nm}$ steps (Figure 3.3).

For case (1), spectra from patches A and B (Figure 3.3) were chosen to represent a conspicuous animal's pattern. The red and blue patches are expected to contrast highly for any visual system because they have little spectral overlap. To create a camouflaged animal that perfectly matches the colors of its background for case (2), the reflectance spectrum of patch A was copied onto the location of patch B, creating two neighboring patches that are identical. These patches would be expected to have minimal color contrast if the color was sampled without contamination from neighboring colors. Note that in both cases, there is black border between color patches (similar to the original Macbeth ColorChecker), which can affect the quality of the recorded signal.

\subsubsection{Mathematical similarity of two spectra}

It is often useful to assess the similarity (or, dissimilarity) of two spectra without referencing a biological visual system (Akkaynak et al. 2013). In this paper, the mathematical dissimilarity between the pure and recorded version of a spectrum is used as an objective measure of contamination, which is quantified using the Spectral Angle Mapper (SAM) metric (Yuhas et al. 1992):

$$
\theta_{S A M}=\cos ^{-1} \frac{S_{A}^{T} S_{B}}{\left\|S_{A}\right\|\left\|S_{B}\right\|}
$$

Here, spectra are treated as two high dimensional vectors $\left(S_{A}, S_{B}\right)$. The angle between the vectors can be thought of a measure of alignment; the smaller the angle, the more similar the two spectra are in shape. This metric is insensitive to differences in magnitude.

\subsubsection{Modeling of color discriminability}

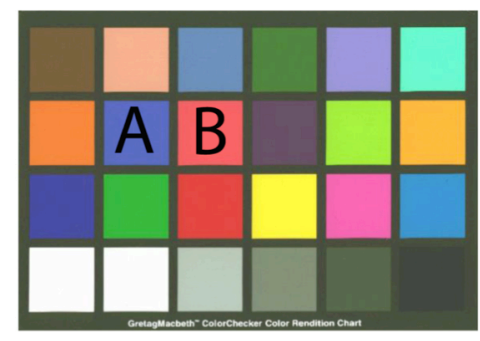

Macbeth ColorChecker ${ }^{\mathrm{TM}}$

(a)

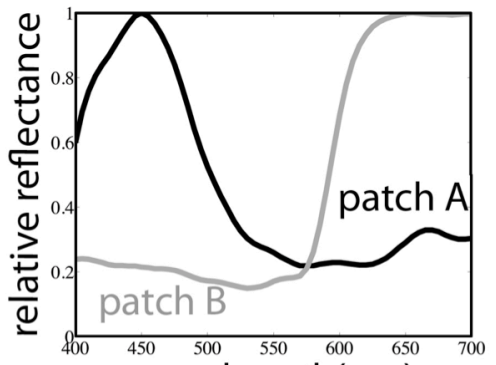

wavelength(nm)

(b)

Figure 3.3 Test stimuli used for assessment of color discrimination for a conspicuous animal (case 1), and a camouflaged animal (case 2). (a). The synthetic hyperspectral test target has the same layout as the Macbeth ColorChecker shown here. Patches $A$ and $B$ are those selected to be highly contrasting with each other for case (1). For case (2), the reflectance of patch $A$ is copied to the location of patch $B$, creating two patches with identical spectra next to each other, separated by a black border. (b) Reflectance spectra of patches A and B. 
One of the methods frequently used to assess discriminability of colors in animal vision is the receptor noise model developed by Vorobyev and Osorio (Vorobyev and Osorio 1998). In the absence of perceptual thresholds for the visual systems of most animals, this model predicts color discriminability by assuming that thresholds are set by color opponency mechanisms whose performance is limited by receptor noise. For a visual system with $n$ receptor channels, color is encoded with $n-1$ opponent mechanisms; the achromatic signal is disregarded and the relative proportion of each receptor determines the receptor noise. Similar to the CIE LAB-based distance metrics, two color stimuli are indistinguishable if the distance between them is one just noticeable difference (JND) or less. According to this model, color contrast $(\Delta S)$ for a dichromat visual system is computed as follows (Chiao et al. 2011):

and for a trichromat:

$$
(\Delta S)^{2}=\frac{\left(\Delta Q_{1}-\Delta Q_{2}\right)^{2}}{e_{1}^{2}+e_{2}^{2}}
$$

$$
(\Delta S)^{2}=\frac{e_{1}^{2}\left(\Delta Q_{3}-\Delta Q_{2}\right)^{2}+e_{2}^{2}\left(\Delta Q_{3}-\Delta Q_{1}\right)^{2}+e_{3}^{2}\left(\Delta Q_{1}-\Delta Q_{2}\right)^{2}}{\left(e_{1} e_{2}\right)^{2}+\left(e_{1} e_{3}\right)^{2}+\left(e_{2} e_{3}\right)^{2}}
$$

where $\Delta Q_{i}=Q_{i, a}-Q_{i, b}$ is the difference between the quantum catch $Q_{i}$ of a stimuli $a$ and $b$ in the $i^{\text {th }}$ photoreceptor type. Quantum catch is found by

$$
Q_{i}=k \int_{\lambda \min }^{\lambda \max } I(\lambda) R(\lambda) S_{i}(\lambda) d \lambda
$$

where $\lambda_{\min }$ and $\lambda_{\max }$ are the bounds of the light spectrum of interest, $I(\lambda)$ is the spectrum of the incident light, $R(\lambda)$ is the reflectance spectrum of the surface being measured, $S_{i}(\lambda)$ is the spectral sensitivity of receptor type $i$, and $k$ is the von Kries adaptation constant, which is set to unity for this paper. The $e_{i}$ in Eq. 3.8\&3.9 represents noise in receptor type $i$ and can be approximated using the Weber fraction $\left(w_{i}\right)($ Chiao et al. 2011):

$$
w_{i}=\left(\frac{0.05}{\sqrt{n_{i}}}\right) \sqrt{n_{l w s}}
$$

where $n_{i}$ is the estimate of the proportion of the $i^{\text {th }}$ wavelength sensitive cone (i.e. short, medium, or long) and $n_{l w s}$ is the estimate of the proportion of the long wavelength sensitive cones (Chiao et al. 2011).

For the visual system parameters for the poison dart frog are taken from (Siddiqi et al. 2004) $\left(\lambda_{\max , S, M, L}=470,489,561 \mathrm{~nm} ; n_{l w s}=4, n_{m w s}=3, n_{s w s}=1\right)$ and those for the ferret are from (Isaac and Gregory 2013) $\left(\lambda_{\max , S, L}=430,558 \mathrm{~nm} ; n_{l w s}=\right.$ $\left.14, n_{s w s}=1\right)$. The spectral sensitivities corresponding to the $\lambda_{\max }$ values were calculated as described in (Akkaynak et al. 2013). 


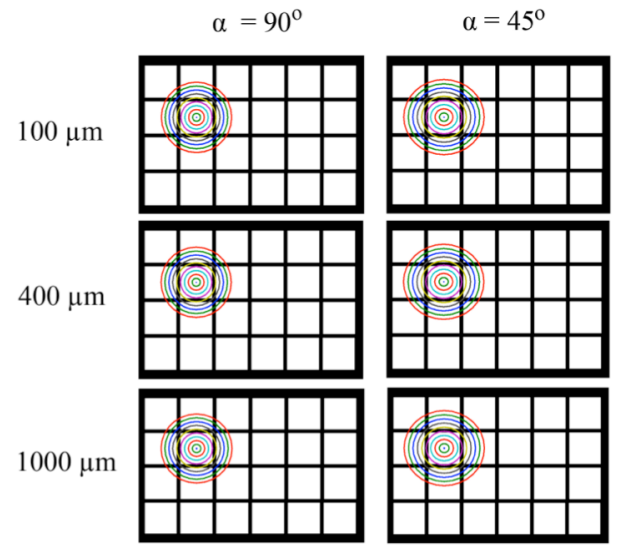

(a)
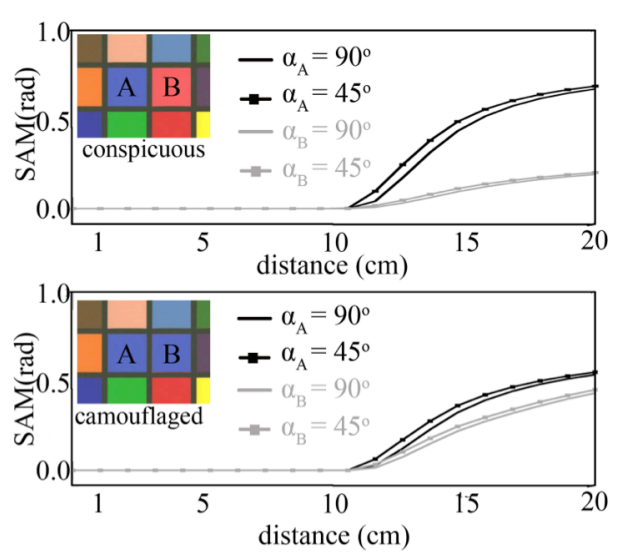

(b)

Figure 3.4 (a) When the optical fiber $(\mathrm{NA}=0.22)$ is held at $90^{\circ}$ to the surface being measured, the cross section of the cone of acceptance is a disk. As the measurement distance increases, the radius of the disk also increases. For a measurement at $45^{\circ}$, the cross section is an ellipse. The concentric curves show cross-sections at distances $0-20 \mathrm{~cm}$, in 10 equal steps. (b) Mathematical similarity of patches A (black lines) and B (gray lines) to their uncontaminated versions. Note that SAM only measures the similarity of the shape of the two spectra, disregarding magnitude. Results for different fiber diameters were similar and so only those for $100 \mu \mathrm{m}$ are shown here.

\subsection{Results}

In this section, the effect of $d$ and $\alpha$ on the spectral content of a signal is investigated by comparing the signal to its uncontaminated reflectance spectrum mathematically and then perceptually relative to two visual systems. For simplicity, it is assumed that the spectral measurements for the test stimuli are taken with the tip of the fiber pointing to the center of each patch, and that when measurements for two patches are being compared, they were recorded at the same $d$ and $\alpha$.

\subsubsection{Mathematical similarity of spectra}

In Figure 3.4a cross-sections of the cone of acceptance are shown for two measurement angles that are commonly used in animal coloration measurements, $90^{\circ}$ (cross-sections are circles) and $45^{\circ}$ (cross-sections are ellipses), respectively, with measurement distance varying from 0 to $20 \mathrm{~cm}$. The upper limit of $20 \mathrm{~cm}$ was chosen based on the author's experience - in general measurements taken from distances further than this are excluded from scientific analyses. In each case, it is assumed that the tip of the fiber is pointed at the center of patch A. Results vary little by fiber size, therefore they are only shown for the 100-micron fiber in the rest of the paper. In Figure 3.4b the similarity of the "contaminated" spectra of patches A and B relative to their "uncontaminated" versions (from Figure 3.3b) are shown using SAM (Eq. 3.7), for both measurement angles. The simulated patches are squares of $4.1 \mathrm{~cm}$, with a black boundary separating them, and in both the conspicuous and camouflaged case, the signal remains uncontaminated up to a measurement distance of about $10 \mathrm{~cm}$. Beyond that, the crosssectional sampling area expands into neighboring patches and there are two points to note. First, for a given measurement distance the degree of signal contamination depends on the measurement angle; the $45^{\circ}$ measurement has higher SAM values (i.e., more 
dissimilarity) then the $90^{\circ}$ measurement at any given distance. Second, the degree of contamination of spectra is not just a function of $d$ and $\alpha$, but also of the neighboring colors. Patches A and B have different neighboring patches. The reflectance spectrum of patch $B$ is more similar to the spectra of the patches that happen to be immediately adjacent to it than patch $A$ is to its neighbors. This is purely a result of the arrangement of color patches on the synthetic test target and affects the overall SAM scores for the selfsimilarity of patches A and B.
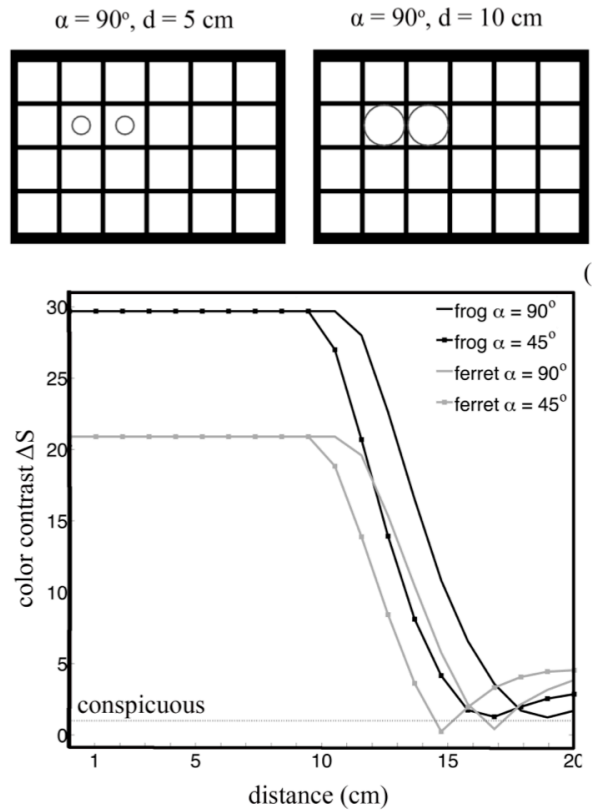

(b) $\alpha=90^{\circ}, \mathrm{d}=10 \mathrm{~cm}$

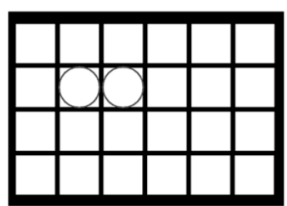

$\alpha=90^{\circ}, \mathrm{d}=15 \mathrm{~cm}$

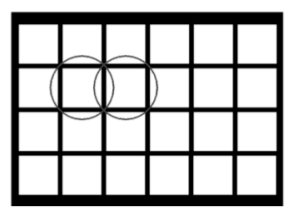

(a)

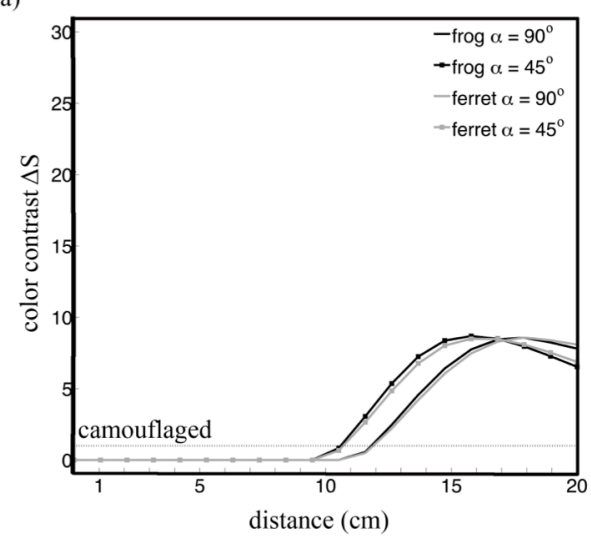

(c)

Figure 3.5 (a) Cross-section of the cone of acceptance when the fiber is held at an angle perpendicular to the surface, at measurement distances $5,10,15$ and $20 \mathrm{~cm}$ (the dimension of each square is $4.1 \mathrm{~cm}$ ). The signals measured from patches $A$ and $B$ are expected to remain pure up to $\mathrm{d}=10 \mathrm{~cm}$. (b) In the conspicuous case, patches $A$ and $B$ have little spectral overlap, and that translates to a high color contrast $(\Delta S)$ value for both the frog and the ferret. Beyond $d=10 \mathrm{~cm}$, the color contrast decreases (the signals become more similar) with both distance and measurement angle. (c) In the camouflaged case, the spectrum of patch $A$ is copied to the location of patch $B$. The color contrast is zero until $\mathbf{d}=10 \mathrm{~cm}$ and after that, the spectra quickly get contaminated. The signals measured at $\alpha=90^{\circ}$ remain slightly more pure than at $\alpha=45^{\circ}$. The dashed line indicates the just noticeable difference (JND) threshold of 1.

The same analysis is repeated with the spectra in patch B replaced with a copy of patch A to simulate an example where an animal might be trying to match the colors of its background to fool predators. Originally, patch A had little spectral similarity to patch B. Now, patch A has a neighbor with an identical spectrum, and its overall level of contamination falls compared to that in the conspicuous case. The new patch B (blue), however, has less spectral overlap with its neighbors than did the old patch B (red, case 1 ), and its overall level of contamination increases. This demonstrates that the measurement distance and angle alone are not enough to predict the level of contamination of a color signal recorded with a certain optical fiber, and that the neighboring colors, which are often difficult to predict in a biological application, also play a role. 


\subsubsection{Perceptual similarity of spectra}

\section{Case (1): Conspicuous animal}

Patches A and B were chosen to represent the pattern of a conspicuous animal because they have little spectral overlap. The receptor noise model would be expected to predict a high color contrast between these patches in the eyes of both the dichromatic ferret and the trichromatic frog. Figure 3.5a shows the cross sections of the cone of acceptance for the measurement of patches $\mathrm{A}$ and $\mathrm{B}$, at distances $d=5,10,15$ and $20 \mathrm{~cm}$. Beyond $d=10 \mathrm{~cm}$, the disks outgrow the square patches and we expect the spectral content of each patch to become contaminated with spectra from neighboring patches. This in turn affects the color contrast between the patches. This is indeed the case as seen in Figure 3.5b; the color contrast $(\Delta S)$ values for both animals are well above the just noticeable difference (JND) threshold of 1 (indicated by the dashed horizontal line) until $d=10 \mathrm{~cm}$, but the two spectra become increasingly similar (for both the frog and the ferret) after that point. If these measurements were taken at a distance of $15 \mathrm{~cm}$, the data could have led to an incorrect conclusion that the blue and red colors were indistinguishable $(\mathrm{JND}<1)$ to the ferret. Note that the $\Delta S$ for the frog never falls below the JND $=1$ line, meaning that these two patches, despite their spectral contamination, remain distinguishable to the trichromatic animal, which has the advantage of having an extra photoreceptor compared to the dichromate.

\section{Case (2): Camouflaged animal}

For the case of a perfectly camouflaged animal, patches A and B have identical reflectance spectra, but they have different neighboring colors (see Figure 3.4b inset). As expected, the spectra of patches remain identical when the measurements are uncontaminated, up to $d=10 \mathrm{~cm}$, and for both animals the color contrast is zero (Figure $3.5 \mathrm{c}$ ). Beyond that point, the contrast between spectra measured at $\alpha=90^{\circ}$ remains lower for a slightly higher $d$ value than it does for $\alpha=45^{\circ}$. Note that for this example, the magnitude of color contrast for both visual systems are comparable implying that the measurements of patch spectra (even though they were identical) are contaminated enough beyond $d=11 \mathrm{~cm}$ that the colors become distinguishable $(J \mathrm{ND}>1)$ to both the ferret and the frog. 


\subsection{Discussion}

Observations made in situ are key elements for understanding predator-prey interactions in the context of animal coloration and camouflage because our abilities to reproduce field conditions in the lab are limited. Thus, spectral data collected in situ from freely behaving animals in the wild and their habitat substrates contributes significantly towards our interpretation of camouflage patterns. However, these data are subject to quality issues not encountered in the lab. In laboratory setups, spectroscopic measurements are made through optical benches, which often contain vises and fiber holders fixing the positions of the sample and the measurement probes for consistent and accurate measurements. For field-based studies, standardizing data collection distance and angle is often impossible. Thus, spectral measurements of the same sample taken in the lab and in the field could be different because of variations in the measurement distance and angle, which depends on how close the surveyor can get to the animal whose colors are being measured. The equations presented in this chapter aim to provide a guideline for researchers so measurement errors made in the field could be quantified. The use of these equations are not limited to the field of animal coloration and camouflage since the ellipse representing the intersection of the acceptance cone and a surface only depends on fiber diameter and numerical aperture, which are provided by manufacturers, this method is generalizable to applications of spectroscopy in other fields.

\subsection{Conclusion}

Signal contamination varies based on the spectral content of the neighboring colors of a patch in addition to the measurement distance. Measurement distance was more important than measurement angle for the two angles considered $\left(45^{\circ}\right.$ and $\left.90^{\circ}\right)$. The measurement angle simulated (45 versus 90 degrees) does not appear to have a large effect on color measurement but $90^{\circ}$ may be preferable if the shape being measured has a $1: 1$ aspect ratio. Fiber size, at least in the range of 100-1000 micrometers, has a negligible effect on the field of view of the fiber. The patches used in this example were squares of $4.1 \mathrm{~cm}$, and for a feature of that size and shape, the maximum measurement distance that produced pure colors was approximately $10 \mathrm{~cm}$. Most features measured in the context of animal coloration are smaller than $4.1 \mathrm{~cm}$; for example the poison frog is only $1-2 \mathrm{~cm}$ in size (Siddiqi et al. 2004) and the largest body component of a European cuttlefish of mantle length $20 \mathrm{~cm}$ is around $3 \mathrm{~cm}$ (Akkaynak et al. 2013). Animals often have splotchy patterns with irregular shapes and non-Lambertian skins, and even though it may be possible to know the size of a feature that will be measured, there may be no a priori information regarding its neighboring colors. Equations 3.2-3.6 presented in this work could be used as guidelines before spectral measurements from animals or their habitats are made. 


\section{Chapter 4}

\section{Quantification of cuttlefish camouflage (S. officinalis): a study of color and luminance using in situ spectrometry ${ }^{3}$}

\subsection{Introduction}

Coleoid cephalopods are unrivaled in the animal kingdom in their ability to quickly and dynamically change their body patterns for signaling and camouflage (e.g., (Hanlon and Messenger 1988; Hanlon and Messenger 1996). Pattern and color change are achieved through the physiological control of chromatophore organs (Florey 1969; Messenger 2001a), and structural reflectors (iridophores and leucophores, (Mäthger et al. 2009; Wardill et al. 2012). Many laboratory and field studies have shown that camouflage behavior in cuttlefish is visually driven (e.g. (Chiao and Hanlon 2001b, a; Chiao et al. 2005; Barbosa et al. 2008a; Barbosa et al. 2008b; Barbosa et al. 2007; Chiao et al. 2007; Kelman et al. 2007; Allen et al. 2009; Chiao et al. 2009; Zylinski et al. 2009c, b, a; Chiao et al. 2010). Counterintuitively, these animals adaptively tune the colors of their body patterns in response to their visual backgrounds without the use of color vision (Marshall and Messenger 1996; Mäthger et al. 2006). It is natural to wonder how well cuttlefish can match the colors of substrates in their natural habitats if they cannot sense color with their eyes. Our observations in the laboratory and in the wild suggest cuttlefish color match is good enough to fool the human eye; but does it also fool the visual systems of predators?

In this work, we investigated how well the colors of cuttlefish skin matched the colors of substrates found in their natural habitats. Specifically, we quantified whether colors from cuttlefish camouflage patterns were discriminable from those of the background substrates in the eyes of predators. To do so, we developed a systematic analytical tool to compare the luminance and color contrast between animal and substrate spectra in the eyes of hypothetical di- and trichromatic fish predators and supplemented our spectral comparison by further analyzing cuttlefish color matching in human color space using the International Commission on Illumination (CIE) 1931 XYZ model (Wyszecki and Stiles 2000). In addition, we also used an auxiliary methodology because sometimes it may be necessary to assess the similarity of two spectra independently of the perception of a particular visual system. For example, there may not be enough information about the photoreceptor types or ratios of a certain predator, or a result that can be generalized across visual systems may be desired. In such cases, purely mathematical measures of spectral similarity can provide a rough approximation. For this reason, we used Spectral Angle Mapper (SAM), a technique commonly used to assess spectral shape similarity in the field of remote sensing (Yuhas et al. 1992), to quantify the similarity of cuttlefish and substrate spectra. We found that SAM correlated moderately with our biological measures of color contrast, making it comparable to a traditional chroma and hue based measure of spectral shape similarity (Endler 1990). This study is the first to collect in situ spectral data for color match assessment of European cuttlefish (S. officinalis).

${ }^{3}$ Parts of this chapter was previously published. See (Akkaynak et al. 2013) for full reference. 


\subsection{Background and related work}

Until now, spectrophotometric quantification of cuttlefish camouflage had only been performed using data collected in the laboratory (Mäthger et al. 2008;Chiao et al. 2011). Mäthger and colleagues (2008) were to first to quantitatively analyze the color matching abilities of cuttlefish. They measured reflectance spectra from 10 random points on animals $(\mathrm{N}=8)$ and background substrates in the laboratory and used normalized Euclidean distance to quantify spectral difference. They found that cuttlefish showed a better spectral match to the 11 natural substrates tested, than they did for the 2 artificial substrates, but did not analyze the discriminability of colors according to the visual systems of predators. Chiao and colleagues (2011) also performed a color match assessment in the laboratory, but they used a hyper-spectral imager to image body patterns. In addition, they modeled the visual systems of hypothetical predators and found that the colors cuttlefish produced were indistinguishable in the eyes of predators. Both studies found that cuttlefish colors showed a good mathematical and perceptual match to the substrates tested in the laboratory. However, substrates found in the natural habitats of cuttlefish show a more variation in terms of color and structure than those tested in the
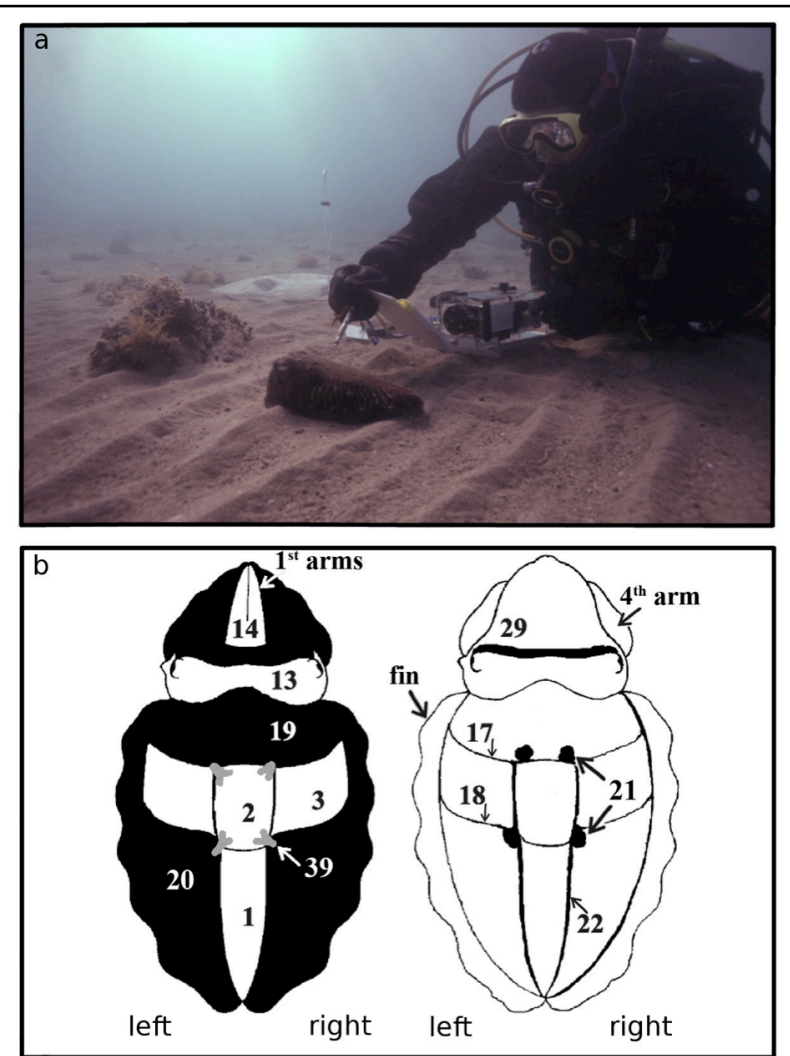

Figure 4.1 (a) Spectral data were collected by two divers (DA and JJA). One diver operated the spectrometer while the other took still photographs and video to document the sequence of measurements. All measurements were taken under natural daylight after the cuttlefish had habituated to the presence of divers. (b) Cuttlefish body pattern components measured in this study, numbered and capitalized according to their description in Hanlon and Messenger (1988). $1=$ white posterior triangle; $2=$ white square; $3=$ white mantle bar; $13=$ white head bar; $14=$ white arm triangle; $17=$ anterior transverse mantle line; $18=$ posterior transverse mantle line; 19 = anterior mantle bar; $20=$ posterior mantle bar; 21 = paired mantle spots; $22=$ median mantle stripe; $29=$ anterior head bar; $39=$ white square papillae.

laboratory. Therefore, in situ

measurements of cuttlefish skin radiance spectra under natural illumination and in natural habitats are crucial to provide empirical evidence to assess the degree of color matching.

In this study, we collected radiance spectra from the common European cuttlefish, Sepia officinalis, and their visual surrounds (sand, gravel, pebbles, algae-covered rocks, peacock's tail alga, hydrozoans, Pinna mollusc shells, etc., hereafter, "substrates") underwater on the Aegean Coast of Turkey. We describe the details of data collection, calibration and analysis next. 


\subsection{Methods}

Study site, animal \& substrate measurements

Seven cuttlefish (mantle lengths between 15 and $22 \mathrm{~cm}$ ) were studied off the village of Çeşmealtı (İzmir) on the Aegean coast of Turkey in spring, 2011. Fifteen radiance datasets were collected; each data set consisted of one animal, one location and one body pattern. Radiance measurements were taken using a USB2000 spectrometer (sensitivity range: 200-850nm; Ocean Optics, Dunedin, FL) coupled with a Compaq iPaQ handheld computer in a custom underwater housing (Wills Camera Housings, Victoria, Australia). Optical fibers (50 and 100-micron diameter) were used to collect data from cuttlefish and surrounding substrates (Figure 4.1a). We used a Spectralon (Labsphere, UK) target as a white reflectance standard. A CC3 cosine corrector (Ocean Optics, Dunedin, FL) was attached to the optical fiber for collection of irradiance data and an LS1-CAL tungsten light source (Ocean Optics) was used to obtain absolute intensity values. This calibration was done in a dark room to minimize stray light. Our equipment design is documented in (Roelfsema et al. 2006) and has been used for fieldwork by many others (Leiper et al. 2012; Hedley et al. 2012; Lim et al. 2009; Cheney et al. 2008; Matz et al. 2006; Cheney and Marshall 2009).

Photographs were taken with a 24-70 mm lens on a Canon EOS 1-Ds Mark II digital camera in a Subal housing equipped with a dome port. Videos recorded with a compact FlipCam (Irvine, CA) documented the underwater data collection. Measurements were taken under natural light, at depths shallower than 5 meters. Animals were carefully approached by two divers (DA \& JJA) until they habituated to the divers' presence and did not show any signaling behavior (e.g., unilaterally expressed pattern components or Paired Mantle Spots as part of a Deimatic display; see (Hanlon and Messenger 1988).

Animals were allowed to settle in a location of their choice and substrates were not altered, with one exception: before data acquisition, white rocks were placed near one cuttlefish to evoke a weak pattern (Figure $4.2 \mathrm{~g}$ ). This pattern is known to be the camouflage response to white cues in a cuttlefish's visual surrounds ( $S$. pharaonis, (Chiao and Hanlon 2001a); S.officinalis, e.g., (Barbosa et al. 2007; Mäthger et al. 2007).

In each case, ten to fifteen radiance measurements were recorded for each body component (Figure 4.2b), background element, or irradiance measurement and their average was used for the data analysis. If the cuttlefish moved or changed body patterns during data acquisition, the animal was allowed to re-habituate and measurements from the new location/body pattern were recorded as a new data set. Similarly, if lighting conditions changed significantly (e.g., in response to passing clouds), a new set of irradiance and white standard measurements was recorded. In both cases, we only analyzed datasets that were taken under consistent lighting conditions. In all cases, the probe head of optical fiber was less than $3 \mathrm{~cm}$ away from the feature whose spectrum was being recorded (see Chapter 3 ). We ensured that the diver's hand did not shadow the area of interest. 


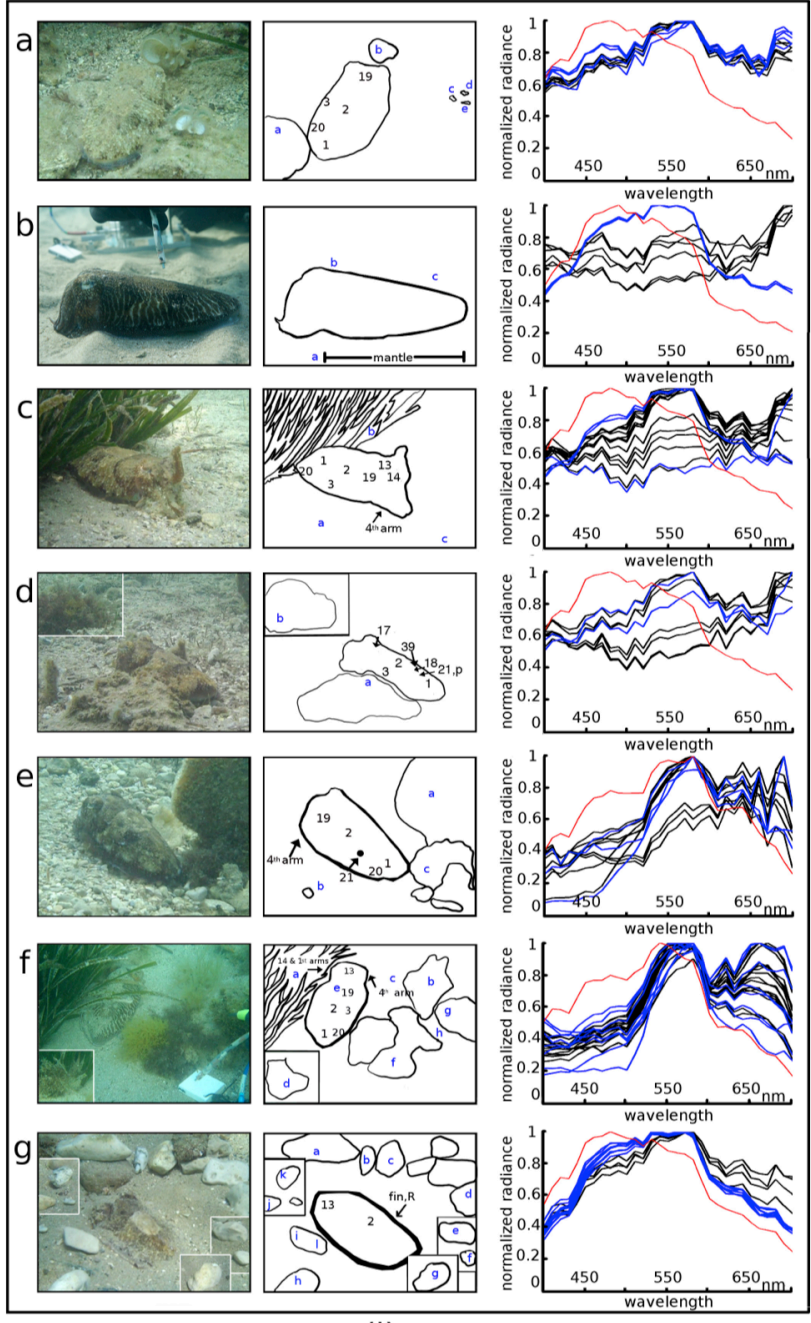

(i)

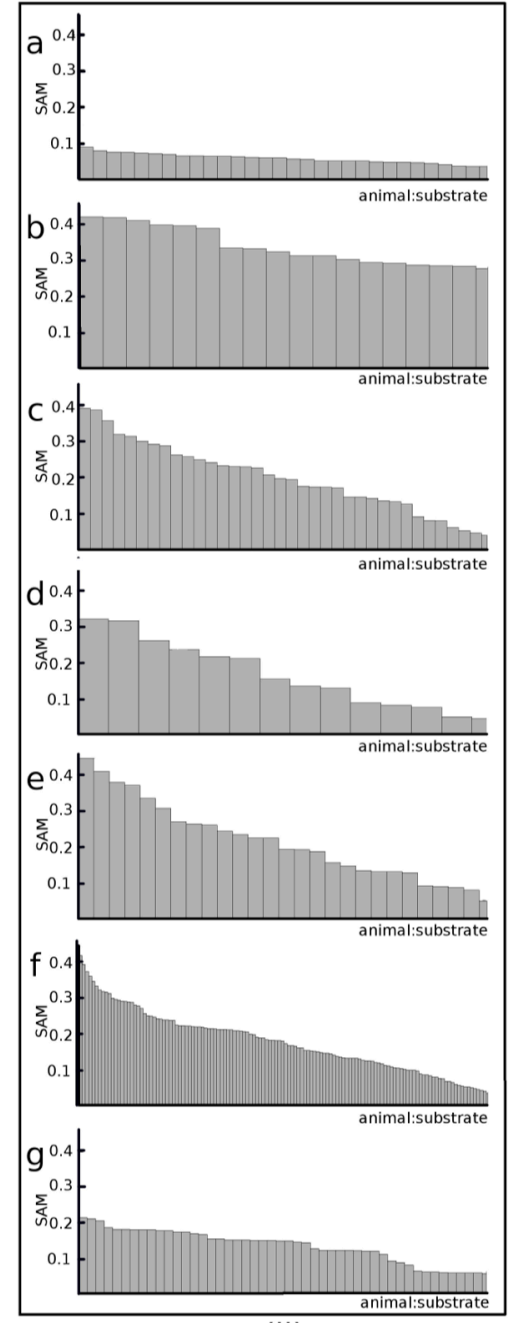

(ii)

Figure 4.2 (a-g) Presentation of seven datasets; in (i) we present a photograph of the scene, an outline of the scene showing the spots radiance spectra were recorded from and normalized radiance spectra we measured; in (ii) the Spectral Angle Mapper score computed between the spectra of each animal component and background substrate are shown. For example, for the animal in (a), the bars represent the SAM score between pairs of features: ("a") \& ("1"), ("a") \& ("2"), ("a") \& ("3"), ("a") \& ("19"), ("a") \& ("20"), ("b") \& ("1"), ("b") \& ("2"), ("b") \& ("3"), ("b") \& ("19"), ("b") \& ("20") and so on. They are sorted in descending order. See text for details. We repeat here the pattern names we have used in text for each photo: (a) uniform/stipple, (b) dark uniform with weak zebra stripes, (c) mottle/weak weak, (d) mottle, (e) mottle, (f) weak zebra, and (g) weak. See supplementary online resource 2 for larger, high resolution images. 
Assessment of chromatic similarity between animal and background spectra using a nonbiological measure (Spectral Angle Mapper)

In the field of remote sensing, automated spectral library search algorithms developed for hyper-spectral images (Chang 2003; Sweet 2003; Freek 2006; Nidamanuri and Zbell 2011) are used to compare reflectance spectra of known targets to those of novel spectra by computing a scalar similarity score between them. For these algorithms, stochastic methods are more frequently used than deterministic methods because imaging conditions can be imperfect and because the high spectral resolution of a hyper-spectral sensor often results in more than one material spectral signature in a given pixel. In our study, spectral data were point-by-point measurements of solid color patches where the area of the patch was much wider than the diameter of the spectrometer fiber. Hence, we use a deterministic method, Spectral Angle Mapper (SAM), from the field of hyperspectral image classification in our assessment of spectral shape similarity. SAM is the most commonly used spectral angle-based similarity measure ((Yuhas et al. 1992); (Kruse et al. 1993) and it is the normalized inner product of two vectors. It is computed as follows:

$$
\theta=\cos ^{-1}\left(\frac{S_{1}{ }^{T} S_{2}}{\left\|S_{1}\right\|\left\|S_{2}\right\|}\right)
$$

where " $\mathbf{S}_{1}$ " and " $\mathbf{S}_{2}$ " are the two spectra vectors being compared. Each continuous radiance spectrum is vectorized (denoted) into 31 dimensions by taking its value every 10 $\mathrm{nm}$ in the visible range (400-700nm). "T" denotes the transpose of a matrix and the $\|$. symbol denotes Euclidian norm; the division by the vector norms indicates that SAM is indifferent to the magnitude of the vectors (brightness) and only calculates similarity of spectral shape (color). A small angle between two vectors indicates that the spectra are similar in shape.

\section{Calculation of color difference using chroma and hue}

A common way to assess the similarity of two spectra independent of a visual system is to compute their chroma $(\mathrm{C})$, hue $(\mathrm{H})$ and brightness $(\mathrm{B})$ values, and calculate the Euclidean distance between them (D). A small Euclidean distance means that the two colors are similar. Here, we used the segment classification analysis of spectra from (Endler 1990) to calculate hue and chroma. Our goal is to compare the performance of this method to that of SAM. SAM metric does not take brightness into account and for a fair comparison, before chroma and hue values are calculated each spectra should be multiplied with a constant to equalize their overall brightness (see (Endler 1990) for further details). Following this, the distance between the two colors are found from:

$$
D=\sqrt{\left(H_{1}-H_{2}\right)^{2}+\left(C_{1}-C_{2}\right)^{2}+\left(B_{1}-B_{2}\right)^{2}}
$$

where $B_{1}=B_{2}$ due to the brightness equalization step carried out. 
Assessment of spectral similarity between animal and background in the eyes of hypothetical di-and trichromatic fish predators: luminance and color contrast

Although the details of visual systems of cuttlefish predators are not known (Serranus cabrilla is the only fish species observed directly to prey on S. officinalis in the Mediterranean sea), we may speculate that cuttlefish are preyed upon by a variety of vertebrate and invertebrate predators with different visual systems ((Hanlon and Messenger 1988; Hanlon and Messenger 1996). Thus, we chose one dichromatic fish and one trichromatic fish as their potential predators to simulate their views of these camouflaged cuttlefish. In dichromatic fish, the $\lambda_{\max }$ of $\mathrm{S}$ and $\mathrm{M}$ cones was 460 and 570 $\mathrm{nm}$. In trichromatic fish, the $\lambda_{\max }$ of S, M, and L cones was 460,540 , and $570 \mathrm{~nm}$. N.B., although the choice of these $\lambda_{\max }$ of dichromatic and trichromatic cones was arbitrary, shifting the $\lambda_{\max }$ of these cones up or down 10-20 nm did not visibly affect the results.

We carried out this analysis for photoreceptor ratios 1:1:1 and 1:2:2 for trichromats and 1:1 and 1:2 for dichromats; these are typical fish retina cone mosaic patterns (Shand et al. 1999; Cheney and Marshall 2009). The results of our analysis in both cases were similar, and we only present results for the ratios 1:1:1 for trichromats and 1:1 for dichromats.

Luminance contrast $(\Delta L)$ is calculated as $L_{1}-L_{2}$, where $L=\ln (k Q)$; $\mathrm{k}$ is the von-Kries adaptation coefficient and $Q$ is the quantum catch value. Spectra were collected under the same ambient conditions for each dataset, therefore the same vonKries coefficient was applied to both luminance values $\left(k_{1}=k_{2}\right)$. Thus, $\Delta L=\ln \frac{k_{1} Q_{1}}{k_{2} Q_{2}}=\ln \frac{Q_{1}}{Q_{2}}$. Quantum catch value is computed as follows:

$$
Q_{i}=\int_{\lambda \min }^{\lambda \max } R(\lambda) I(\lambda) S_{i}(\lambda) d \lambda
$$

where $R(\lambda)$ is the reflectance spectrum of the feature of interest, $I(\lambda)$ is the spectrum of the light source, $S_{i}(\lambda)$ is the spectral sensitivity of the $i^{\text {th }}$ photoreceptor (i=S,M,L), $\lambda_{\min }$ and $\lambda_{\max }$ are the bounds of the visible spectrum, respectively. $S_{i}(\lambda)$ was based on the visual pigment template of Stavenga et al. (1993) with beta-peak shifted linearly, after Palacios et al. (1996) using the equation generated by Hart (1998). For calculating luminance contrast, we only used the long wavelength receptors since they are thought to be responsible for luminance contrast in fish (Marshall et al. 2003a). We computed luminance contrast between the cuttlefish body parts, as well as between cuttlefish parts and background substrates. For color contrast, we used the metric $\Delta S$ (with Weber fraction 0.05) as defined by the Vorobyev-Osorio Receptor Noise Model (Vorobyev and Osorio 1998). 


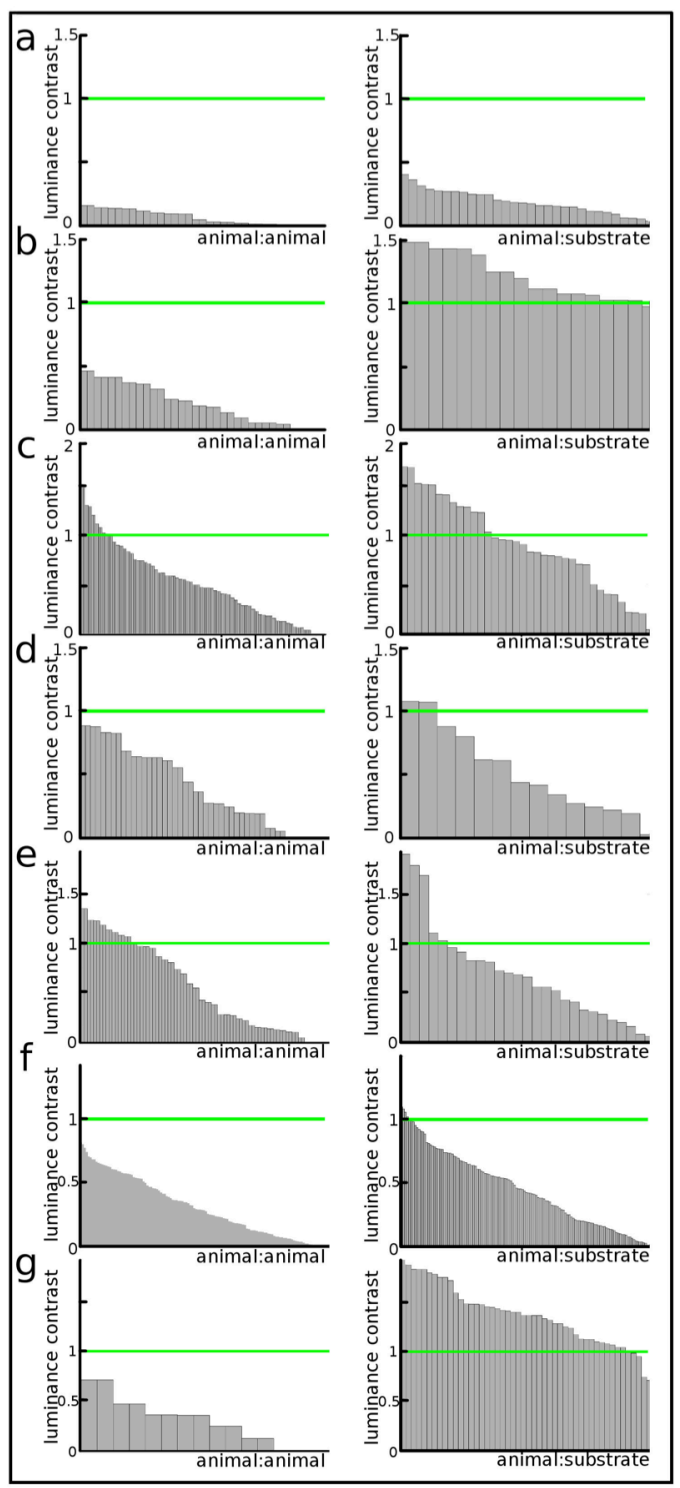

(i)

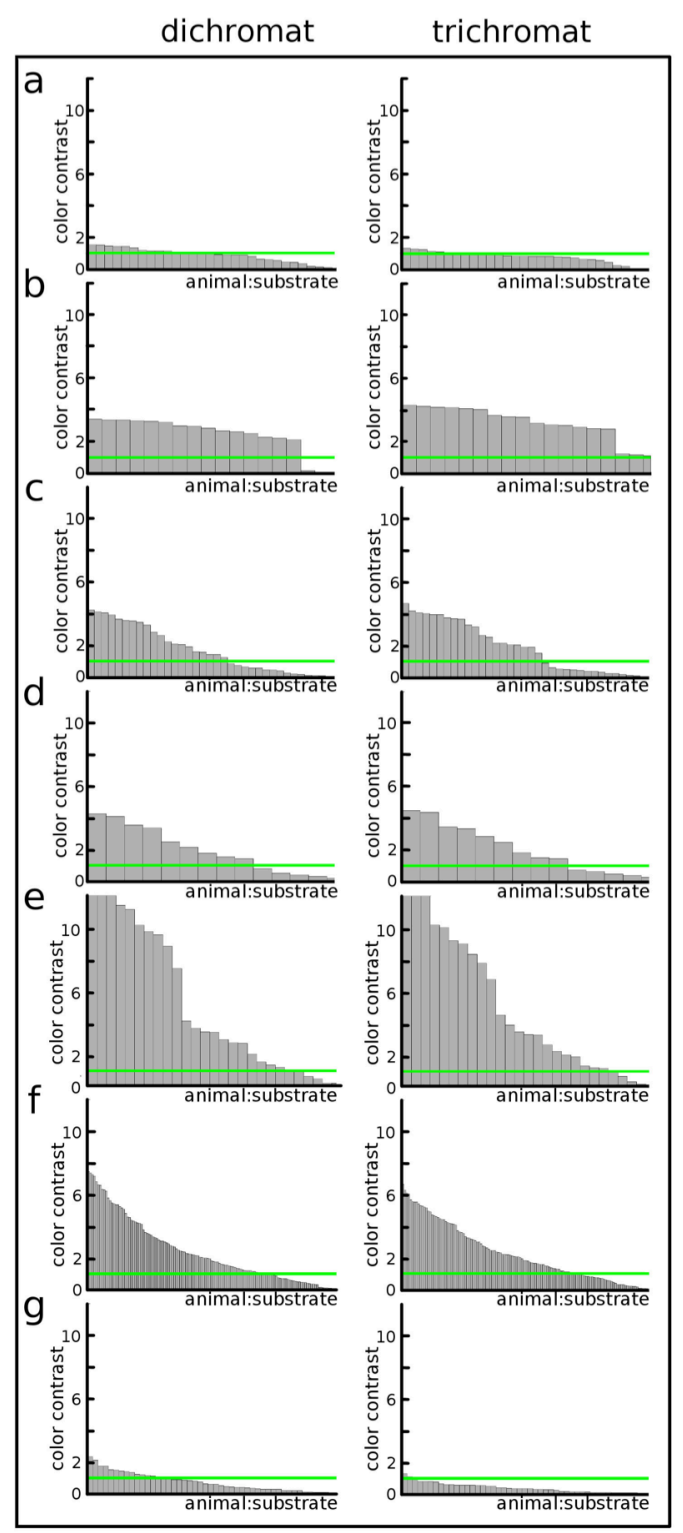

(ii)

Figure 4.3 (a-g) In (i), luminance contrast is shown for two cases: (1) amongst the spectra of the cuttlefish body components; (2) between the spectra cuttlefish components and the background substrates. In (ii), color contrast calculated between spectra of cuttlefish components and substrates are presented for hypothetical di and tri-chromats we modelled. Luminance contrast values shown are for trichromats only and are identical for dichromats. See text for details. In all plots, the green line indicates a "just noticable difference" of 1 ; pairs of features that fall below this value cannot be distinguished by the visual system under consideration. 
Visualization of spectral similarity between cuttlefish and backgrounds in human and hypothetical fish predator color spaces

It is helpful to visualize the spectral match between a cuttlefish and its surroundings as a human would have seen it. We used the CIE 1931 (2 degree observer) color matching functions to obtain XYZ tri-stimulus values from radiance spectra. We then plotted the xy-coordinates of the tri-stimulus values of cuttlefish body components and substrates on a CIE 1931 Chromaticity Diagram; see (Wyszecki and Stiles 2000) for details. For comparison, we included the xy-coordinates corresponding to the radiance spectra of the 24 patches of a Macbeth ColorChecker (Xrite, Inc. Grand Rapids, MI), assumed to be viewed under a CIE D65 illuminant (noon daylight, 6504K). Patches of a Macbeth ColorChecker are designed to represent colors humans encounter on a daily basis. N.B.: the CIE XYZ chromaticity diagram is not perceptually uniform; therefore, the same distance between two pairs of colors on the XYZ diagram may correspond to different perceptual distances. The XYZ tri-stimulus values cannot be visualized directly; to offer a visual comparison of the variety of cuttlefish and habitat colors at our field site, we converted the XYZ tri-stimulus values into the sRGB space and created color patch assemblies for both animal and substrate data. For details on converting XYZ tri-stimulus values to RGB color spaces, see (Reinhard et al. 2008)).

Chromaticity diagrams are also used in the studies of animal color vision (see (Pike 2011a) and (Kelber et al. 2003) as graphical representations of perceived colors. We followed the methodology described in (Kelber et al. 2003) to plot the loci of colors measured from cuttlefish and surrounding substrate on the Maxwell triangle for a hypothetical trichromatic observer with $\lambda_{\max }$ values for $\mathrm{S}, \mathrm{M}$, and L cones 460,540 , and $570 \mathrm{~nm}$.

\section{Simulation of color and luminance contrasts at depth}

Underwater, the available light field changes in intensity and spectral composition, changing the appearance of objects in response to factors such as: depth, time of day, weather conditions and the amount of suspended particles in the water column. To illustrate the effect of this change on camouflage, data from a uniform dark animal collected at $1 \mathrm{~m}$ depth (an animal that did not appear well color matched to its surroundings) were used to simulate the appearance of its colors at a depth of $10 \mathrm{~m}$ using irradiance spectra collected at $10 \mathrm{~m}$ at our study site. The simulation was done as follows. First, the radiance spectra from the original dataset were converted into reflectance by dividing the difference of radiance of the feature of interest and dark noise by the difference of white standard radiance and dark noise. Second, these reflectance spectra were multiplied with irradiance spectra we recorded in situ at 10 meters depth. Third, simulated animal and substrate radiance spectra were assessed for similarity and discriminability using methodology described above. Color patches were simulated in sRGB space using the tri-stimulus values obtained from the CIE 1931 XYZ model.

\subsection{Results}

In Figure 4.3i radiance measurements taken in the field are summarized. Measurements taken from cuttlefish components are labeled on the outlines of photographs with black numbers, those taken from substrates are labeled with blue letters. The same color convention is used in spectral curves and each curve is normalized 
by its maximum value to emphasize similarity of shape. The red curve in each plot is the (normalized) spectrum recorded from a Spectralon target and represents the shape of "white" under ambient light conditions.

Use of Spectral Angle Mapper for assessing spectral shape similarity between animal and background

Figure 4.3ii shows the Spectral Angle Mapper scores computed between each cuttlefish component and background substrate measured. The limits of each plot were adjusted to the minimum and maximum values of SAM encountered across all datasets $(0$ and 0.4508 , respectively). The score computed by SAM is a measure of how well two multi-dimensional vectors are aligned and does not carry a biological meaning. Therefore, while the SAM scores cannot give any information regarding the discriminability of two colors from the perspective of any visual system, they are informative about how similar the shapes of two spectra are, which can be compared to chromaticity. In general, the lower the SAM score, the more similar the colors. For example, in Figure 4.2a, the cuttlefish has a uniform body pattern, which appears well matched to the surrounding substrates in color. The corresponding SAM plot has low scores throughout. The animal in Figure $4.2 \mathrm{~b}$ also has a uniform pattern but, unlike Figure 4.2a, it does not match the surrounding sand. This difference is captured in the magnitude of the values displayed in the SAM plots.

\section{Assessment of luminance and color contrasts in the eyes of hypothetical di-and trichromatic fish predators}

In Figure 4.3i, we present luminance contrast among cuttlefish components and luminance contrast between cuttlefish components and substrates. Contrast among cuttlefish components is an indicator of whether the cuttlefish pattern is uniform or nonuniform (i.e. mottle, zebra or weak), and contrast between animal and substrate is an indicator of how similar the cuttlefish components are from the substrate in brightness. Figure 4.3ii shows color contrast between cuttlefish and substrates as seen by hypothetical di- and trichromats. In both (i) \& (ii), a value of 1 is a "just noticeable difference" (JND) and is marked with a green line.

Pattern 1 (Figure 4.3a). Luminance contrast values below the JND $=1$ line suggest this animal has a uniform pattern, and matches the substrates in intensity. The photograph in Fig. 2a confirms these observations. Low color contrast values suggest that this animal is difficult for both di- and trichromats to distinguish from its background.

Pattern 2 (Figure 4.3b). Low luminance contrast among animal components indicates a uniform pattern. Almost all cases of luminance contrast between animal and substrates are above the visual threshold, indicating this animal did not match the substrate in luminance. Color contrast values suggest this animal can be distinguished from its background by both di- and trichromats, but note that color contrast values, overall, are lower for the dichromat (on average, $\Delta S_{t r i}-\Delta S_{d i} \approx 0.7$ ) indicating that it is harder for the dichromat to detect this animal when compared to a trichromat. Indeed, there was a mismatch between this cuttlefish in a dark uniform body pattern (with weak zebra stripes) and the sand in its immediate surroundings as confirmed by Figure $4.2 \mathrm{~b}$. 
Pattern 3 (Figure 4.3c). In this dataset, we observed that cuttlefish components had high and low luminance contrast values among themselves indicative of a non-uniform (mottle, zebra or weak pattern). The photograph in Figure 4.3c shows a mottle/weak weak body pattern. In both color and luminance contrast, when compared to the dark green Posedonia seagrass ("b"), all cuttlefish components scored above the visual threshold. In response to visual cues from a nearby three-dimensional structure with projections approximately the width of its arms, this animal raised its first pair of arms, a postural component of cuttlefish camouflage (Barbosa et al. 2012). Although some body components matched the gravel in luminance and color contrast, the arm posture and overall body pattern suggested this animal may have performed masquerade camouflage in response to visual cues from the three dimensional Posedonia seagrass (for discussions of masquerade camouflage, see (Stevens and Merilaita 2009; Skelhorn et al. 2010; Buresch et al. 2011; Skelhorn and Ruxton 2011).

Pattern 4 (Figure 4.3d). Luminance contrast plots suggest this animal has a uniform pattern and matches the substrate in intensity. The photograph in Figure $4.3 \mathrm{~d}$ shows that this animal had a uniform body pattern with some aspects of a mottle. While this animal is well matched to its surroundings in luminance, its overall pattern is detectable in terms of color contrast to both di- and trichromats (Figure 4.3d,ii). It was difficult to distinguish this cuttlefish from nearby 3D objects, suggesting this animal might have performed masquerade camouflage instead of background matching (e.g., (Buresch et al. 2011). Note that the radiance spectra measured from the components of this animal were similar to those measured from a dark brown hydrozoan roughly two mantle lengths away (Figure 4.3d, inset).

Pattern 5 (Figure 4.3e). The luminance contrast values suggest a mottle, zebra, or weak pattern and the mostly high color contrast values in (ii) suggest this animal may be easy to spot against the background. The photo shows that this animal had a mottle pattern with weak expression of the White square and Median mantle stripes, components often seen in weak body patterns. All animal body components closely matched the nearby Pinna mollusc shell ("a") in luminance contrast but had high contrast when compared to the gravel ("b"). Overall, this animal could be detected by a hypothetical di-or trichromatic predator.

Pattern 6 (Figure 4.3f). The low values of luminance contrast among animal components suggest a uniform pattern. The photograph shows that the animal actually had a weak zebra pattern, showing low overall contrast. This animal allowed us to collect data from individual light and dark stripes within two body pattern components: the Posterior mantle bar ("20") and the White mantle bar ("3"). As in the case of pattern 3, the cuttlefish components better matched the green Posedonia seagrass in luminance than in color contrast (substrates " $a$ " and " $b$ "). This result is intuitive as the cuttlefish is not capable of producing a green color with its pigmented chromatophores, but can alter the intensity of skin to appear light or dark. Overall, this animal could be detected by a hypothetical di-or tri-chromatic predator. 
Pattern 7 (Figure 4.3g). Luminance contrast among cuttlefish components suggest that this animal has a uniform body pattern; however from Figure 4.2g, we see that it had a weak pattern. Its weak pattern was turned on after white rocks were placed nearby. When compared among cuttlefish components and substrates, luminance contrast values were high; indicating the animal's components are darker than the surrounding white rocks, with the exception of its White square. The luminance of the White Square ("2") was similar to the luminance of the other components. The cuttlefish components were most similar to substrates ("c" and "d") in luminance contrast, and nearly indistinguishable in the eyes of both diand trichromats in terms of color contrast. Note that the White square was not pure white, rather, closer to a light brown color. White square luminance and color are modulated by pigmented brown, orange and yellow chromatophore organs overlying the structural reflectors (lecuophores) responsible for whiteness in this animal (Mäthger et al. 2009).

\section{Comparison across all patterns}

Figure 4.4a summarizes the mean JND values across all datasets. In almost all cases, the dichromat JND values were equal to or smaller than the trichromat JND values, suggesting that it was more difficult for the dichromats to distinguish the cuttlefish components from the substrates. Similarly, the mean
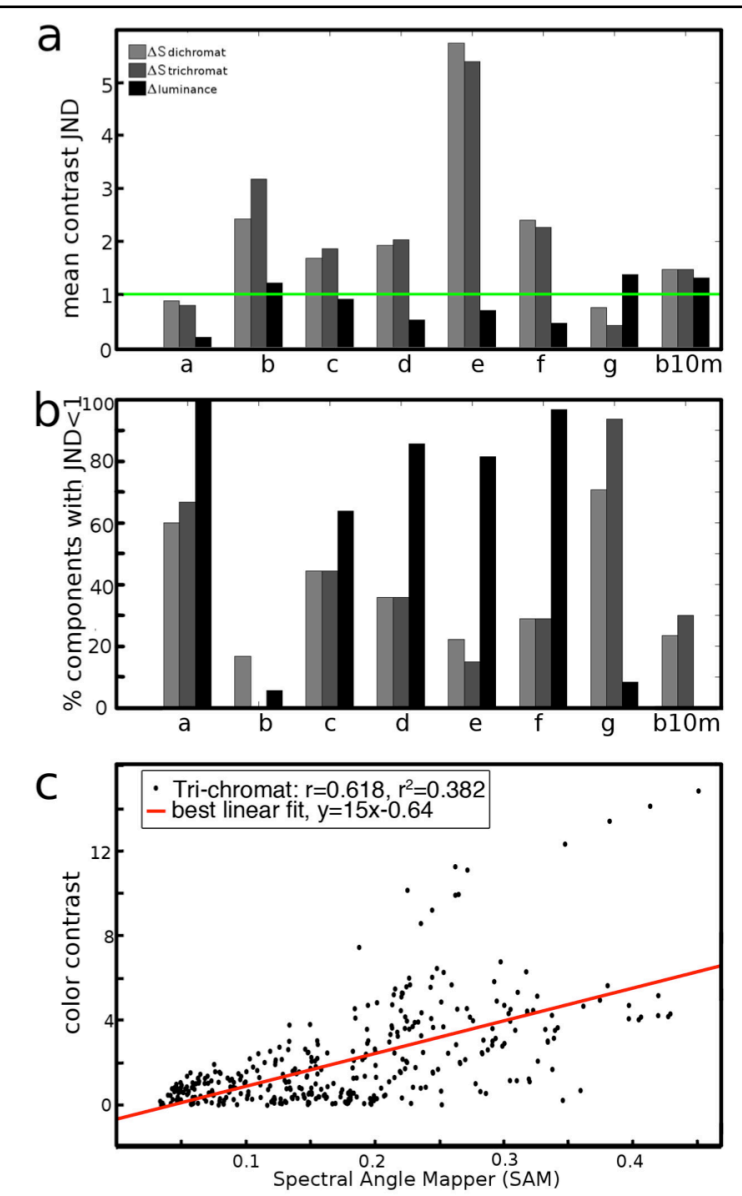

Figure 4.4 (a) Mean just noticable difference (JND) values shown for each dataset and (b) the percent of cuttlefish components that are below 1 JND for each dataset. (b) We found that SAM has moderate correlation $(0.5<|\mathbf{r}|<0.7)$ to $\Delta S$, a biological measure of color contrast for the data we present. Note that variance in the predicted color conrtrast score increases with increasing spectral angle scores (heteroscedasticity). This level of correlation did not change when we varied the photoreceptor ratios, and shifted peak wavelengths of $S, M$ and $L$ cone types by $10-20 \mathrm{~nm}$ for the visual systems we tested. This is comparable to $D$, a chroma and hue based spectral similarity metric which is independent of a particular observer's visual system. For the data we present, $D$ values were also moderately correlated with $\Delta S$. Data shown above for $\lambda_{S}=450, \lambda_{M}=530$ and $\lambda_{L}=560 \mathrm{~nm} ; r_{S A M, \Delta S}=0.618$ and $r_{D, \Delta S}=0.608$ (not plotted).

luminance contrast of the cuttlefish components, when compared to the surrounding substrate, was less than or slightly higher than $1 \mathrm{JND}$; the cuttlefish generally did a good job matching the luminance of the substrates. For datasets "b" and "b10m" (the latter is the animal whose appearance is simulated at 10 meters, described in the "Simulation of spectra at depth" subsection), the luminance contrast was nearly unchanged, while the mean color contrast fell to the JND $=1$ limit. The mean values in dataset "e" are likely to 
be affected by the measurement of the dark eyespots; these spots do not usually appear as a part of a camouflage pattern and were displayed as a warning to the divers.

Figure $4.4 \mathrm{~b}$ shows the percentage of pairs of components that had JND values less than 1. Animals in datasets "a" and "c" appear to have the best overall color and luminance match in the eyes of both di- and trichromatic predators, and those in "d", "e" and "f" seem to have done a better job matching luminance than color. Overall, the animal in dataset " $b$ " was the worst color matched animal. Its simulated appearance at 10 meters depth was more conspicuous than the animal at 1-meter depth.

The relationship between $S A M$, chroma and hue based color difference (D), and color contrast $(\Delta S)$

The SAM scores computed between cuttlefish components and substrates for all datasets showed moderate correlation to the color contrast values computed using the $(\Delta S)$ metric; the absolute value of the correlation coefficient varied between 0.5 and 0.7 $(p \approx 0)$ for all cases of $\lambda_{\max }$ and photoreceptor ratios we tested (Figure $4.4 \mathrm{c}$ ). Note that the data appear to be heteroscedastic. $\mathrm{D}$, the color difference computed using chroma and hue values, also correlated moderately with $\Delta S$. When compared against each other however, SAM and D had a weak a correlation (on average, $\rho \approx 0.4$ ). When assessed individually, on average SAM was moderately correlated with hue, and weakly correlated with chroma.

Visualization of spectral similarity between cuttlefish and backgrounds in human color space

We plotted the loci of $x y$ values for each animal component and substrate on a Chromaticity Diagram (Figure 4.5a). Cuttlefish body components and substrates were constrained to a general area around the point $x \approx 0.4 \& y \approx 0.35$ near the locus of gray colors at $\mathrm{x}=0.33, \mathrm{y}=0.33$ (the "white point" or the "achromatic point"). All spectra measured from cuttlefish body pattern components, and spectra, were converted to sRGB colors and are shown with a corresponding plot of "white" (a highly reflective Spectralon white standard) under ambient light conditions (Figure 4.5b). This visualization revealed that the colors of both the animals and substrates at our study site were limited to a narrow range within the gamut of colors visible to humans.

\section{Simulation of spectra at depth}

One cuttlefish (see Figure 4.2b; data taken at $1 \mathrm{~m}$ depth) poorly matched its surrounding sandy substrate. We used the irradiance profile we recorded at our study site at a depth of 10 meters to obtain a theoretical ambient light field and simulate this animal's radiance spectra had they been collected at that location (see Methods for details). Simulated spectra, SAM, luminance and color contrast results are presented in Figure 4.4a. At this depth, the shapes of all spectra become similar in shape due to the ambient light conditions as shown by the spectral curves. Overall, the SAM scores decreased by about $50 \%$; indicating that the spectra became more similar in shape at depth. Luminance contrast remained nearly the same. This is expected, as luminance contrast is a ratio of the quantum catches, and remains unchanged when the same process attenuates both spectra. The color contrast plots show that in the eyes of both di- and 
trichromat, the color match at $10 \mathrm{~m}$ depth has gotten better, and it is now harder to distinguish this animal from its background.

In Figure 4.6b Maxwell triangles show the change in the appearance of colors from $1 \mathrm{~m}$ to $10 \mathrm{~m}$. At 10 meters, cuttlefish and substrate colors form tighter clusters than they did at 1-meter depth. The sRGB appearance of colors shows that at 10 meters, the cuttlefish and substrate colors are indistinguishable.

\subsection{Discussion}

The utility of in situ spectrometry in studying animal camouflage

Here we presented spectrophotometric field data collected from camouflaged cuttlefish (Sepia officinalis) and some of their surrounding substrates. We have advanced previous studies by collecting data in the cuttlefish's natural benthic, near-shore environment where the daylight spectrum is affected by atmospheric conditions, water quality and depth (Jerlov 1968, Jerlov, 1976 \#205; Tyler and Smith 1970). Studying cuttlefish camouflage under natural conditions is essential for the study of animal and
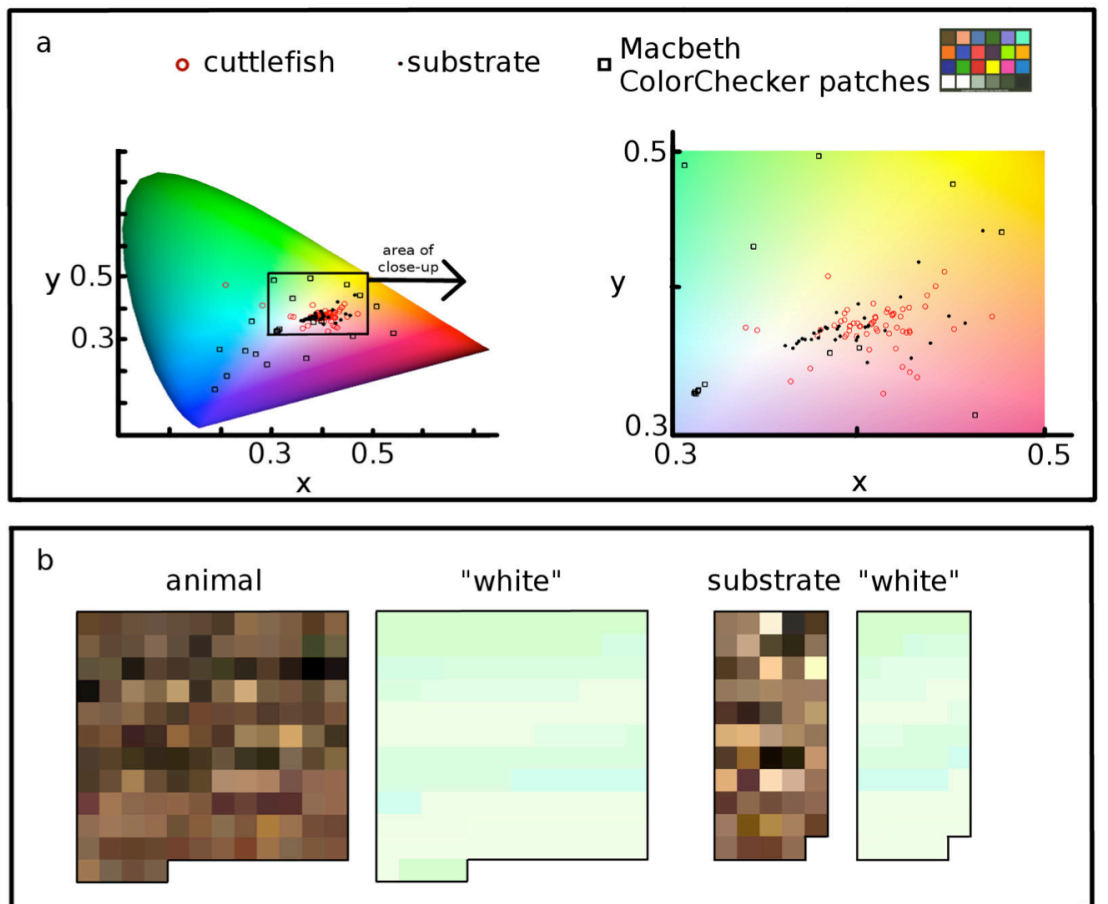

Figure 4.5 Analysis of cuttlefish color matching with respect to the human visual system. (a) Chromaticity plot showing the loci of cuttlefish and substrate $x y$ chromaticity coordinates obtained from radiance spectra measured in the field. Black squares show the xy coordinates of the patches of a Macbeth ColorChecker. (b) Color assemblies of all cuttlefish components measured. Each color patch in an assembly is accompanied by a corresponding white patch, representing the way "white" looked under the ambient conditions. Depending on depth, time of day, visibility etc., white may appear as shades of green and blue.

background luminance and color because it is under these conditions that cuttlefish camouflage body patterns have evolved to successfully deceive the eyes of predators. 
Recently, field spectrometry data collected by Hanlon et al. (2013) were analyzed for a different species, the giant Australian cuttlefish (Sepia apama), using a similar approach to quantify camouflage in the eyes of potential fish predators. Studies assessing the color signals of animals through the visual systems of their known predators have been done for chameleons (Stuart-Fox and Moussalli 2008), spiders (Heiling et al. 2003), (Théry and Casas 2002), fish (Marshall and Vorobyev 2003) and birds (Endler and Mielke Jr 2005). Spectrometers, however, are not ideal instruments for the assessment of overall animal patterns because they only record point-by-point samples. This makes it difficult, if not impossible, to collect spatial information from high-frequency textures, i.e. textures that are not solid color patches. In addition, animal coloration studies using spectrometers require getting the optical fiber very close to the skin of the animal; this is challenging while studying freely behaving animals in their natural habitats. The use of such equipment underwater further complicates the data collection process due to the rapidly changing light field from the undulations of the sea surface, limited light penetration at depth, particles suspended in water that affect visibility. Furthermore, wave surge, currents, and practicality issues such as bulky watertight housings affect the speed and maneuverability of divers, and the corrosion of equipment from seawater and reduced performance from most batteries due to low operating temperatures also distinguish such marine studies from terrestrial endeavors.

Imagers that record continuous spectra for every pixel in an image, namely multiand hyper-spectral cameras, are becoming common in many fields of science, and their use has been pioneered by (Chiao et al. 2011) in the field of animal coloration. While the costs of such imagers are still prohibitive, rapid developments in technology are making their deployment in the field as fast as commercial off-the-shelf digital cameras, and they will likely replace spectrometers to become the standard in studies of coloration in the next decade.

\section{A non-biological measure, $S A M$, is a rough estimate of color contrast, $\Delta S$}

Little is known about the predators of $S$. officinalis and their visual systems.

While retinal properties of most fish eyes are known, this information does not necessarily represent how they perceive scenes; and it most cases, it is difficult to do psychophysics experiments with fish to develop better models of their visual systems. Here, we show that a purely mathematical spectral shape similarity measure, SAM, is moderately correlated to the biological models of color contrast we used. This level of correlation holds for di- and trichromats, as well as the range of photoreceptor ratios and S, M, L peak wavelengths we tested for. In cases where there may not be enough information about the visual system of a particular observer, or a result that can be generalized across observers is desired, SAM could be used to score the similarity of shape between two spectra (shape similarity roughly corresponds to a similarity in chromaticity). Traditionally an observer-independent color difference metric D (Eq. 4.2) based on hue and chroma is used to estimate similarity of spectral shape (Endler 1990). We found that this measure also has a moderate degree of correlation to the color contrast values computed here. SAM could be computed alongside hue and chroma whenever a rough and objective spectral similarity score needs to be calculated. For our datasets, SAM and hue were better correlated than SAM and chroma; SAM and D were weakly correlated. SAM and D individually correlate moderately with color contrast while they 
are weakly correlated with each other; this may be because of their different working mechanisms. The segment classification method takes advantage of the opponency of color channels while SAM is only mathematical measure of shape. These results should be interpreted with caution since they do not relate to any biological system and most terms such as luminance, color, chromaticity etc. are meaningful with respect to visual systems. More specifically, they don't refer to the visibility of colors. We expect a more solid understanding of the relationship between mathematical and biological measures of color contrast to emerge as future studies adopt the calculation of a SAM score along with their biological analysis.
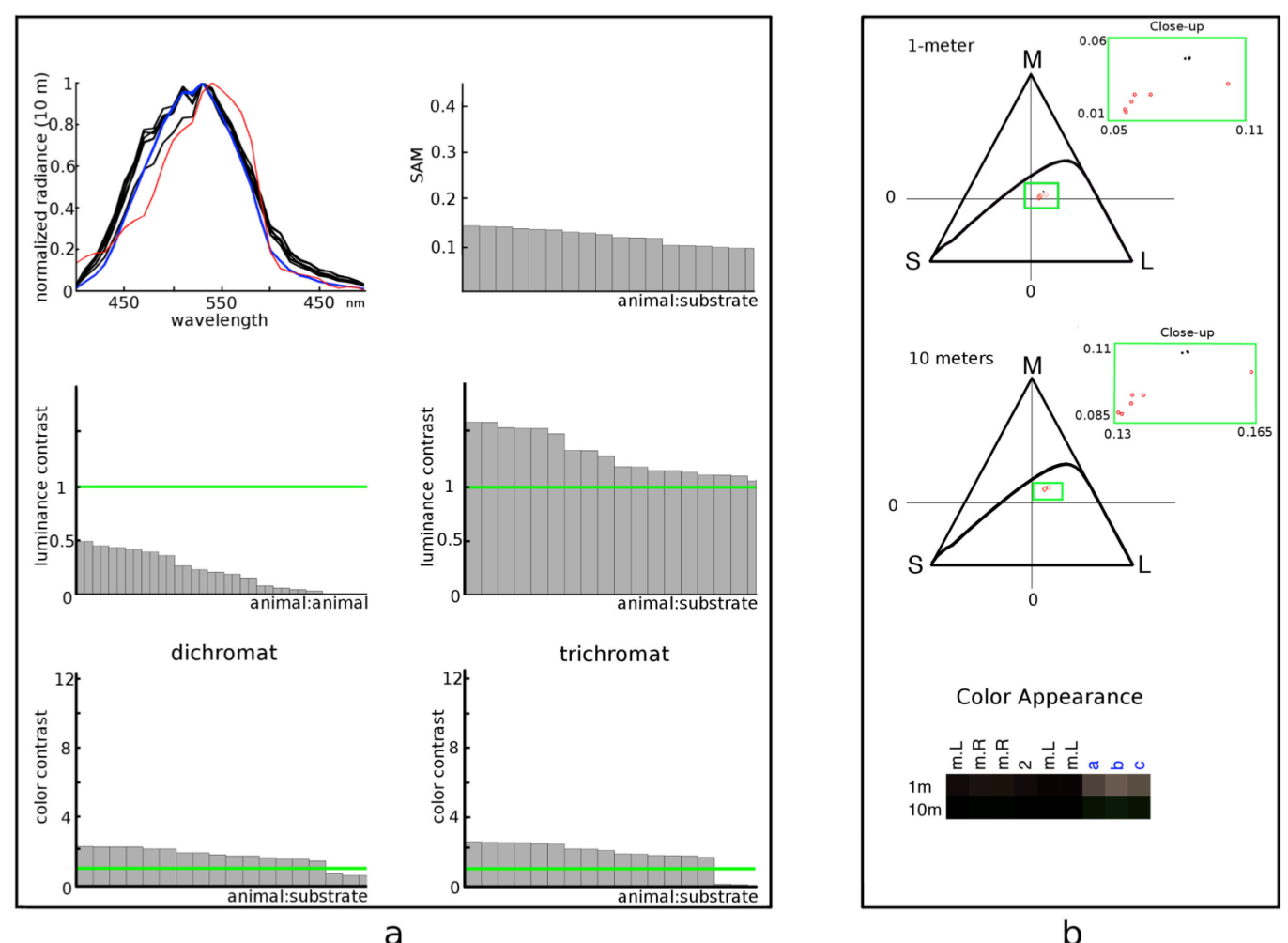

Figure 4.6 Simulation of the spectra of the animal and nearby substrate from Fig. $2 \& 3 b$, at a depth of 10m: (a) Normalized radiance spectra of cuttlefish components and substrates; red line indicates normalized spectra from of a Spectralon white target; SAM scores are almost halved when compared to Fig. 3b; luminance contrast remains unchanged and color contrast has decreased significantly. (b) Visualization of the colors of cuttlefish and substrate patches on a Maxwell triangle. In the $1 \mathrm{~m}$ case the loci of colors are relatively widespread, but they become almost coincident at $10 \mathrm{~m}$. sRGB representation of color appearance suggests colors are indistinguishable to the human visual system at 10 meters depth.

\section{Assessment of spectral similarity between cuttlefish and background in human and theoretical fish predator color space}

The spectral similarity between cuttlefish and their surroundings was analyzed from the perspective of the human visual system. We calculated the tri-stimulus values of each spectrum using CIE 1931 XYZ color space and plotted these tri-stimulus values of cuttlefish body pattern components and surrounding substrates on an iso-luminant 
chromaticity diagram tuned for the human visual system. This visual representation provided a way to assess the similarity of spectral properties between animal and background. Cuttlefish body components and substrates, were constrained to a general area around the point $x \approx 0.4 \& y \approx 0.35$ near the locus of the "white point" at $x=0.33, y=$ 0.33 (Figure 4.5a). Colors close to this point would appear gray under most lighting conditions. This narrow distribution implies that while cuttlefish chromaticity values are remarkably close to those of substrates, our study site was not very colorful, at least in comparison with terrestrial colors that humans are used to (see black squares that show colors from a Macbeth ColorChecker, representative of colors that a human might observe terrestrially on a daily basis).

In our analysis of luminance contrast, we only presented results for trichromats. Since long- wavelength receptors are thought to be responsible for luminance contrast in fish (Marshall et al. 2003b) and we used $\lambda_{\max }=570 \mathrm{~nm}$ for both the $\mathrm{L}$ cone for the hypothetical trichromatic predator and the $\mathrm{M}$ cone for the dichromat, no differences were expected. Point-by-point spectrometer data do not give any spatial information; therefore luminance contrast derived from spectral data between cuttlefish components alone cannot be used to assess the overall body pattern of a cuttlefish. However, it is a strong indicator of whether the body pattern is uniform or non-uniform.

While luminance contrast remained the same at depth for di- and trichromats in our analysis, color contrast $(\Delta \mathrm{S})$ showed some differences. The advantage of having a third visual pigment is best exemplified for the animal that does not have a good color match to the surrounding substrate (Figure 4.2b\&Figure $4.3 \mathrm{~b}$ ).

\section{The effect of water depth on color matching of camouflaged animals}

Broad-spectrum sunlight can be available under clear water at shallow depths on a cloudless day, especially in kelp or coral reef habitats. Under those conditions, colored objects (e.g., sand, rocks, algae, coral, tunicates, sponges, etc.) will appear colorful (Jerlov 1976; Chiao et al. 2000; Marshall et al. 2003a, b; Hochberg et al. 2004). However, many underwater light fields are not made up of broad-spectrum sunlight because light is scattered by particles suspended in water (e.g., plankton, sediment, algae, etc.) and attenuates non-uniformly with depth and wavelength (Tyler and Smith 1970). Therefore, many marine habitats do no appear particularly colorful, even if the substrate contains colorful objects. In shallow coastal areas, such as our dive site, water turbidity is key in limiting the spectral composition of daylight. At great depths, it is thought that camouflage by intensity matching is more effective than color matching, since the appearance of most objects become blue-green (see references in (Mäthger et al. 2006).At a depth of $10 \mathrm{~m}$, color contrast between the simulated animal spectra and substrate spectra was less substantial than differences between actual animal and substrate spectra collected at a depth of $1 \mathrm{~m}$. Luminance contrast, however, remained practically unchanged, as the ratios of quantum catches using attenuated spectra did not change significantly. This result suggests that the animal would have been less distinguishable from its background in terms of color had the spectra been measured at this depth (Figure 4.4), but would have appeared to have the same luminance contrast relative to the surrounding substrate despite attenuated ambient light. Mäthger et al. (2008) performed a similar simulation using laboratory data and showed that color match differences adjusted 
for a depth of $10 \mathrm{~m}$ were half as substantial as differences measured in a few centimeters of water.

To visualize this attenuation further, we used Maxwell triangles to represent colors from the visual system of a hypothetical trichromat predator (data only shown for one case, see Figure 4.6b). In the case of one animal that appeared to have a bad color match to its surroundings (Figure 4.2b\&Figure $4.3 \mathrm{~b}$ ) at $1 \mathrm{~m}$, the cuttlefish and substrate colors were relatively widely dispersed on the Maxwell triangles (Figure 4.6b). When the appearance of this animal was simulated at a depth of 10 meters, the loci of colors on the Maxwell triangle almost completely overlapped, indicating that this animal's body pattern would be better camouflaged in the eyes of trichomatic predators at a depth of 10 meters.

\section{Color matching in colorblind cuttlefish}

Researchers have been puzzled by the color-matching aspect of cuttlefish camouflage because cuttlefish are known to be colorblind (Brown and Brown 1958; Marshall and Messenger 1996; Mäthger et al. 2006). Mäthger et al. 2008 suggested that the spectral properties of $S$. officinalis body patterns and many natural objects are generally similar, thereby rendering color match less difficult. Our field data confirm their speculation for $S$. officinalis, at least for this particular study site. S. officinalis encounters a wide range of habitats including temperate rock reef environments throughout the Mediterranean and coral habitats off the west-central African coast. Certainly, some colors are not in the color repertoire of cuttlefish skin as Mäthger et al. (2008) showed in laboratory studies.

As cuttlefish approach sexual maturity, their skin undergoes a physiological and morphological change where iridophores and leucophores develop to form White zebra bands (Hanlon and Messenger 1988), an important component of body patterns used for sexual signaling. Although the White zebra bands can be masked by the overlying chromatophores, they are permanent and are often partially visible while a mature cuttlefish is camouflaged. One cuttlefish (Figure 4.2f) showed a weak zebra pattern and spectral measurements from individual light and dark bands had low luminance and color contrast relative to some of the surrounding substrates. This result supports the speculation of Hanlon and Messenger (1988) that, in addition to their role in signaling, the White zebra bands can contribute to camouflage when their bright contrast is modulated by actively masking them to varying degrees by overlying chromatophores. Overall, cuttlefish skin pattern components for camouflage closely resembled the luminance and color of surrounding substrates in the eyes of hypothetical di- and trichromatic fish predators we modeled, but the range of colors found in this particular habitat on both cuttlefish and substrates was narrow. Nevertheless, our light-field and animal/substrate measurements corroborate that the spectral properties of chromatophores and natural objects are similar, thus facilitating color matching by cuttlefish. Moreover, cuttlefish can neurally control the expression of chromatophores thus selectively reveal underlying reflector cell types such as leucophores, which also have some capability to tone match and perhaps reflect ambient wavelengths (Messenger 1974). Despite apparent colorblindness in cuttlefish, the tone and color matches between animal and background make cuttlefish camouflage superb in the animal kingdom (see selected images in (Hanlon et al. 2009). It remains a future challenge to discover how cephalopods achieve 
color resemblance to multiple backgrounds, and to what degree this is an active vs. passive process. This can be approached in the near future by testing the color matching abilities of cuttlefish in more chromatically diverse habitats (e.g., a coral reef) with hyper-spectral imagers and fuller characterization of the light field; currently, little is known about the color matching abilities of cuttlefish that live different habitats. Another approach, albeit logistically difficult, would be to transport cuttlefish native to a chromatically poor habitat to a chromatically rich habitat. Many such challenges remain in the field of sensory ecology, not just of cephalopods in marine habitats but many taxa involved in visual predator/prey camouflage interactions.

\subsection{Conclusion}

Although cuttlefish body pattern spectra were remarkably similar to many of the natural substrates at the Aegean study site, the color spectra of those particular background substrates and objects were limited (Fig. 4.4) and natural substrates that cuttlefish cannot match undoubtedly exist within its geographical range. For example, none of the parts of the animals in Figure $4.2 \mathrm{c} \&$ f closely matched the dark green Posedonia in color contrast while many of the measured body pattern components closely matched tan and brown sand and gravel substrates. From our study, it can be concluded that there is more to the discrimination of colors than simply a spectral shape mismatch. For example CIE $\Delta E_{2000}$ (Luo et al. 2001; Sharma et al. 2005) is a highly refined measure of how similar two colors appear to a human observer and contains weighting functions for lightness, hue and chroma and compensation factors for blue hue nonlinearity that previous CIE color difference formulations did not have. The correlation between SAM and $\Delta E_{2000}$ for the data we present here is much weaker $(|\mathrm{r}| \sim 0.3)$ than the correlation between SAM and $\Delta S$ (when this model is used with parameters that match those of the human visual system); this is not because SAM is better matched to animal visual systems but rather we know more about the human visual system and thus have derived a highly specialized color difference model. Regardless of how well an animal matches its background chromatically; its camouflage pattern becomes more effective at deeper depths due to the limited dynamic range, and uneven spectral attenuation underwater. 


\section{Chapter 5}

\section{Image-based quantification of cuttlefish (S.officinalis) camouflage patterns}

\subsection{Introduction}

The study of animal body patterning intertwines with many important questions in behavioral and evolutionary biology related to mate selection, communication, signaling and camouflage. For example, Hauglund et al. (Hauglund et al. 2006) found that the color and stripes of wasp patterns are visual signals that differentially affect the behavior of domestic chicks that hunt them; the yellow has an aversive effect on inexperienced predators while the stripes increase the speed of avoidance learning. While most animals have a pattern that undergoes little or no change throughout their lifetimes, cuttlefish are able to quickly change their appearance in response to visual stimuli (Hanlon and Messenger 1988; Hanlon and Messenger 1996; Messenger 2001a). In this work, we use image processing and computer vision tools to quantify cuttlefish camouflage patterns. We built an extensive database of calibrated photographs taken in the laboratory of camouflaged cuttlefish on 72 different backgrounds. Based on the images in our database, we asked the following questions: (1) What is the relationship between the levels of expression of chromatic body components? (2) Does the space of camouflage patterns form a continuum, or are there a discrete number of clusters? If so, how many? (3) Do cuttlefish express all possible patterns their components are capable of forming, or do they only show a subset?

Animal patterns are used for inter- and intra-specific communication, and for camouflage. Camouflage is an umbrella term that describes all strategies used for concealment, including body patterning and coloration, mimicking behavior, and motion (Stevens and Merilaita 2009). While there is no widespread consensus on the definitions of camouflage, Stevens and Merilaita make the distinction between function and strategy to describe them: i.e. the function of the evolutionary adaptation, such as breaking up form; as opposed to the strategy, the specific perceptual processes targeted, e.g. does the pattern disrupt normal edge detection mechanisms? In this work, we are most concerned with the crypsis function, which aims to initially prevent detection and includes strategies of background matching, distractive markings, disruptive coloration, self-shadow concealment, obliterative shading and flicker-fusion camouflage. Background matching works through blending in with the color, lightness or pattern of a background.

Distractive markings might stand out in a way that direct the attention of an observer away from the true shape of the animal, where disruptive coloration creates false edges and boundaries to hinder the detection or recognition of parts or the whole of an animal (Stevens and Merilaita 2009). Among so many possibilities, how can we determine which particular strategy an animal is employing? Stripes could disrupt an animal's outline, or could form distractive markings (Stevens and Merilaita 2009). How can we decide what their function is?

It has often been stated that our lack of knowledge of the perceptual mechanisms of the relevant observers hinders full understanding of camouflage strategies (Stevens and Merilaita 2009). An additional obstacle, which has received little attention in cuttlefish, is the lack of quantification of the limits to the cryptic coloration and patterns which can be 
produced by the animals. If the animal does not perfectly match the background, is this because it cannot, due to limits in its body patterning system? Or might the animal be implementing an alternative camouflage strategy, such as disruptive coloration? Camouflage only needs to deceive the visual system of the receiver - if there are limits to the patterns that can be produced, does that give us any insights about the vastly different visual systems of cuttlefish predators?

Cuttlefish skin exhibits great versatility through the display of attention-getting patterns for signaling, and highly cryptic patterns for camouflage. This makes it difficult to infer what kind of physiological limitations might exist amongst body components. For example, while the nervous control system of the cuttlefish allows for unilateral expression of patterns in a conspicuous appearance, we always observe a high degree of bilateral symmetry in camouflage patterns (Hanlon and Messenger 1988; Langridge 2006; Allen et al. 2010b); clearly, correlation between right and left side components is not a physiological limitation. If there were no limitations and all of the cuttlefish' body parts were independent of each other, cuttlefish could theoretically express every possible combination of pattern components. Based on our observations in the lab and in the field, some patterns are not common; a pattern with a White Square darker than all other components has not been reported. Curiously, based on our observations, cuttlefish can make very dark Uniform patterns, which means every component - including the White Square - can be made very dark. Why, then, does the White Square never go dark by itself? Understanding the limitations of components could lend insight into camouflage strategies, mechanisms, and physiology, as well as into the visual systems of their predators.

One of the most debated questions relating to cuttlefish camouflage is whether cuttlefish camouflage patterns produce a discrete set of patterns, as opposed to a continuum of patterns. If discrete classes, it becomes interesting to ask how many classes. A continuum of patterns may hint that cuttlefish might have only a single mechanism or strategy for producing camouflage patterns. On the other hand, if the animal sometimes implements a background matching strategy, and sometimes a strategy of producing distractive markings, then one might expect that the resulting patterns would appear to form well-defined and separated pattern classes. In that case, one could ask whether a given class serves a background matching or a disruptive coloration strategy.

These questions can be asked about the overall luminance changes produced by the larger scale body parts, and can also be asked of the small-to-medium scale "mottle" patterning. Is mottle patterning expressed at equal strength by each component or are some components not wired to show strong mottle spots? Is mottle expression correlated across the body of the cuttlefish? What are the limits to mottle spot sizes that can be produced by the cuttlefish skin?

In order to explore the gamut of body patterns, we must expose cuttlefish to a maximally diverse and unbiased collection of patterns. Collecting images of camouflage patterns is a time-consuming process, as time to realize a stable pattern ranges from a couple of minutes to sometimes an hour or more, and the number of trials each animal can be exposed to in a given day is limited to prevent stress to the animal. To observe as many unique patterns as possible, we surveyed the $S$. officinalis literature for background substrates used to evoke camouflage, and carried out an extensive project to build a 
database of raw (linear) images of cuttlefish camouflaged on these substrates, as well as additional ones designed de novo.

Consistent and controlled photographic data acquisition is essential for the success of this study, as it requires quantitative comparison of a large number of body patterns. We placed photographic calibration targets in each frame for dynamic range adjustment, and ensured all image intensities were scaled according to the same standard. We call the resulting set of images, composed of 5 animals placed on 72 different background combinations, the Cuttlefish $72 \times 5$ database.

Using the images in this database, we measured body component intensity and "mottleness", and derived a high-dimensional feature matrix. We found that the intensities of the head and body components are weakly negatively correlated, indicating that these parts may be used as two different elements of a pattern. In high-contrast patterns, the expression of the White Square and the White Triangle are highly correlated. We derived a map of physiological chromatophore units that control mottleness, and found that for the White Square and the White Triangle, both of which are on the axis of bilateral symmetry, have the least number and total area of dark spots, suggesting that one of their functions might be to create a visual low-frequency barrier, disrupting the animal's shape. Our findings support the qualitative statement made by Chiao et al (2010) that there are two sizes of spots cuttlefish can produce mottleness with, and that the areas of small light and dark mottle spots are approximately equal in every pattern they are expressed. We found that there was no optimal number of clusters that represented the patterns in our database. Finally, based on principal components analysis of the images in our database, cuttlefish show a small subset of the body patterns they could theoretically display, if their components all acted independently. 


\subsection{Background and related work}

\section{Brief summary of animal pattern quantification techniques}

In this section, we provide a brief overview of commonly used animal pattern quantification techniques, and then describe those used for cuttlefish patterns in detail. For cuttlefish, the quantitative relationship between the levels of expression of body components, and their capabilities, has not previously been studied. Addressing this question is important because understanding the relationships between components is key to evaluating the space of all possible patterns that can be shown. Numerous studies have investigated, qualitatively and quantitatively, what the total number of camouflage patterns available to cuttlefish might be (e.g., Hanlon and Messenger 1988; Crook et al. 2002; Anderson et al. 2003). However, most of these studies suffer from two principal shortcomings. First, it often includes steps of scoring done by humans. While manual scoring is quick and does not require any specialized instruments, it is inherently subjective and assigned grades often vary between observers, causing low repeatability (Stevens 2011). Second, in some cases patterns from different photographs are warped onto a template so certain features can be compared locally when analyzing multiple images of the same animal (Shohet et al. 2007; Anderson et al. 2003; Barbosa et al. 2008b). This step is error-prone and might produce blurring and aliasing artifacts compromising data quality (Szeliski 2010); such problems have already been reported in the context of cuttlefish patterning (Shohet et al. 2007; Anderson et al. 2003). But more importantly, these studies assume that well-defined, discrete clusters of body patterns exist and attempt to determine their number. Here, we will question the validity of this assumption and take existing work further through the use of methodologies that investigate the structure of the space of camouflage patterns, while ensuring that subjective human input, data acquisition and post-processing artifacts are minimized.

Allen and Higham (Allen and Higham 2013) describe animal patterns as the parts of skin, fur or plumage visible to others, containing spatial variations of color and structure, forming spots, stripes, blotches and gradients, often with repetitions. Before the widespread use of computers and digital images, patterns and backgrounds were often analyzed by taking 1-D transects across (digitized) images and deriving parameters describing color, intensity or pattern across these transects. This process is still frequently used (e.g., for water snakes (King 1992); bowerbird ornaments (Endler and Day 2006); and for cuttlefish component intensity (Chiao et al. 2009)), and is detailed in (Endler 1984)). With the advent of faster computers, researchers have adopted frequency domain methods for the analysis of 2-D patterns. These methods assess how much information patterns carry at various spatial scales and orientations e.g., tiger and zebra stripes (Godfrey et al. 1987); cuttlefish patterns (Shohet et al. 2006; Barbosa et al. 2008b; Chiao et al. 2009; Zylinski et al. 2011); and avian eggs (Stoddard and Stevens 2011; Spottiswoode and Stevens 2012), and are well suited to most patterns. The biggest drawback of frequency-domain methods is that spatial information is discarded, which is important for many research questions. For example, detecting only the energy carried by mottle spots in cuttlefish patterns and discarding their location prevents the understanding of whether their expression is correlated among components, or how it varies between patterns. 


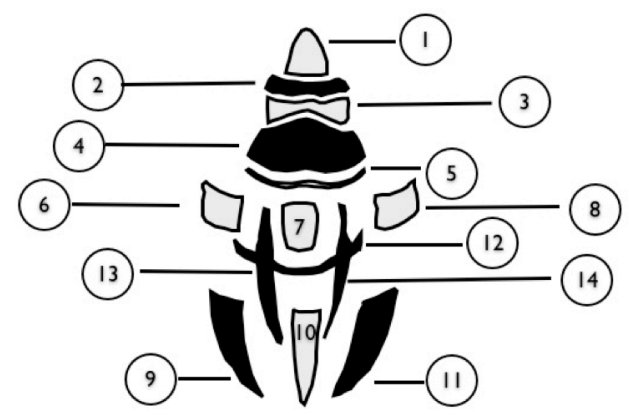

\begin{tabular}{|c|c|c|}
\hline Component Name & $\begin{array}{c}\text { Our } \\
\text { numbering } \\
\text { convention }\end{array}$ & $\begin{array}{c}\text { Hanlon \& } \\
\text { Messenger } \\
1988 \\
\text { convention }\end{array}$ \\
\hline White arm triangle & 1 & 14 \\
\hline Anterior head bar & 2 & 29 \\
\hline White head bar & 3 & 13 \\
\hline Anterior mantle bar & 4 & 19 \\
\hline $\begin{array}{c}\text { Anterior transverse } \\
\text { mantle line }\end{array}$ & 5 & 17 \\
\hline White mantle bar & 6 & 3, Left \\
\hline White square & 7 & 2 \\
\hline White mantle bar & 8 & 3, Right \\
\hline Posterior mantle bar & 9 & 20, Left \\
\hline $\begin{array}{c}\text { White posterior } \\
\text { triangle }\end{array}$ & 10 & 1 \\
\hline Posterior mantle bar & 11 & 20, Right \\
\hline $\begin{array}{c}\text { Posterior transverse } \\
\text { mantle line }\end{array}$ & 12 & 18 \\
\hline Median mantle stripe & 13 & 22, Left \\
\hline Median mantle stripe & 14 & 22, Right \\
\hline
\end{tabular}

Figure 5.1 We number components differently than the convention used by Hanlon and Messenger (Hanlon and Messenger 1988), in which Sepia officinalis body patterns and their components were first described. We do not consider some of the small chromatic components (e.g., White Fin Spots, White Fin Line, Pink Iridophore Arm Stripes etc.) and therefore a top-down, left-to-right numbering convention utilizing consequent numbers allows for more intuitive interpretation of results. The light $(1,3,6-8,10)$ and dark $(2,4,5,9,11-14)$ chromatic components, as described in (Hanlon and Messenger 1988) are color coded here for completeness. Throughout the text, we will prefix components with "C". Unless noted otherwise, $\mathrm{C6} \& \mathrm{C8}, \mathrm{C} 9 \& \mathrm{C} 11$ and $\mathrm{C} 13 \& \mathrm{C} 14$ will be assumed to exhibit bilateral symmetry and only one of each pair will be shown in results.

\section{Existing body of work on cuttlefish patterns}

There has been significant previous work describing cuttlefish patterns and coloration, yet much of that work has focused on different questions such as the effectiveness of camouflage in the eyes of predators (e.g., (Akkaynak et al. 2013; Chiao et al. 2011; Zylinski et al. 2009c; Mäthger et al. 2008; Hanlon et al. 2013) and the identification of camouflage strategy facilitated by the pattern shown (e.g., (Zylinski et al. 2012; Buresch et al. 2011; Allen et al. 2010b; Allen et al. 2010a; Barbosa et al. 2008b; Kelman et al. 2008; Barbosa et al. 2008a; Kelman et al. 2007; Shohet et al. 2006; Mäthger et al. 2006; Chiao et al. 2005; Grable et al. 2002), or determining which elements in a given background influence final pattern expressed (e.g., (Ulmer et al. 2013; Chiao et al. 2013; Hanlon et al. 2013; Chiao et al. 2010; Lee et al. 2010; Hanlon et al. 2009; Zylinski et al. 2009c, a; Barbosa et al. 2008b; Mäthger et al. 2007; Barbosa et al. 2007; Kelman et al. 2007; Chiao et al. 2007; Chiao et al. 2005; Grable et al. 2002; Chiao and Hanlon 2001a, b; Marshall and Messenger 1996).

Previous studies most relevant to our research questions seek to identify quantifiable differences between patterns, and determine the number of existing camouflage patterns. Hanlon and colleagues (Hanlon and Messenger 1988) were the first to describe 13 overall body patterns, 5 of which were used for camouflage, based on visual observations in the lab and in the sea. The five camouflage patterns are generally sorted into three broad 
classes called Uniform (U), Mottle (M) and Disruptive (D), allowing some variation within each. These three classes differ from each other in contrast and granularity (spatial scale): Uniform patterns have low contrast and fine spatial scale, whereas Mottle patterns have coarser elements of moderate contrast, and Disruptive patterns have large elements of high contrast.

\section{Contrast quantification}

Chiao and colleagues introduced a manual scoring system that assessed the level of expression of each chromatic body component (Chiao and Hanlon 2001a, b), to describe a body pattern. Further developed by Mäthger and colleagues (Mäthger et al. 2006), this method is known as disruptive scoring. The term component expression in the context of disruptive scoring requires some clarification. Chromatic components are divided into two classes: dark and light as defined in (Hanlon and Messenger 1988). Dark components on the dorsal side are produced by expanded chromatophores (Figure 5.1, components shown in black, 2,4-5,9,11,12-14), while light components result from retracted chromatophores revealing underlying leucophores, translucent skin or the cuttlebone (Figure 5.1, components shown in light gray, components 1, 3, 6-8, 10). Generally (somewhat confusingly), for disruptive scoring, a light chromatic component is considered expressed when it is brighter than a threshold, and a dark component is considered expressed when it is darker than a threshold. Often, this threshold is chosen to be the mean intensity of the pattern. When all components are minimally expressed (score of 0 ), the body pattern is Uniform; when all are maximally expressed (score of 3 ), the body pattern is the Disruptive pattern as described in (Hanlon and Messenger 1988). Mathger et al used only 11 components for scoring, yielding a maximum score of 11 x 3 $=33$ when light components were maximally light, dark components were maximally dark (Mäthger et al. 2006). This method was automated partially by Chiao et al. (Chiao et al. 2009), by warping the cuttlefish image to a standard template through user-marked correspondence points and summing Weber contrast of components relative to the image mean, along transects. Chiao and colleagues found that semi-automatic disruptive scores predicted human assigned scores well $\left(\rho^{2}=0.9683\right)$, suggesting that this method could effectively replace human observers (Chiao et al. 2009).

\section{Mottleness quantification}

Uniform, Mottle and Disruptive pattern types differ in contrast and the spatial scale of their mottle spots; and while the disruptive score captures contrast differences quite well, it does not describe differences in spatial scale. To quantify elements that vary in spatial scale, or mottleness, Barbosa et al. introduced granularity analysis (Barbosa et al. 2008b). This method filters cropped and warped cuttlefish images using 6 octave-wide bandpass filters and retains the sum of the squared filter outputs (i.e., energy) for each filter. Two parameters then describe the pattern: total energy (TE), which is an indicator of overall contrast, and mean granularity (MG), which measures the average spatial frequency of the body pattern (Chiao et al. 2009). 


\section{Other methodology}

Zylinski et al. (Zylinski et al. 2009a) used principal components analysis (PCA) on the manually graded expression of body components (plus, additional features such as mottle spots) and equated principal components to body patterns to quantify patterns cuttlefish showed during motion $v s$. when stationary. They found that motion patterns were lower contrast than static patterns. Investigating the total number of cuttlefish body patterns, Crook et al. (Crook et al. 2002) used AutoClass, (Cheeseman et al. 1993) a Bayesian clustering algorithm, and found that $12-14$ body patterns were most likely. They used a generative model approach to propose a large set of models that might explain how the body patterns were generated from components, and then used Bayesian statistics to estimate the posterior probability of these signals having been generated by a particular model. In addition to the 34 chromatic components identified for $S$. officinalis in (Hanlon and Messenger 1988), they included six textural, eight postural and six locomotor components (total of 54 components, $\mathrm{N}=808$ photographs). The inputs to the AutoClass algorithm were the human assigned scores of the level of expression of each of the 54 components. While their findings agreed in quantity with the 13 patterns identified in (Hanlon and Messenger 1988), their study made no judgment about which or how many of these might be camouflage patterns because they analyzed behaviors that included feeding, foraging and social communication in addition to camouflage. Using photographs of cuttlefish hunting and eating as well as of camouflaged

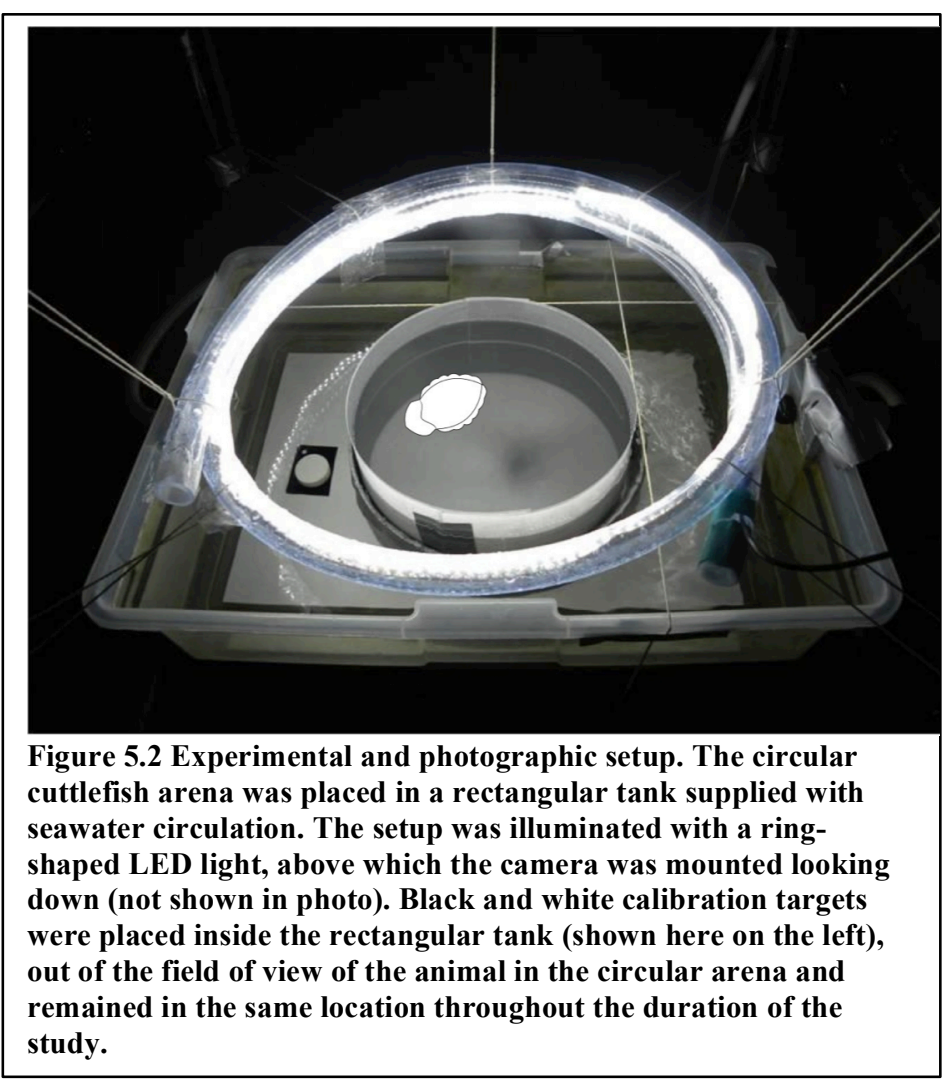
individuals, Anderson and colleagues (Anderson et al. 2003) identified three temporal and three spatial components, but the small sample size ( 30 cuttlefish photographs) limits the effectiveness of their study.

Next, we describe the construction of the Cuttlefish $72 \times 5$ database.

\subsection{Experimental Setup}

Animals Five adult (mantle length: 6.2-7.2 cm) European cuttlefish (Sepia officinalis) hatched, reared and maintained at the MBL Marine Resources Center (Woods Hole, MA, USA) were used for the experiments. Animals were housed separately for the duration of the study. 
Experimental setup Each test animal was placed in a circular arena, $(23 \mathrm{~cm}$ in diameter, walls $14 \mathrm{~cm}$ high), inside a rectangular tank $(55 \times 40 \times 15 \mathrm{~cm})$ with flow-through seawater (Figure 5.2). Experiments ran over a period of three months (September-December 2012), during which animals performed one trial for every different substrate, and no more than two trials in a single day to minimize any stress on animals. To avoid disturbance to the animals and control lighting, the experiments were conducted inside a closed tent of black sheeting and the test animal was observed using an external video monitor. Each individual was given a minimum of 20 minutes to settle on the substrate; thereafter, trials were ended when the animal was seen to have maintained a stable position and pattern for a period of 2 minutes.

Photographic setup Black (Digital Kolor Kard) and white (Spectralon; Labsphere, NH) photographic calibration targets, which remained in the same position throughout the entire study, were placed in the experimental setup in order to allow for careful calibration of the dynamic range in each photograph (Figure 5.2). These targets were not visible to the animals. To reduce shadows cast inside the arena and minimize lighting variation, the tank was illuminated by a ring of LED lights ( $40 \mathrm{~cm}$ diameter), suspended 5 $\mathrm{cm}$ above the tank. Photographs were taken every 10s for the duration of each trial with a Canon EOS $1000 \mathrm{D}$, fixed $50 \mathrm{~cm}$ above the tank and triggered remotely by a TimeMachine (Mumford Micro Systems) device. The tank, lights and camera remained in a constant position throughout the experimental period.

Substrates Substrates presented to the animals on the floor and walls of the arena were chosen to capture the range of backgrounds previously tested in psychophysics experiments performed with cuttlefish in both our laboratory and others (Figure 5.3). To elicit a variety of camouflage body patterns, we also designed novel substrates to test specific characteristics of the cuttlefish visual system. In total, we used 50 substrates, natural and artificial, two-dimensional and textured, which were presented to the animals in random order. In experiments with natural or artificial substrates, as well as with photographs of natural substrates, the walls were uniform grey 50\% (RGB 122), while computer-generated backgrounds were paired with both matching and uniform grey walls. As a result, 72 different background pattern combinations were tested. Substrate order was randomized, but remained the same for each of the five individuals. 


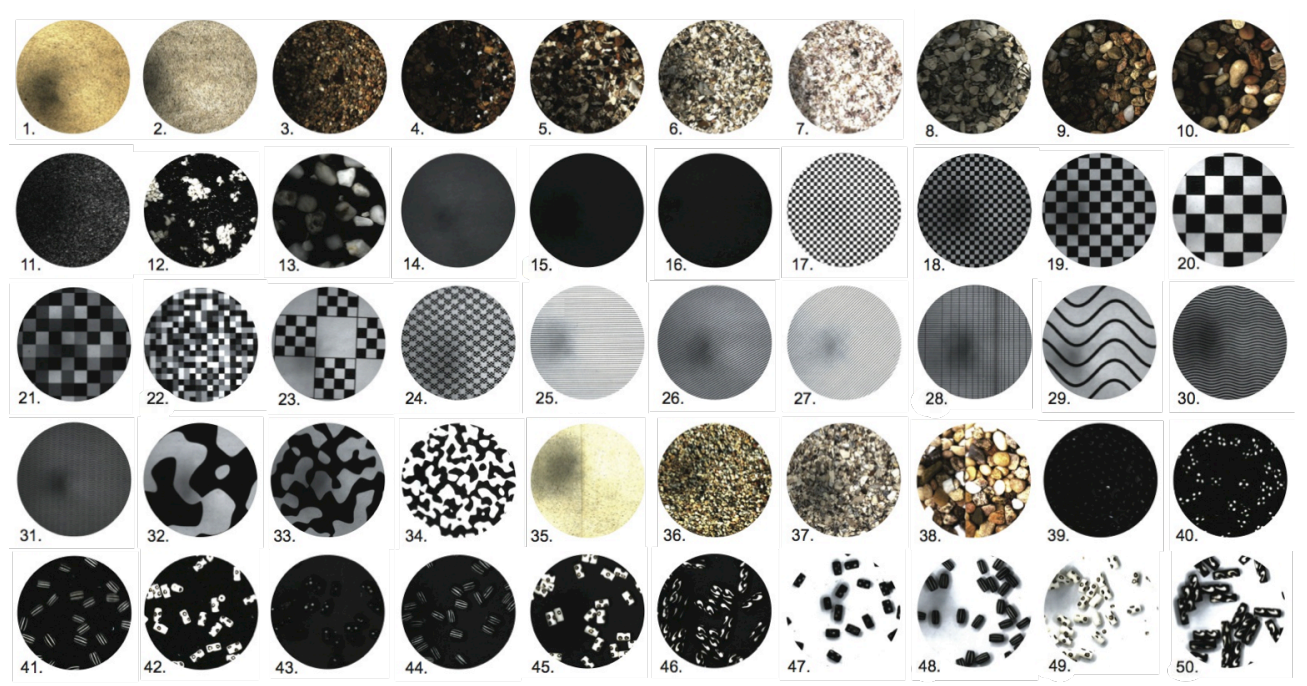

Figure 5.3 Stimuli from existing studies (e.g., (Hanlon et al. 2013; Chiao et al. 2010; Lee et al. 2010; Hanlon et al. 2009; Zylinski et al. 2009c, a; Chiao et al. 2009; Barbosa et al. 2008b; Mäthger et al. 2007; Barbosa et al. 2007; Kelman et al. 2007; Chiao et al. 2007; Chiao et al. 2005; Grable et al. 2002; Chiao and Hanlon 2001a, b; Marshall and Messenger 1996; Shohet et al. 2007)) form the basis of the images in the Cuttlefish 72x5 database 1 - Light brown sand, 2- White sand, 3- Gravel, 4-Dark gravel, 5-Shell bits dark,6-Shell bits light, 7-Pink coralline, 8-Gray shells, 9-Pebbles, 10-Brown pebbles, 11Black and white sand, 12-Black and white gravel, 13-Black and white pebbles, 14-Uniform gray RGB 122, 15-Uniform dark gray, RGB 61, 16-Black RGB 0, 17-Checkerboard 4mm, 18-Checkerboard 5mm, 19-Checkerboard 12 mm, 20-Checkerboard 20mm, 21-Scramble P1 5x5 (1.27 mm), 22-Scramble P1 5x5 (5.5mm), 23-Tiled checks 10mm, 24- Tiled checks $(5 \times 7 \mathrm{~mm})$, 25-Horizontal stripes, 26-Vertical $30^{\circ}$ stripes, $27-V e r t i c a l ~ 45^{\circ}$ stripes, 28 -Frequency sweep, 29 -Sine wave 1 , 30-Sine wave 2,31 -Sine wave 3, 32-Cow BP72_pt5, 33-Cow BP36_pt5, 34-Cow BP18_pt5, 35-Photograph of sand, 36Photograph of gravel, 37-Photograph of gray shells, 38-Photograph of pebbles, 39-Round black beads, uniform black background, 40-Round black beads, black and white spotted beads, uniform black background, 41-Round black beads, black and white striped beads, uniform black background, 42-Round black beads, white and gold beads, uniform black background, 43-Black and white spotted beads, uniform black background, 44-Black and white striped beads, uniform black background, 45White and gold beads, uniform black background, 46-Black and white large beads, uniform black background, 47-Black and white spotted beads, uniform white background, 48-Black and white striped beads, uniform white background,49-White and gold beads, uniform white background, 50Black and white large beads, uniform white background.

Image pre-processing Camera raw images $(I)$ were processed using black (B) and white (W) calibration targets to obtain the white balanced image $I_{i}^{\mathrm{WB}}$ as follows:

$$
I_{i}^{\mathrm{WB}}=\frac{I_{i}-B_{i}}{W_{i}-B_{i}} \quad \mathrm{i}=\mathrm{R}, \mathrm{G}, \mathrm{B}
$$

White balanced RGB images were then converted to the LAB color space (Wyszecki and Stiles 2000), and only the L (luminance) channel was retained. Since all images were captured with the same sensor, the analysis was done in the camera color space and no color transformation was applied (see (Akkaynak et al. 2014). The intensity images were then presented to an experienced user (DA) through custom-made software written in MATLAB (Mathworks, Inc., Natick MA) language, who marked the orientation the 
cuttlefish and labeled the body pattern components. Component-level labeling was done based on the definition of chromatic components in (Hanlon and Messenger 1988) but components were numbered differently (Figure 5.1). To speed up processing for certain tasks, images were automatically registered based on the intersecting reference strings in the lower left corner of each frame (Figure 5.2). With the user-made masks, average intensity for each component in the L image was calculated, forming a $360 \times 14$ intensity matrix for all the images in the Cuttlefish $72 \times 5$ database.

Shadow removal Even though we designed our photographic setup to minimize shadows cast over the arena, cuttlefish showed high preference for settling near arena walls. In those instances, one side of the cuttlefish was darker than the other in the captured photographs (Figure 5.4a). We remedied this situation by taking advantage of cryptic pattern symmetry (Langridge 2006), and matching the smoothed pixel intensity histogram of the shadowed half to that of the unshadowed half. This operation resulted in a visible seam in the middle (Figure 5.3c), which we concealed by blending the two image halves at multiple sub-bands using Laplacian pyramids combined with spatial feathering (Figure 5.4d) (Burt and Adelson 1983). While this approach only approximates intensity values for the shadowed pixels, it maintains local contrast, allowing us to find local extrema.

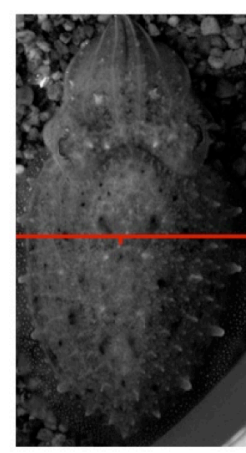

(a)

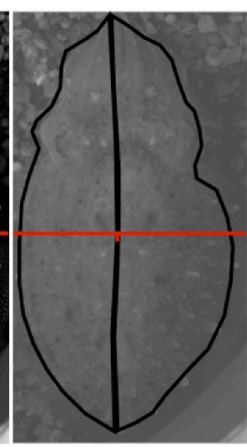

(b)

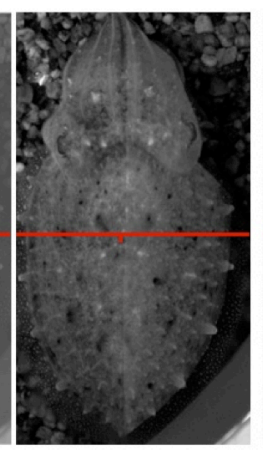

(c)

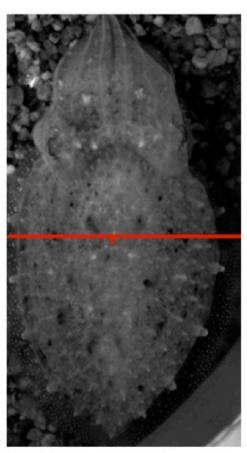

(d)

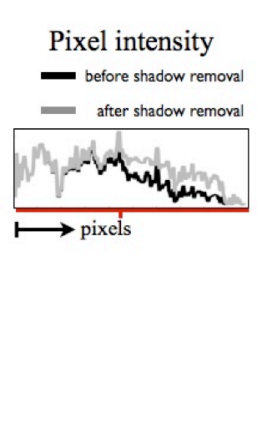

(e)

Figure 5.4 Example of shadow removal results. For each original image, the histogram of the shadowed lateral half was adjusted to match that of the unshadowed half, which was repeated at four different scales. (a) Original image (b) User-made right and left-half masks. The shadow removal operation left a visible seam between the two halves (c), which was blended through feathering (d). (e) Intensities of pixels along a horizontal transect across the image (red line) before and after shadow removal.

Next, we investigate our three main research questions using the imagery in the Cuttlefish $72 \times 5$ database. 


\subsection{What is the relationship between the levels of expression of chromatic components?}

The appearance of a cuttlefish's body pattern is a combination of three different kinds of components: chromatic, textural, postural and locomotor; which can be recombined at any time to make a new pattern (Hanlon and Messenger 1996). Components are made of units (e.g., morphological organization of cells in the skin into a circular patch), which are in turn composed of elements (e.g., chromatophores, idirophores, etc.). The physiological hierarchy between the elements, units and components of a cephalopod's body pattern is well established (Packard and Hochberg 1977; Hanlon and Messenger 1988). Generally parallel to this physiological hierarchy is a neural hierarchy, in which body patterns are organized at higher levels in the brain (i.e., the optic lobes), and chromatophores in the lower motor centers (Hanlon and Messenger 1996). Here, we are interested in the functional hierarchy between chromatic components: Does the expression of one component tend to trigger the expression of other(s)? Is the appearance of component A correlated with that of component B? Is component A only expressed above a certain threshold when component B is? Identifying such relationships will enable accurate quantification of body patterns, which in turn will help answer more complex questions like 'How many body patterns are there?" and give us insights into what aspects of predator vision the expression of a particular combination of components might deceive.

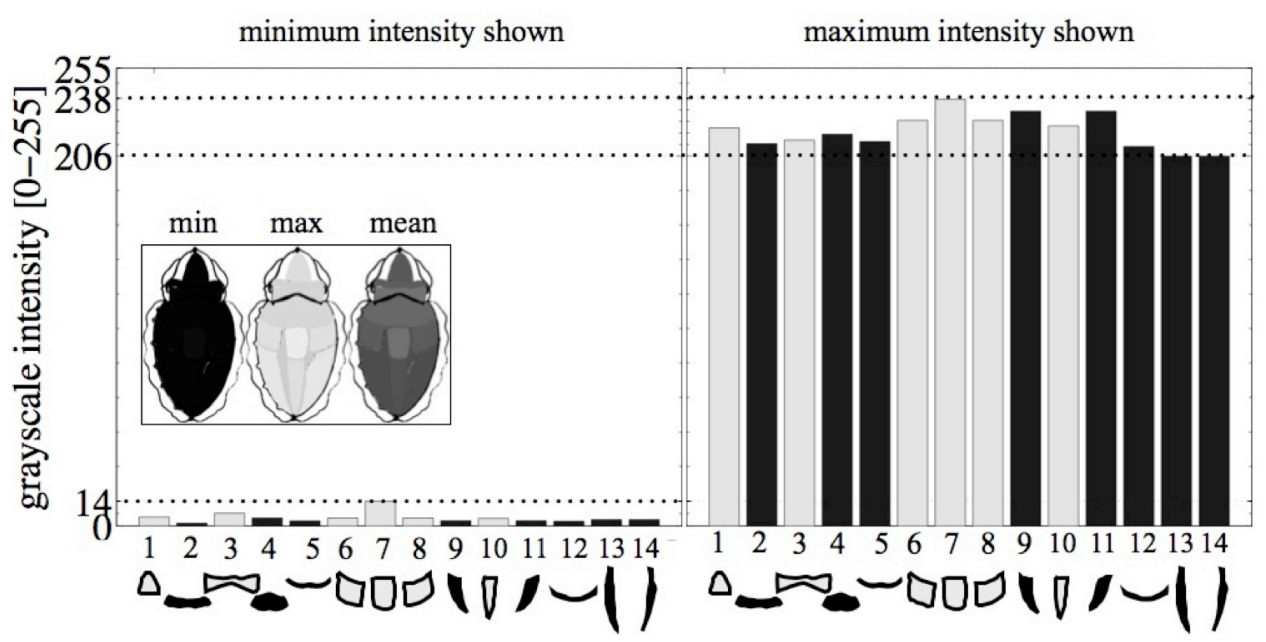

Figure 5.5 Minimum and maximum intensities shown by each component in the Cuttlefish $72 x 5$ database. (Note: we only present results for one of symmetric component pairs $6 \& 8,9 \& 11$ and $13 \& 14$, because of imposed bilateral symmetry during the shadow removal process.) All components showed a similar range of intensities, within the imposed dynamic range limits of 0 and 255. The White Square (component 7) has lighter minimum and maximum values compared to other components. Dashed lines indicate the upper and lower values of intensities each component showed; the darkest maximum intensity shown had a value of 206 (on a scale of 0 255), while the lightest was 238 . The lightest minimum intensity shown was 14 , and the darkest was 3 (not labeled). Inset: visualization of the minimum, maximum and mean grayscale intensity of each component. 


\section{Intensity}

Figure 5 shows the minimum and maximum intensities displayed by each component across the 360 images in our database. Even though components are historically classified as "light" and "dark", every component expressed a similar range of intensities. The White Square (C7) was the only (though modest) exception; its extrema were lighter than those for all other components. A principal components analysis done on all the images in our database reveals that the first principal component of body part intensities is the average pattern luminance, which explains over $87 \%$ of the variance. This is not surprising, because cuttlefish can show the same pattern (e.g., a dark Uniform and a light Uniform) with varying levels of intensity (Buresch et al, under review). Thus, to observe the other interesting aspects of component relationships more clearly, we subtracted the mean luminance from each image to obtain zero-mean image $\boldsymbol{I}_{z m}=\left(\boldsymbol{I}_{\text {int }}-\mu\right) / \mu$, where $\boldsymbol{I}_{\boldsymbol{i n t}}$ is the 14-long pattern intensity vector for a given pattern, and $\mu$ is its mean intensity. The distribution of zero-mean intensities for each component is given in Figure 5.6.

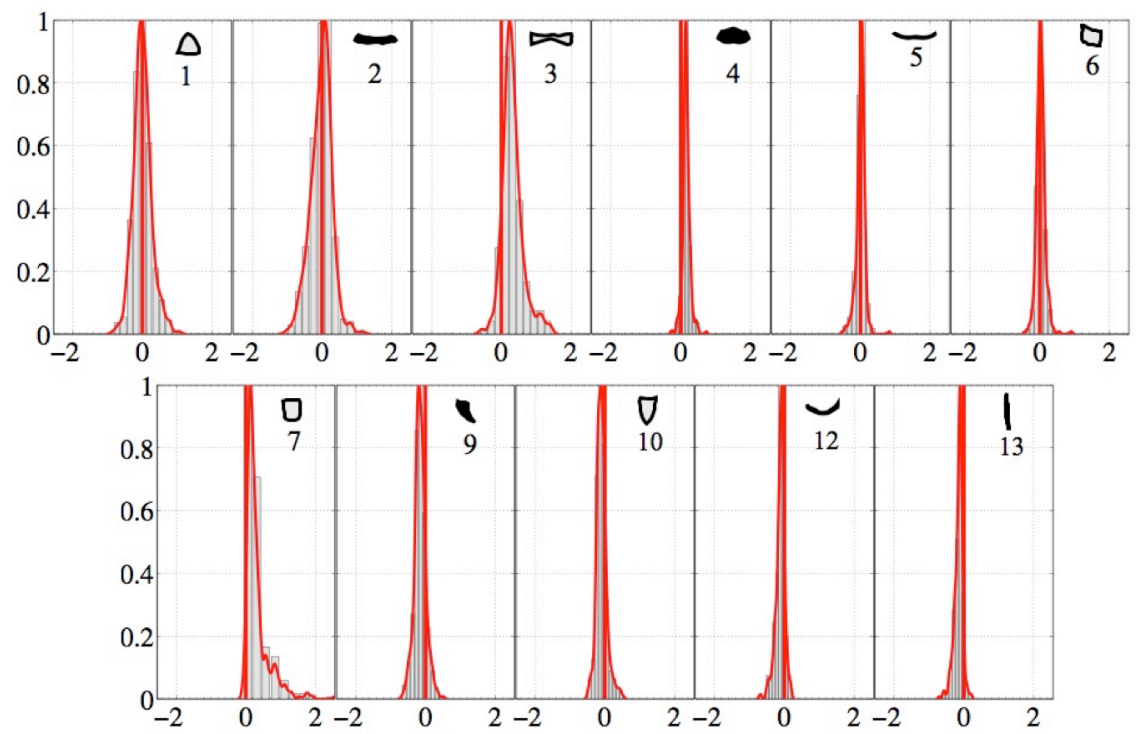

Figure 5.6 Distribution of zero-mean intensities per component; red curve shows the kernel density estimate; vertical red line shows $x=0$. While every component has taken on values brighter and darker than the pattern's overall mean intensity, the White Square (C7) has overwhelmingly taken lighter values. In other words, the White Square was almost never expressed darker than the mean intensity of the pattern $(p(C 7<0) \leq 0.027)$ based on the cumulative density function of its kernel density estimate. For other light components these probabilities were: $p(C 1<0) \leq$ $0.4854, p(C 3<0) \leq 0.1014, p(C 6<0) \leq 0.4079, p(C 10<0) \leq 0.791$; and for dark components: $p(C 2>0) \leq 0.5146, p(C 4>0) \geq 0.9730, p(C 5>0) \geq$ $0.6118, p(C 9>0) \leq 0.1749, p(C 12>0) \leq 0.1684$ and $p(C 13>0) \leq 0.0632$. Note that the components on the head (1-3) showed a wider range of relative intensities compared to all other components, which was significant $(F=0.75-4.7199$, p < 0.05).

To get an understanding of component relationships, we first look at pairwise correlations between their zero-mean intensities (Figure 5.7a). We observe the strongest positive correlation between components $12 \& 13$, which are the thin, linear, dark chromatic components on the body. This indicates that when they are expressed, they 
have roughly the same intensity, perhaps either blending in as a part of a low-contrast pattern, or standing out as linear structures in a high-contrast pattern. The strongest negative correlations are observed between components $2 \& 10$ and $3 \& 9$. These are opposite polarity (i.e., light/dark) pairs and the negative correlation between them indicates that they are used as contrast-building elements. Components on the head (1-3) are positively correlated with each other, but generally negatively correlated, or uncorrelated, with the other components on the body (6-13). The expression of the head and mantle components at contrasting mean intensities might suggest the simultaneous use of two different strategies; through one body part blending in with the background and other standing out, the animal's shape might be disrupted. We have observed patterns with head/mantle component contrast in our database (Figure 5.7b), this result may be partially attributable to the illumination differences across the experimental arena (Figure $5.7 \mathrm{c}$ ) because cuttlefish often positioned themselves at a right angle to the arena walls, which might have cast a laterally uneven shadow on them. Based on photographs alone, we cannot determine whether cuttlefish actually detected these shadows and intentionally displayed contrasting intensities between their heads/bodies to match the varying light pattern, or whether the head and mantle simply registered different pixel intensities in the photographs due to shadows.

Figure 5.7 combines data from all observed patterns. Including a large number of patterns which are nearly uniform may obscure interesting relationships present in the higher-contrast patterns. In Fig. 8, we focus only on high contrast patterns. We select high contrast patterns as those in or above the $90^{\text {th }}$ percentile of $\sum_{i=1}^{14} \sigma\left(\boldsymbol{I}_{z m}\right)$ for each pattern where $\sigma$ is the standard deviation, and $i$ is the index of each of the 14 components; this yields a total of 36 patterns. In this subset of patterns, components $1 \& 2$ and $7 \& 10$ showed the strongest positive correlation; and $\mathrm{C} 12 \& \mathrm{C} 13$ remain moderately positively correlated. Components $1 \& 2$ are commonly labeled as having opposite polarity (light expressed vs. dark expressed, respectively), but they can show intensities ranging from light to dark (Figure 5.6); and amongst the high contrast patterns in our database, they showed similar, not contrasting intensities. A newly emerged high correlation between C7\&10 is more interesting because while $\mathrm{C} 10$ showed both light and dark intensities (Figure 5.6), C7 overwhelmingly showed intensities lighter than the pattern mean. Thus, the high correlation between $\mathrm{C} 7 \& 10$ indicates that in high contrast patterns, $\mathrm{C} 10$ is exclusively expressed at mean pattern intensity or lighter, making it a key element for contrast. Component 10 expression is also strongly negatively correlated with all head components (1-3), indicating that often, but not always, a high-contrast pattern has a dark head. The lack of very strong correlations amongst components indicates that there is not a single high-contrast pattern and components can combine in different ways to make many such patterns; four examples are shown in Figure 5.8b.

While looking at component correlations is informative, it is difficult to infer what the relationships between more than two components may be from pairwise plots. To remedy that, we used a dendrogram to visualize the same data from Figure 5.7\&Figure 5.8 in a hierarchical layout. In Figure 5.9a, we see three main clusters; components on the head (1-3) along with the mantle component nearest to the head (4, cluster1); thin, linear, dark components on the body $(5,12,13 \& 14$, cluster 2$)$; and the remaining, larger components on the body (6-11, cluster3). Cluster1 appears to be weakly negatively correlated with clusters $2 \& 3$; which are uncorrelated with each other; these relationships parallel the 
observations we made for pairwise correlations in Figure 5.7. The same three clusters remain in the high-contrast subset of patterns (Figure 5.9b) with one important difference in cluster3: C7 becomes less correlated with all the other components in this cluster. This is likely because the White Square may or may not be fully expressed in a high contrast pattern (Figure 5.7b). Component 10 has a positive correlation with its neighboring components 9\&11; this behavior can be seen in all of the examples given in Figure 5.7b. 

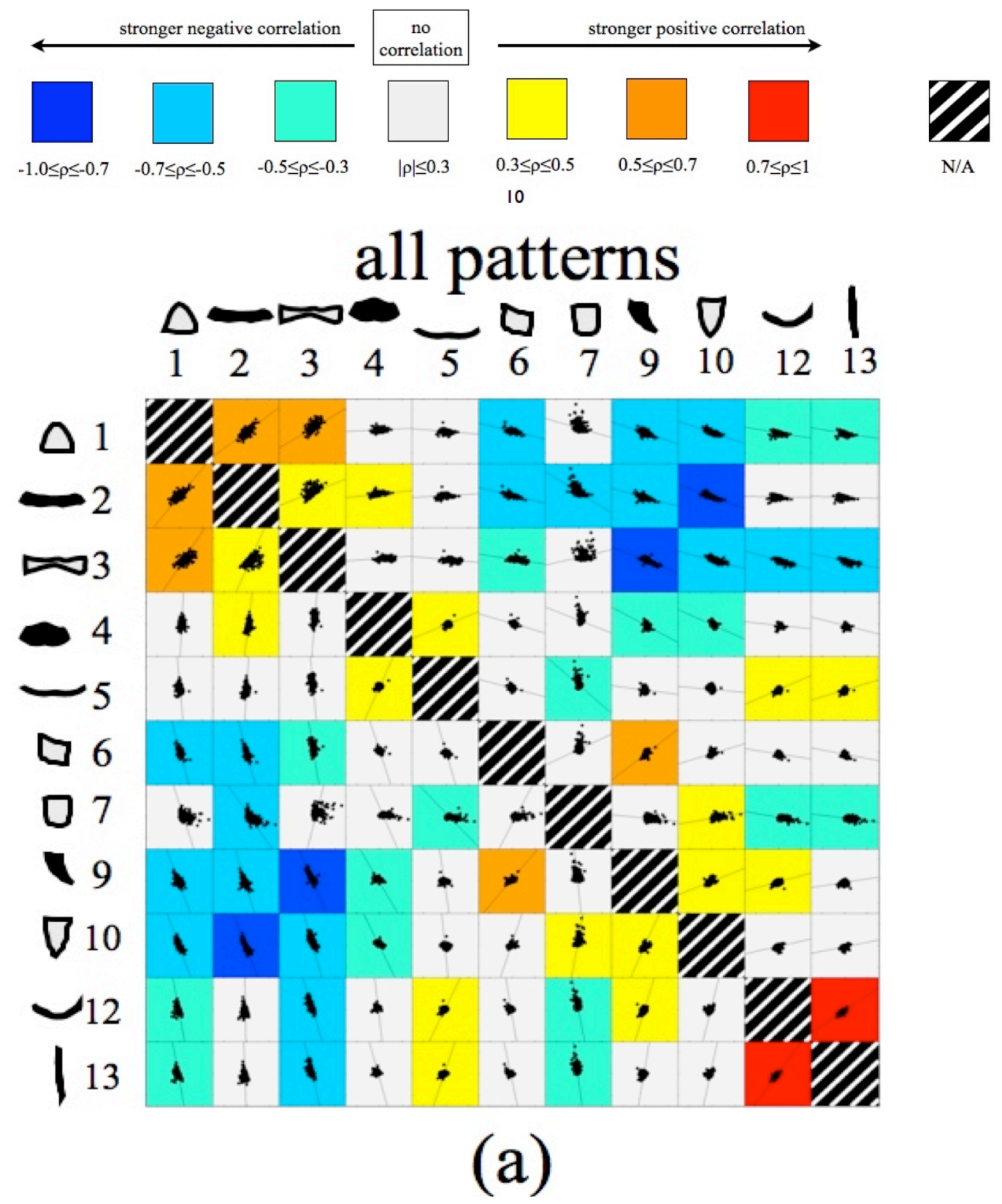

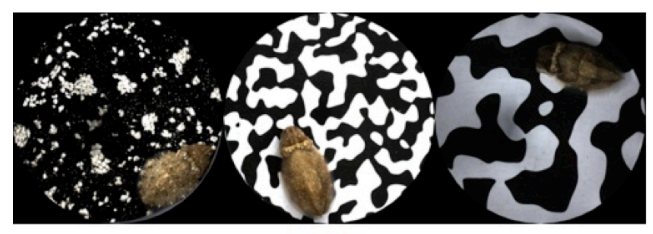

(b)

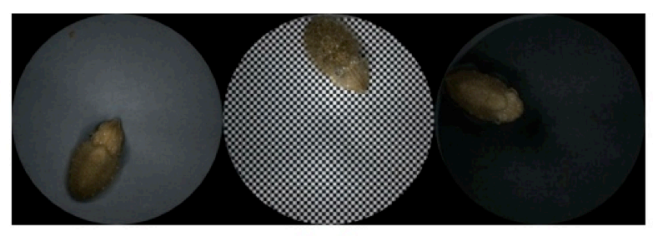

(c)

Figure 5.7 Here we show the pairwise correlations of zero-mean intensities between components for all patterns in the Cuttlefish $72 x 5$ database. Axes of each plot have been adjusted to [-3,3]. (b)

Examples of photographs with contrasting head/body pattern intensities. (c) Examples of photographs where the recorded head/body intensity difference is likely due to shadowing from arena walls. 

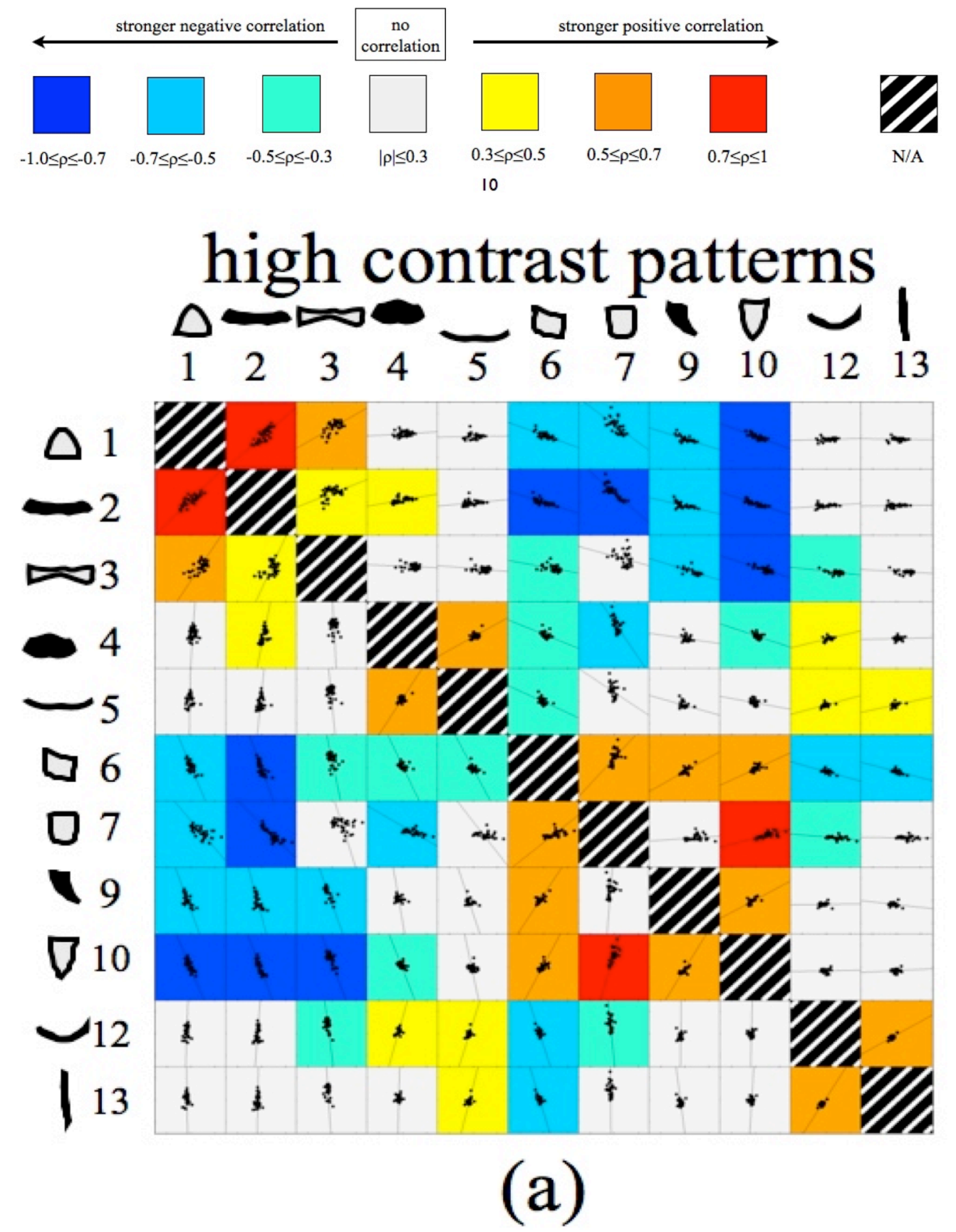

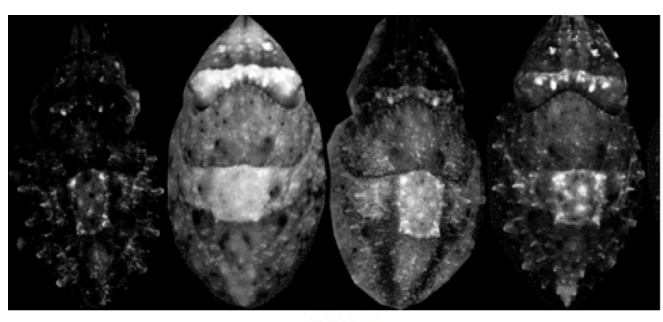

(b)

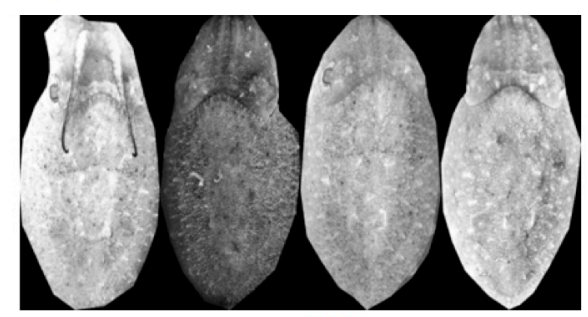

(c)

Figure 5.8 (a) Pairwise correlations of zero-mean intensity between components for 36 highcontrast patterns, that were selected as those in the $90^{\text {th }}$ percentile of $\sum_{i=1}^{14} \sigma\left(I_{z m}\right)$ for each pattern where $\sigma$ is the standard deviation, and $i$ is the index of each of the 14 components. Low contrast patterns were selected as those in the $10^{\text {th }}$ percentile or less. Axes of each plot have been adjusted to $[-3,3]$. (b) Examples of high and (c) low contrast patterns from our database. 


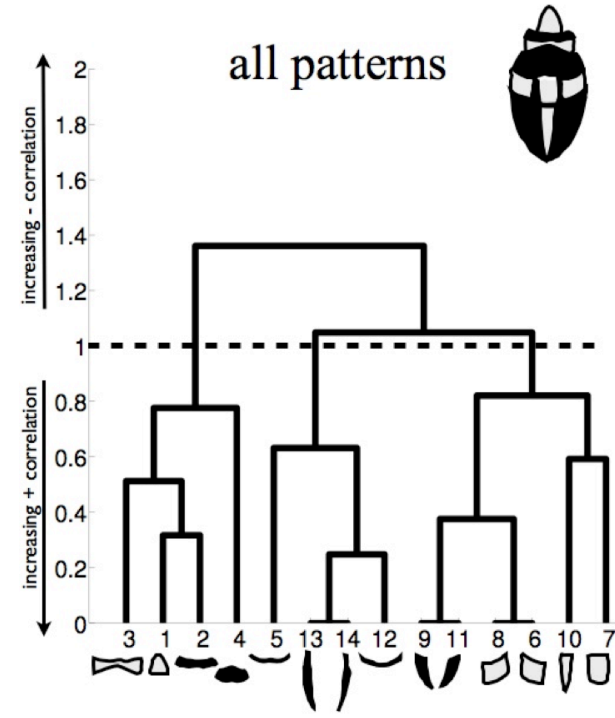

(a)

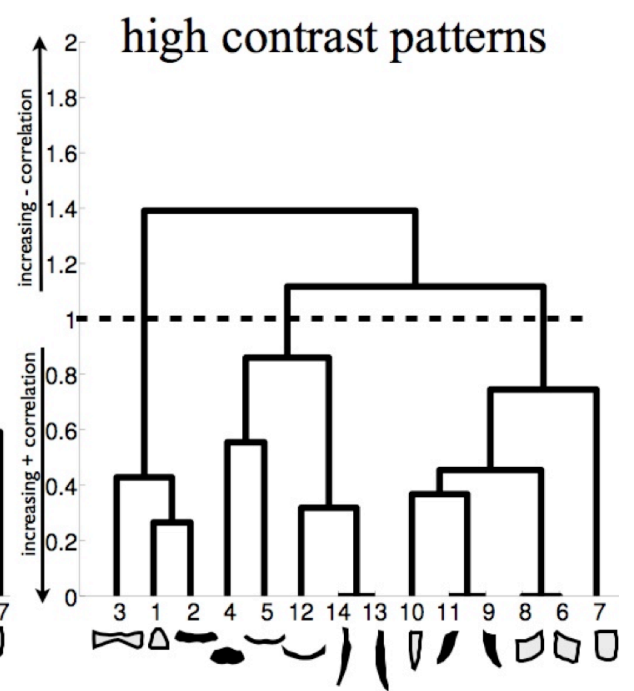

(b)

Figure 5.9 We implemented hierarchical clustering (Duda et al. 2012) for (a) all patterns and (b) high contrast patterns using MATLAB (version 8.2.0.701, R2013b, Mathworks, MA) and used as parameters correlation distance and Ward linkage. Left-to-right placement of components is arbitrary. The vertical height of each leaf indicates the level of correlation between the components on that leaf. The horizontal dashed line indicates the point of no correlation, below which correlations are positive, and above, negative.Components $9 \& 11$, $6 \& 8$ and $13 \& 14$ have perfect correlation since we enforced bilateral symmetry.

\section{Mottleness}

Up to this point, we investigated the functional hierarchy between components only considering their mean intensities. However cuttlefish camouflage patterns are often mottled; that is, they contain small- and large-scale light and dark spots. These spots -are expanded chromatophores, or physiological units of chromatophore patterns (Packard 1972) (Chiao et al. 2010). Chiao and colleagues explain the physiological breakdown of light and dark spots as follows:

"...The large scale component is mainly composed of four sets of Paired Mantle Spots, that surround the white square, whereas the small-scale mottle component is represented by dark splotches of chromatophore aggregations, interspersed with light patches throughout the mantle and arms (resulting from the retraction of chromatopores). By varying the expression strength and frequency of these large and small-scale mottle components, cuttlefish are able to produce a variety of mottle patterns. The size is limited: they only have two sizes, small and large."

We used the images in our database to identify these small and large size mottle spots. After carefully registering the images and warping them onto a template, we manually marked pixels that were consistently dark. Even though warping is potentially an error-prone operation, it was suitable in this case because we only used the warped images to obtain the map in Figure 5.10a, visually verifying the result against the original photographs, and did not use the warped images as inputs into further processing. We were able to obtain the map in Figure 5.10a because the dark spots were approximately in the same locations in the bodies of all five animals used in our experiments. Through 


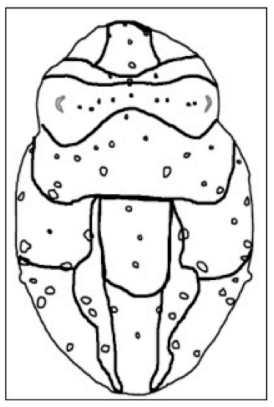

(a)

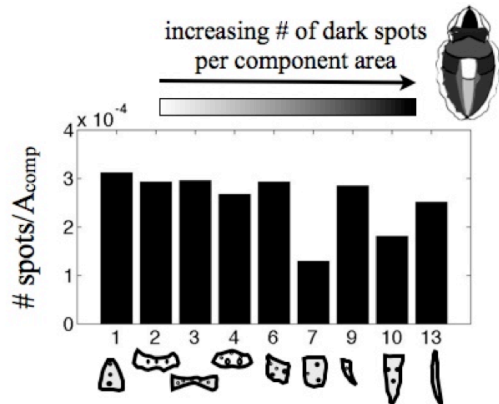

(b)

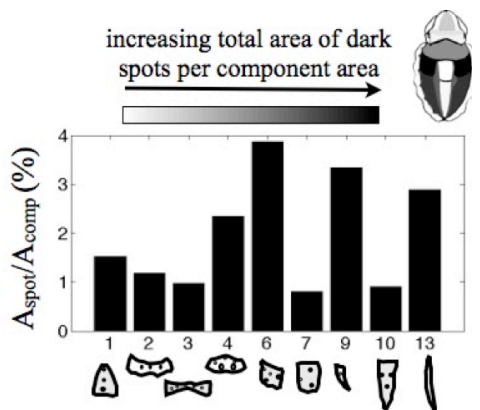

(c)

Figure 5.10 (a) The map of dark mottle spots, or physiological chromatophore units that control the mottleness of patterns, derived by overlaying all images in our database and marking spots that were consistently dark. C5 \& C12 were omitted from this analysis. (b) The ratio of the number of dark spots (\# spots) per component area $\left(A_{\text {comp }}\right)$ is approximately equal for all components, except $\mathrm{C} 7 \& \mathrm{C} 10$. (c) The ratio of the total spot area within a component $\left(A_{\text {spot }}\right)$ to that component's area $\left(A_{\text {comp }}\right)$ shows some variation. Specifically, $C 7 \& C 10$ have the smallest ratio of spot area per component area, followed by the components on the head (C1-3). Components adjacent to $\mathrm{C} 7 \& \mathrm{C} 10$ have the highest ratio $(\mathrm{C} 4,6,9,13)$.

visual inspection this appears to be true for animals from completely different colonies; e.g., see Fig. 2 in Chiao et al. (2010), or Fig. 2 in Allen et al (2010).

There are a few things to note about Figure 5.10. The ratio of the number of dark spots to component area is roughly equal for all components other than $\mathrm{C} 7 \& \mathrm{C} 10$, which also have the smallest ratio of total spot area to component area (i.e., dark spot density). These are light components, which are weakly positively correlated (Figure 5.9a), located on the axis of bilateral symmetry. Their limited mottleness capability might indicate that one of their functions might be to provide a low frequency visual barrier between the right and left halves of a pattern, disrupting the body shape, or may simply be facilitating better background matching against substrates with linear (or, elongated) elements. Components surrounding $\mathrm{C} 7 \& \mathrm{C} 10$, namely $\mathrm{C} 4, \mathrm{C} 6 \& \mathrm{C} 8, \mathrm{C} 9 \& \mathrm{C} 11, \mathrm{C} 13 \& \mathrm{C} 14$, on the other hand, exhibit the highest ratio of dark spot area-to-component area, which suggests that at least for some patterns, the visual effect of separating $\mathrm{C} 7 \& \mathrm{C} 10$ from the rest of the body might be enhanced. Looking at Figure 5.10, it is possible to imagine a pattern with a "ring" of highly mottled components surrounding white and non-mottled components C7 \& C10. But is it possible for cuttlefish to actually show a pattern in which components have different levels of mottleness? In other words, is the mottleness of a given component correlated with, or independent of mottleness of other components? To answer this question, we built a mottleness detector (Figure 5.11) and quantified expression of the mottle spots for each image in our database, at the component level. Our detector is similar to the granularity analysis method of Barbosa et al. (2008b) and Chiao et al. (2010) but differs in that our processing takes place in the spatial domain, and retains the exact locations of the found mottle spots. Chiao and colleagues summed the energy of patterns in 4-6 frequency bands, representing different scales of mottleness with a scalar at each scale. Their work entirely focused only on mottle patterns, and therefore they used background substrates that only evoked mottle patterns; which formed a subset of the patterns used in our study as our goal was to generate as many camouflage patterns as possible. We search for mottle spots at four different spatial 


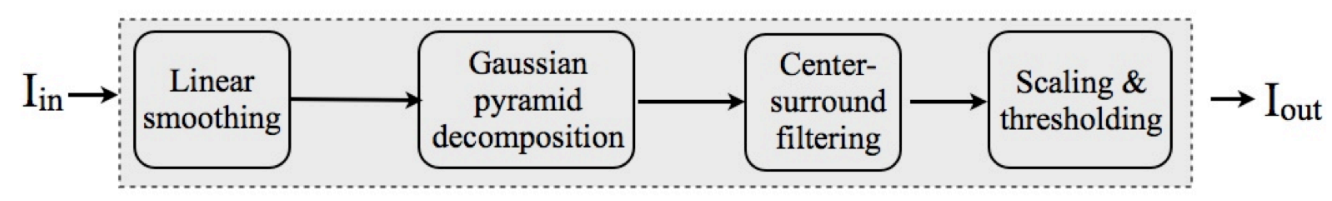

(a)
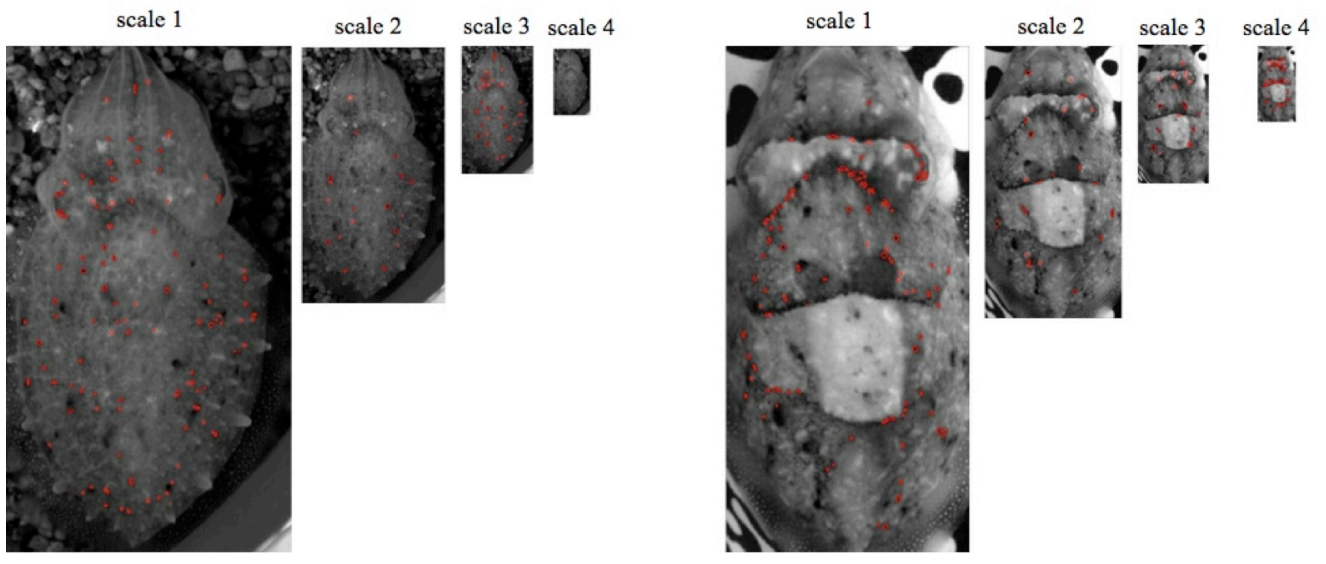

(b)

Figure 5.11 (a) Block diagram for detection of mottle features. Images are first smoothed using a Gaussian filter with $\sigma=5$ pixels (average size of a cuttlefish image is $900 \times 400$ pixels) and then decomposed into a four-layer Gaussian pyramid. At each scale, they are processed by a centersurround filter composed of two concentric dises (inner disc diameter varied from 3 to 9 pixels, outer disc kept at 11 pixels). Filtered results are averaged over all inner diameter sizes, yielding a single filtered image at each pyramid level. [Is there a non-linearity before the second filtering? If not you are effectively running a big, weird, linear filter.] The number of Gaussian pyramid levels is chosen such that the inner disk at the coarsest scale is slightly smaller than the dimensions of the cuttlefish's White Square; ensuring the detection of features smaller than the White Square. As the final step, filtered images at each scale are normalized to fall within the range $[0,1]$ (for display purposes only), and thresholded to eliminate noise components. (b) Examples of some light and dark spots found at different scales.

scales; the finest scale (Figure 5.11b, scale 1) was selected based on the size smallest dark spot we have observed, and the coarsest scale (Figure 5.11b, scale 4) was sized to be just smaller than the animal's White Square (C7). This ensures detection of only light and dark features smaller than $\mathrm{C} 7$ and prevents the expressed White Square from being identified as a large, light mottle spot.

Using the images in our database, we first look for evidence of the two sizes of mottle spots hypothesized by (Chiao et al. 2010). Our mottleness detector used four spatial scales to search for spots rather than assuming that the spots occurred at particular scales. We standardized the area of each dark spot relative to the actual size of the cuttlefish image, and tested whether the spots come from the same distributions. Through visual inspection, we determined that the features identified at scale1 were the same order of magnitude of image noise and excluded this scale from our analysis. We found that the area of spots at scales $2 \& 3$ were log-normal distributed and a two-sample KolmogorovSmirnov test confirmed they were from the same distribution $(\mathrm{p}<0.05)$. This distribution, in turn, was different than that of scale4 (Wilcoxon rank sum, $\mathrm{p}<0.05$ ); indicating that 
there were two scales of spots in our database images. The mean value of small and large spot areas differed roughly by an order of magnitude $\left(\mu_{2} \approx \mu_{3}=-5.01, \mu_{4}=-3.25\right)$.

Next, we used

hierarchical clustering to investigate whether mottle spot expression was correlated across components. We used scale 2 to represent the small-scale mottle spots and scale 4 for large scale. We found that certain components consistently clustered together at small and/or large scales, but the correlations within them were weak $(0.2$ $\leq \rho \leq 0.55)$. Visualizations of these correlations are shown in Figure 5.12. Components around the White Square (C7), namely 6\&8, 9\&11 and $13 \& 14$ clustered together for small and large scale mottle spot expression, while $\mathrm{C} 4, \mathrm{C} 7$ \& C10 formed one cluster for expressing small scale spots. Components on the head (13) expressed large-scale mottle spots together, while their small-scale expression was clustered with the largescale expression of $\mathrm{C} 4$, C7\&C10. The relationship between $\mathrm{C} 7 \& \mathrm{C} 10$ in the expression of mottleness parallels that of intensity.

Finally, we found that

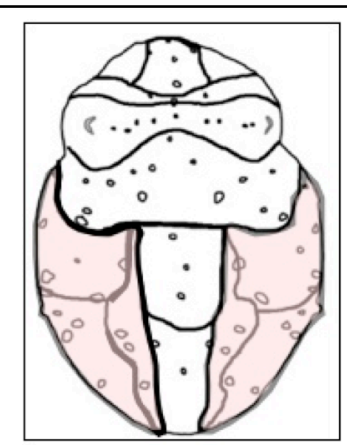

Components that express mottleness together at small and large scales

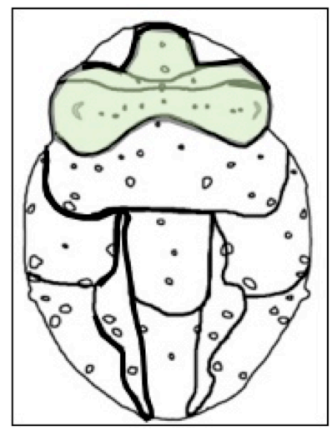

Components that express mottleness together at large scale

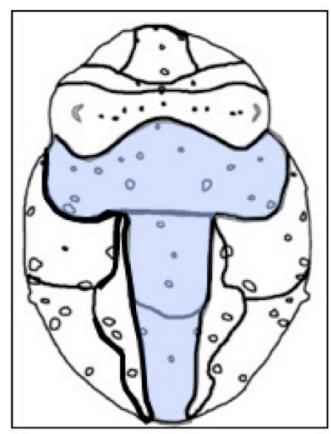

Components that express mottleness together at small scale

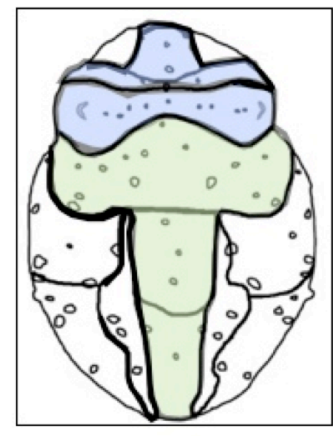

Mixing of small and large scale component mottleness

Figure 5.12 Visualization of clusters of small and large-scale mottle spot correlations across components, based on hierarchical clustering (with parameters correlation distance and Ward linkage) of component dark spot densities. Small and/or largescale spots were expressed in a weakly positively correlated way $(0.2 \leq \boldsymbol{\rho} \leq 0.55)$ in the color coded-clusters. In one case (lower right), the small-scale spots of the head components were correlated with the large-scale spots located on the components on the axis of symmetry $(\mathrm{C} 4,7,10)$.

the difference between the mean areas of small dark and light spots is on the order of $10^{-6}$, supporting the qualitative observations of Chiao et al 2010 that at that scale, the areas of light and dark spots are roughly equal (see Fig. 2 in (Chiao et al. 2010). 


\subsection{Do the space of camouflage patterns form a continuum, or are there a discrete number of clusters? If so, how many?}

Previous attempts to answer these questions have often used subjective judgments by human observers. The human visual system is a powerful pattern analysis machine, however a fundamental disadvantage of determining pattern classes based on human visual texture perception is that inadvertently those properties that are more salient for the human visual system receive more weight, regardless of how they may be perceived by non-human visual systems. But more importantly, our words to describe textures (i.e. lexical texture space) are limited in part because there are more features in textures than those that are important for human visual texture perception (Ravishankar Rao and Lohse 1996). For example, two textures with similar statistics can be found such that it might be very hard for humans to tell them apart even if the patterns in the textures are quite different (Julesz 1962). This discrepancy between visual and semantic spaces arises from the existence of low-level features that computers can extract, but which are not easily perceivable by humans. Thus, limits on human texture perception and texture naming may bias our assessment of the number and type camouflage patterns, if not also supported by objective methods of analysis. Indeed, in their book describing cuttlefish behavior, Hanlon \& Messenger noted that it was difficult to name the body patterns because "they are dynamic and subject to immediate change and countless gradations" (Hanlon and Messenger 1996).

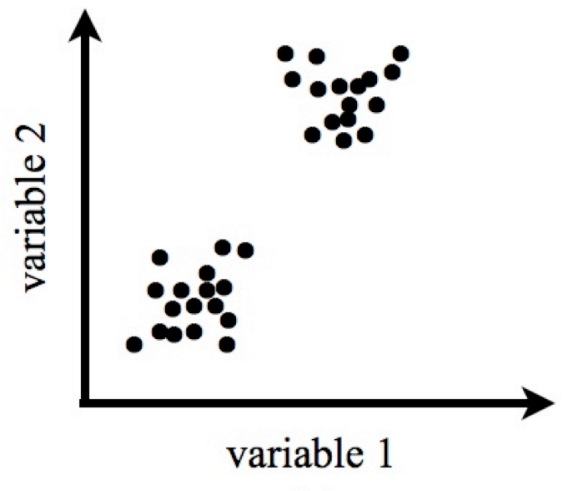

(a)

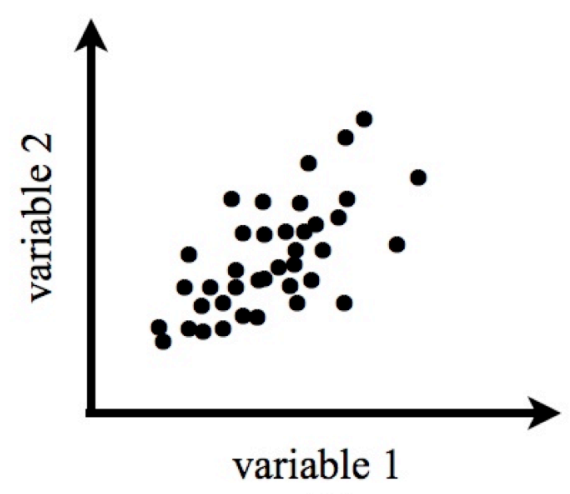

(b)

Figure 5.13 A hypothetical example with bivariate data showing well-separated, discrete clusters in (a) and a continuum in (b).

To quantitatively represent camouflage patterns, we derived an 18-dimensional feature matrix encoding intensity and texture of each pattern. Four of these dimensions carry information about the texture properties (i.e., mottleness) of the overall body pattern, and the remaining 14 are the zero mean intensities of each component. Due to enforced bilateral symmetry, three of these intensities are redundant (components 6,9 and 13 are symmetric with 8,11 and 14 , respectively), which reduces the dimensionality of the feature matrix to 15 . Fifteen dimensions sufficiently represent the properties of each pattern, but pose a visualization challenge. In the simple case of two or three dimensions, we can get intuition about the structure of the data (i.e. whether there are distinct clusters or not) simply by visual inspection (Figure 5.13), but this is impractical when data have 
more than three dimensions. Dimensionality reduction techniques (e.g., principal components or linear discriminant analysis) can be used to project data onto fewer dimensions- usually those retaining the highest variance- making visualization easier and computing faster. However, the retained dimensions may not be optimal for discriminating between clusters and may obscure discrete clusters present in the data. To avoid such manipulation, we investigated the structure of our data directly in 15dimensional space using hierarchical clustering. In general, hierarchical clustering algorithms have complexity $\mathcal{O}\left(n^{3}\right)$ and are computationally prohibitive for highdimensional datasets. However, for a small number of samples as in our case (360) and much smaller number of dimensions (15), this cost is easily manageable $(0.14$ seconds on a $2.3 \mathrm{GHz}$ Intel Core i7 processor).

Figure 5.14a shows that depending on how the dendrogram is partitioned (horizontally); there might be 2-14 well-separated clusters. But closer inspection of patterns shows that none of these clusters are necessarily meaningful because there is great variation within each. We color-coded the branches of the dendrogram to match the ordered thumbnails in Figure 5.14b. Visual inspection shows that within a color-coded clade, patterns have high similarity, but these patterns differ from those in other branches in subtle or significant ways. However, it is clear that if we had chosen a lower threshold to partition the dendrogram horizontally we would have obtained fewer samples in each cluster, but more agreement within clusters.

What, then, is the optimal number of clusters? In the field of pattern classification, it is standard to vary the number of classes the data can be partitioned into, and then assess the stability of each solution. Here, we tested all possible numbers of clusters (ranging between 1 and 360), and looked for a stable solution using the Davies-Bouldin (DB) index (Davies and Bouldin 1979). A stable clustering solution has low within-cluster distances, and high between-cluster distances, yielding a low DB value. Figure 5.14c\&d shows that there is no optimal number of clusters for our data because the DB value keeps falling until every image is in a cluster of its own; i.e. $k=360$.

At this point it is important to note that computational methods like hierarchical clustering will produce clusters for any set of input data. Thus, the interpretation of their outputs is best done based in the context of the research questions asked. For example, are the low-level differences that separate cluster $\mathrm{i}$ from cluster $\mathrm{j}$ perceivable to a particular visual system? If not, they might safely be considered to be a single cluster. For example, a particular predator might be highly sensitive to low spatial frequency contrast, but not as sensitive to contrast in high spatial frequencies. This sensitivity on the part of the observer will affect the distance between patterns and between clusters. The analysis above essentially applies for a visual system as sensitive as our image processing algorithms. Rather than providing a final answer, our methodology and results provide a first-level basis upon which researchers can build. 


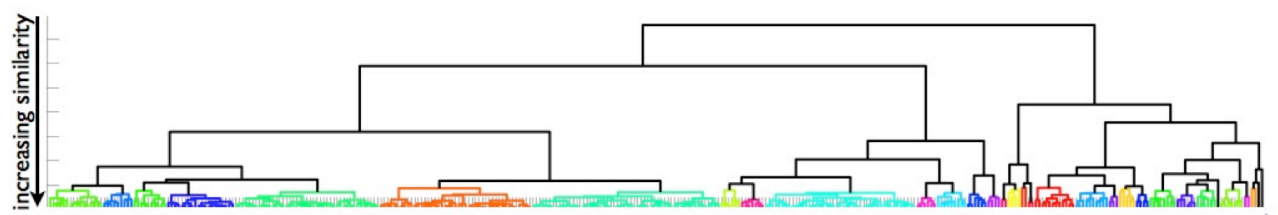

(a)

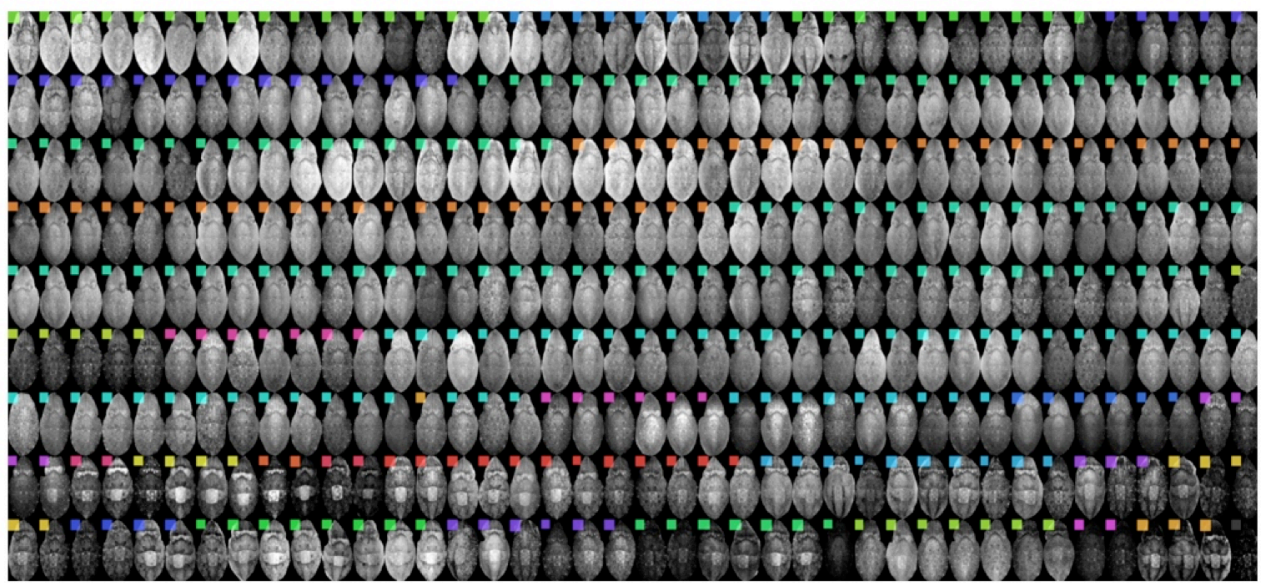

(b)

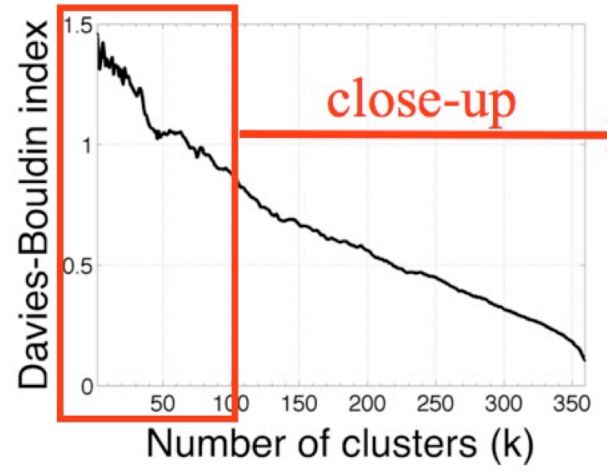

(c)

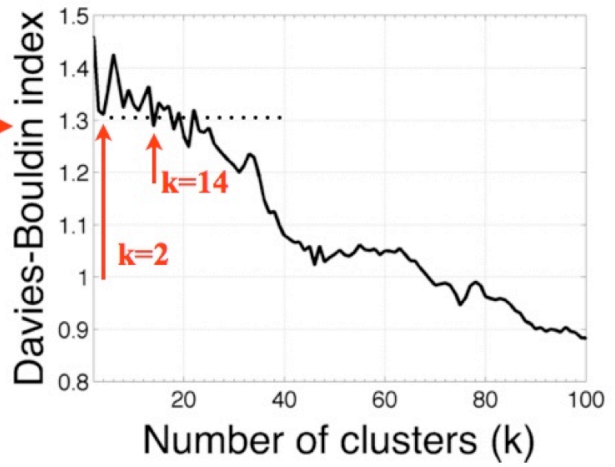

(d)

Figure 5.14 (a) Clustering of the 360 images in our database using intensity and mottleness features. We found the optimal distance (Euclidean distance) and linkage (Ward) parameters using the cophenetic correlation coefficient, which is a validation measure to gauge how faithfully the chosen distance and linkage combinations used for dendrograms represents pairwise distances between the original data points (da Fona Costa and Cesar 2012). (b) Each thumbnail is ordered from left to right, top to bottom, and color-coded to match its location in the dendrogram. Note that mean image intensity was removed from the data used for clustering, while the thumbnails do show variations in absolute mean. This figure can be seen in higher resolution in the supplementary materials. (c) A stable clustering solution should have a low Davies-Bouldin (DB) index. Selecting 2 or 14 clusters has the same DB value, and there is no global minimum. The lowest index is obtained when every image is in a cluster of its own $(k=360)$, indicating that there is no optimal number of clusters. 


\subsection{Do cuttlefish express all possible patterns their components are capable of forming, or do they only show a subset? If so, which ones?}

Body components of cuttlefish are the building blocks of body patterns. This modular structure allows us to enumerate all possible permutations of components to explore the space of all (theoretically possible) body patterns. How many such patterns exist? The answer depends on how body patterns are defined. In the Cuttlefish $72 \times 5$ database image intensities are encoded as 8-bit values; that is, each component is represented using the 256 intensity values between 0 and 255 . If, for simplicity, we omit mottleness altogether, and assume that each of the 11 components expressed intensity independently, a total of $256^{11}=3.1 \times 10^{26}$ patterns would be possible-- a thousand times more than the ratio of atoms per molecules in one mole! Are those patterns all unique? For our research interests, we define a unique pattern to be one that has a distinct spatial layout of elements. In other words, we consider two patterns to be the same even if they vary in mean intensity, as long as they have the same design (i.e., the same $\boldsymbol{I}_{Z M}$ ). One way to investigate if cuttlefish can make all the patterns their components are capable of making, or just a subset, is to look at the principal components (PC) of $\boldsymbol{I}_{Z M}$. For the images in our database, the first six PCs cumulatively explain $96 \%$ of the variance. This suggests that the observed patterns fall in a fairly low-dimensional manifold within the space of all possible patterns; far lower, even, than we would expect if the 360 patterns in our database were each unique.

If cuttlefish cannot show all or most of the patterns they are (theoretically) capable of making, which ones do they make? When mean intensity variations are taken out and only zero-mean images are considered, the number of theoretically possible patterns is reduced significantly by considering only the set of patterns with components at their extreme values; effectively, with each component "On" or "Off”. In Figure 5.15a, we give an example of this for the 2-dimensional case; we call the resulting four patterns templates. The observed patterns, which do contain intensity variations, are plotted in the space defined by these four templates; we plotted 360 such hypothetical patterns in Figure 5.15b (black dots). Next, we'd like to determine to which templates the patterns in our database are most similar. One way to do so is to fit a statistical distribution to the observed patterns (for the hypothetical example in Figure 5.15b, a one-component bivariate Gaussian), and compute the Mahalanobis distance (MD) from this distribution to each of the four templates. The magnitude of the MD is an indication of how likely a particular template might have come from that distribution; the smaller the MD, the more likely.

We extended this approach to the images in our database, obtaining the "On" and "Off" values of each component using their maximum and minimum intensities from Figure 5.5 (adjusted to zero-mean), respectively. This yielded a total of $2^{11}=2048$ unique pattern templates (Figure 5.16). Based on their MD values of the 2048 templates to the distribution that represents our data, we considered patterns in the lowest 5 percentile "most likely", and those in the top 5 percentile "most unlikely" to be produced (Figure 5.17), which coincidentally yielded 102 patterns in both groups. 


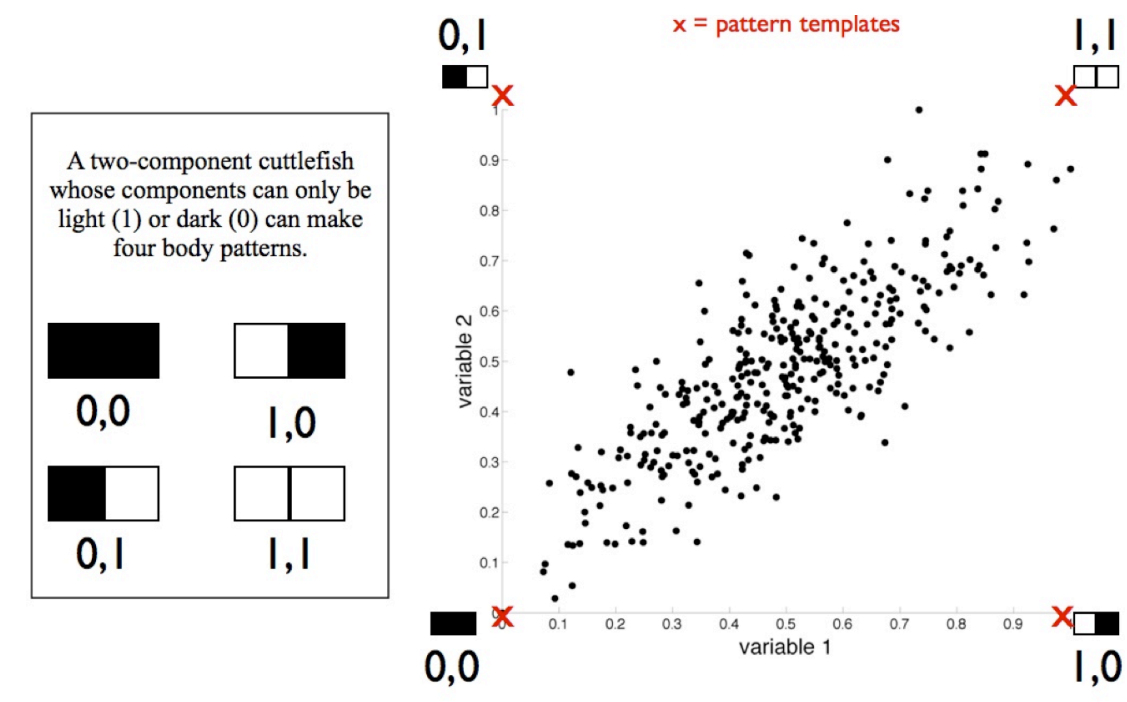

(a)

(b)

Figure 5.15 (a) A simple cuttlefish with two components. If each component can only be "On" (light) or "Off" (dark), there are four possible patterns; we call these templates. (b) The four templates form the boundaries of the space in which hypothetical pattern observations (black dots, $N=360$ ) fall; these patterns show variations in intensity between $[0,1]$. In this example, a bivariate Gaussian distribution is fit to these points. Mahalanobis distances between each template and this distribution are as follows: 0 to $(0,0), 6$ to $(0,1), 3$ to $(0,1)$, and 1 to $(1,1)$. Based on these distances, the all-dark template $(0,0)$ is most likely and $(0,1)$ is most unlikely.

It is useful to study the patterns in Figure 5.17, but grasping the quantitative differences between likely and unlikely patterns and gauging why some patterns were more likely than others this way is difficult. To make this assessment easier, in Figure $5.18 \mathrm{a} \& \mathrm{~b}$ we present visualizations of the PCs of the most likely and unlikely patterns. At a glance, these visualizations show that the first PC of the "most likely" set effectively facilitates high contrast between the White Square and the components on the head. Patterns with such combinations account for the biggest chunk of the variance in our dataset. In complement, the first PC of the "most unlikely" set is the lack of contrast between the White Square and the head components, i.e., their correlated expression. We study this PC in more detail to see if we can gain any insights towards understanding what made the patterns in Figure 5.17 likely or unlikely. In Figure 5.18c, we re-plot the pairwise correlations between $\mathrm{C} 1-3$ and $\mathrm{C} 7$ from Figure 5.7a, and overlay on top all likely and unlikely templates (red x's). From this visualization, it becomes clear that throughout the likely templates, the White Square is expressed almost entirely within its limits observed in our database; specifically, we have observed that $\mathrm{C} 1-3$ can be expressed dark when C7 is light, and C1-3 can be expressed dark when C7 is dark. The likely templates follow this pattern. Throughout the unlikely templates, we notice the opposite: with the exception of outlier points, we did not observe C1-3 to be expressed light when $\mathrm{C} 7$ is light; and we did not observe $\mathrm{C} 1-3$ to be light when $\mathrm{C} 7$ is dark. The "most unlikely" templates capture these relationships. In fact, the relationship between all other components and C7 in the likely and unlikely templates mirror the pattern shown in Figure 5.18c. 
We should note here that a pattern ranked unlikely by our analysis does not mean that it is an impossible pattern. A pattern may have been assigned to the unlikely pile for two reasons: it had a very low likelihood of being produced based on the component relationships in our dataset, or it was not observed in our database at all. Thus, it is possible to refine the space of all possible patterns cuttlefish can make by designing background substrates that might evoke the unlikely patterns, or by considering images from the wild where cuttlefish are subject to complex habitats and in turn may show different patterns.

\subsection{Discussion}

In this work, we quantified cuttlefish camouflage patterns from photographs, ensuring that our data acquisition process and methodology were maximally objective. To do so, we minimized involvement from human observers, used calibrated linear photographs, and avoided image-processing steps that might introduce artifacts. Our goal was to derive an objective feature matrix that represents cuttlefish body patterns, which could then be used to answer more complex questions. We focused on three such questions in particular; the relationship between the level of expression of components, the structure of the camouflage pattern space, and the total number of patterns that might be possible for cuttlefish to produce. We found that $\mathrm{C} 7 \& \mathrm{C} 10$, which are on the axis of bilateral symmetry, are correlated for both intensity and mottleness expression. In addition, these light components have the smallest number are area density of dark mottle spots, indicating that one of their functions might be to be expressed lighter and less mottled than the surrounding components, forming a vertical stripe between the right and left sides, visually disrupting the animal's body outline. This result may be considered to be the first computational evidence towards determining whether cuttlefish utilize two camouflage strategies: background matching and disruptive coloration.

Based our analyses, we propose two updates to the terminology currently used to describe cuttlefish camouflage patterns. First, we found that mottle features can be present on every pattern, suggesting that mottleness is a pattern feature, rather than Mottle being a unique pattern type. Second, the lack of strong correlations between all "light" and "dark" components suggests that there is no single high-contrast pattern, and components can combine in numerous ways to make such patterns. High contrast patterns have historically been termed Disruptive; yet as we have shown in in Figure 5.8b, there exist high-contrast patterns in which components like $\mathrm{C} 7 \& \mathrm{C} 10$ that likely disrupt body outline are not expressed as disruptive elements. Even when they are expressed, the term Disruptive more appropriately describes a subset of high-contrast patterns rather than one unique design.

The body patterns in our data do not clearly group into discrete and wellseparated clusters. The space of patterns observed in our database, based on the features we have derived, appear to form a continuum. In many ways, this result is not surprising because both intensity and mottleness are features that can be expressed at varying levels, in an intertwined fashion, suggesting that many subtly different patterns can be made.

Furthermore, our work only serves as the first step towards understanding whether the continuum-like behavior of cuttlefish patterns implies that cuttlefish have only one mechanism of producing camouflage patterns, and therefore may (or may not) employ only one type of camouflage strategy. Future work involves the perception of patterns 
through observer-specific visual systems, which may reveal that with respect to a particular observer's visual system some differences between patterns may not be discriminable, and patterns may be grouped into fewer classes for that observer.

The number of images in our database (360) was an order of magnitude smaller than the number of theoretically possible patterns we hypothesized cuttlefish could show (2048). Since we used as basis the images in our database, the properties of patterns that were unlikely to be made reflect what might be underrepresented in our database. A future task would be to design substrates that will specifically evoke some of the unlikely patterns and/or use photographs of cuttlefish patterns from the wild, which may show differences than those captured in the lab.

While our work was based entirely on images, it should be noted that the biological and evolutionary constraints on the design of the nervous system and the skin of cuttlefish must be considered. In any biological system that can produce more than a single camouflage pattern, there must be a sensory system to assess the background, a brain to integrate and synthesize the information, and a motor control system that produces the patterns in the skin. These are major constraints that might help explain why relatively few animals have evolved such a capability. A key question that has guided much of the biological investigation of rapid adaptive camouflage in cephalopods is: how do they so rapidly assess and implement their pattern on such diverse backgrounds such as coral reefs (Hanlon 2007)? There must be some parsimonious solution involved because there is too much visual information available to them, and too many variations of patterns that they could produce with so many chromatophores and iridophores (millions of each in a single cuttlefish); controlling such a vast amount of information would take a truly enormous brain, presumably much larger than what they actually have. We hope that our work will inspire more biological and neuro-physiological research to understand the body component relationships and constraints, which could then lend insights to evolution of cuttlefish's camouflage patterns.

\subsection{Conclusion}

We derived intensity and mottleness features from photographs of S. officinalis camouflage patterns and used these to answer three main research questions. We used 72 different background combinations and 5 adult cuttlefish to build the Cuttlefish $72 \times 5$ database that contains 360 calibrated images. Through the analysis of these images, we showed that every body component showed a wide range of (light-to-dark) intensities, despite being labeled as "light" or "dark", except for the White Square, which was dominantly light. We found that the components on the head and body were often expressed in contrasting intensities suggesting that the head and the mantle may be used as two different parts of a pattern employing different strategies. We also found that $\mathrm{C} 10$ had high correlation with $\mathrm{C} 7$ in high-contrast patterns, making those components essential for the creation of contrast.

We custom built a mottleness detector to quantify the expression of light and dark spots present in pattern at the component level. Through the analysis of all the images in our database, we made a map of the chromatophore units that control the expression of dark spots. These spots appear to be in the same locations for all animals. Dark spots identified by our mottleness detector quantify the descriptive statement by Chiao et al 
(2010) that the cuttlefish skin only has two sizes of mottle; small and large. We found that each component has approximately the same number of dark spots per its area, with the exception of C7\&C10, which have less. Similarly, C7\&C10 have the least total dark spot area per component area when compared to other components. This relationship between $\mathrm{C} 7 \& \mathrm{C} 10$ parallels a similar one in intensity, suggesting that perhaps these light components, located on the axis of bilateral symmetry, function as a visual barrier between the right and left halves of a pattern, disrupting the animal's shape, or help blend in better with the elongated features of a background. We also found that the total area of small light and dark spots are roughly equal, confirming the visual observation made by Chiao et al (2010).

Using an 11-dimensional feature vector composed of intensity and mottleness, we found that the 360 patterns in our database did not discrete, well-separated clusters. Finally, based on the inter-component correlations we observed, we conclude that cuttlefish are likely to have physiological limitations that prevent them from producing every theoretically possible body pattern. 

Hef

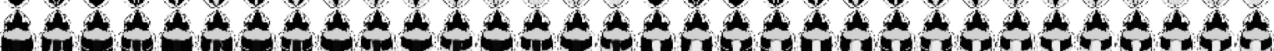

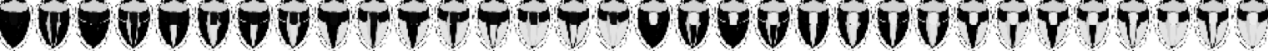

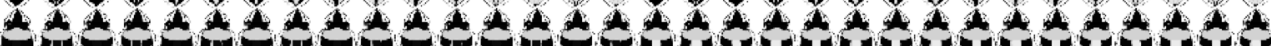

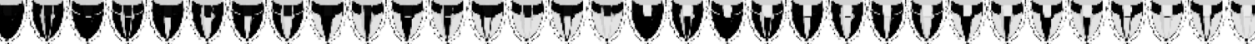

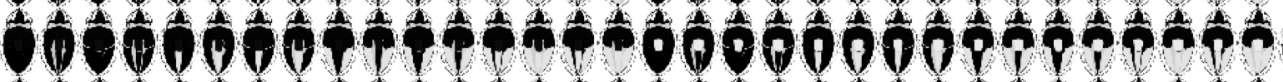

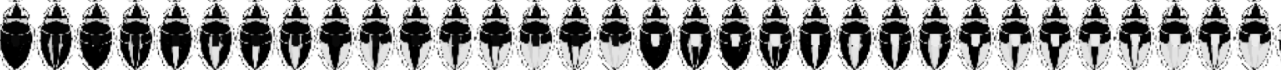

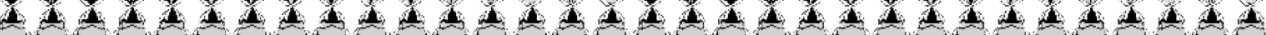

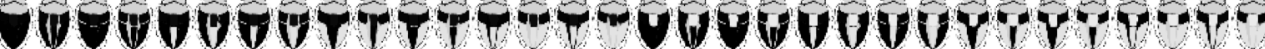

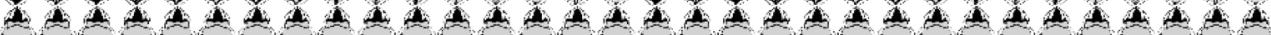
of

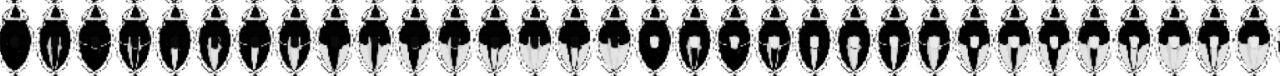

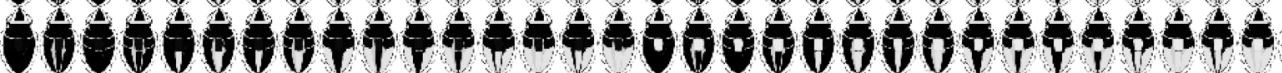

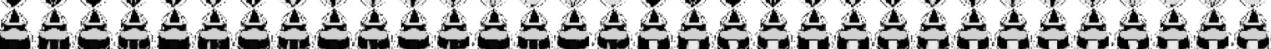
W.

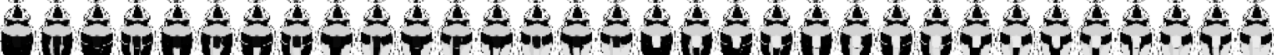

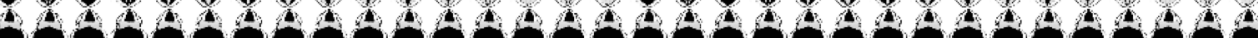
W W.

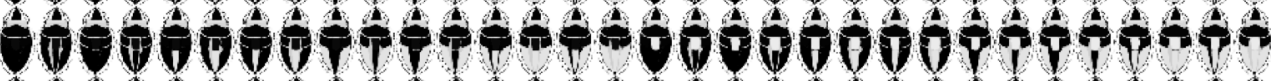

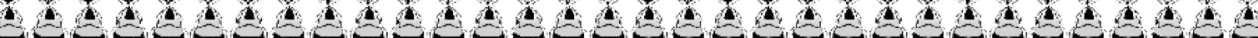

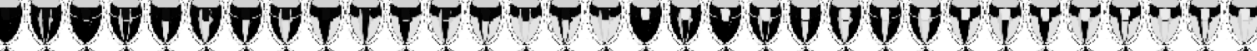

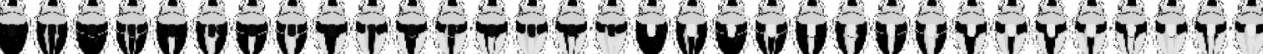

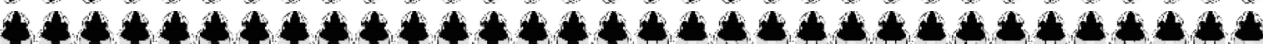

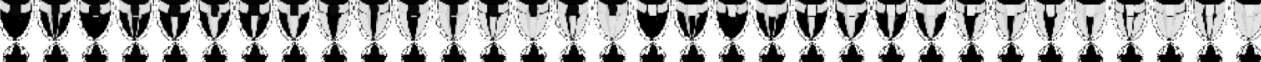

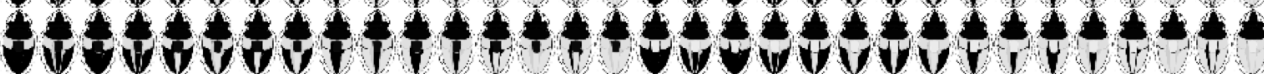

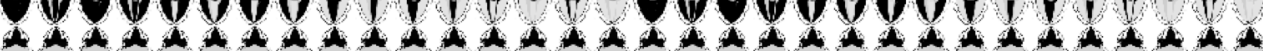

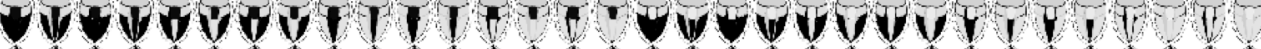

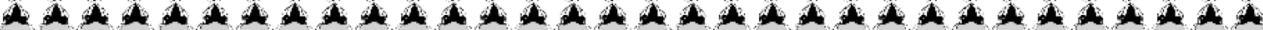

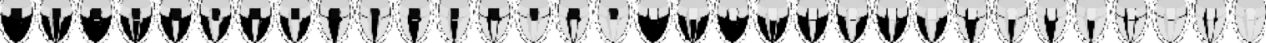

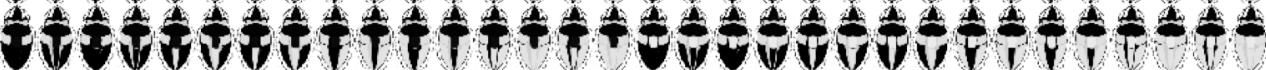

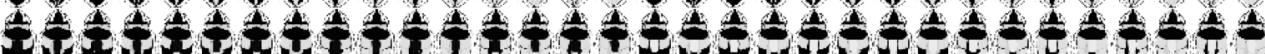

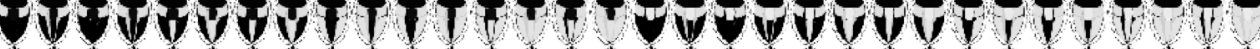

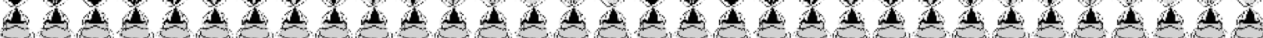

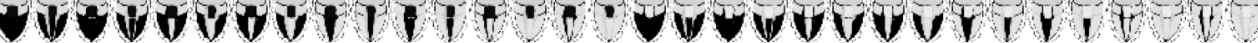

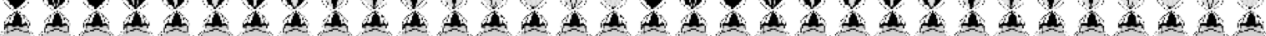

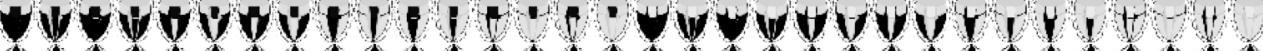

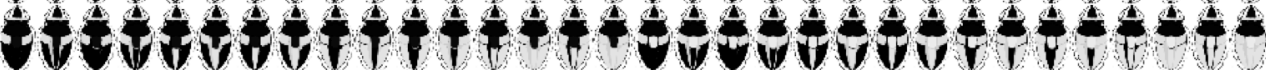

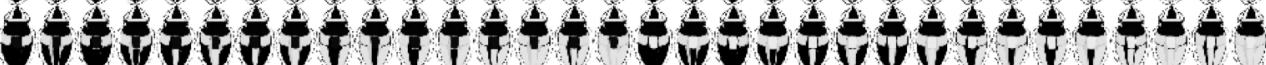
$\angle 4 \leq 4 \leq 4 \leq 4 \leq 4 \leq 4 \leq 4 \leq 4 \leq 4 \leq 4 \leq 4 \leq 4 \leq 4 \leq$

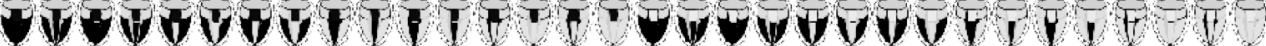
4242424242424242424242424242

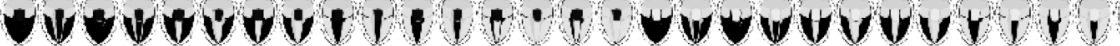

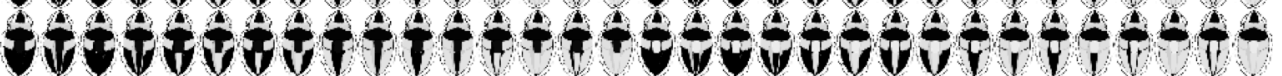

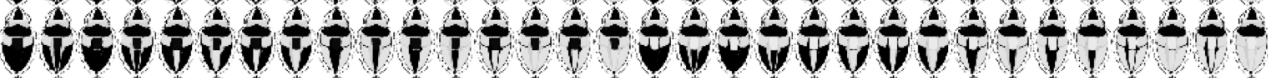

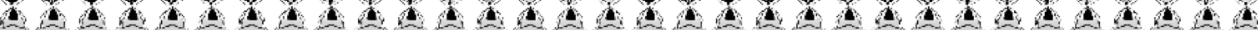

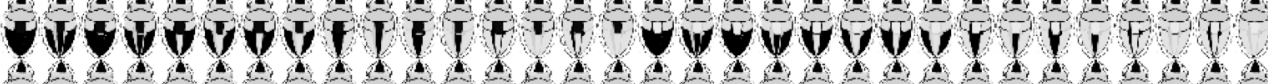
wVG

Figure 5.16 Binary cartoons of 1024 of the 2048 patterns cuttlefish can theoretically make through permutation of component expressions. The remaining 1024 patterns are the opposite polarity versions of those shown here. 

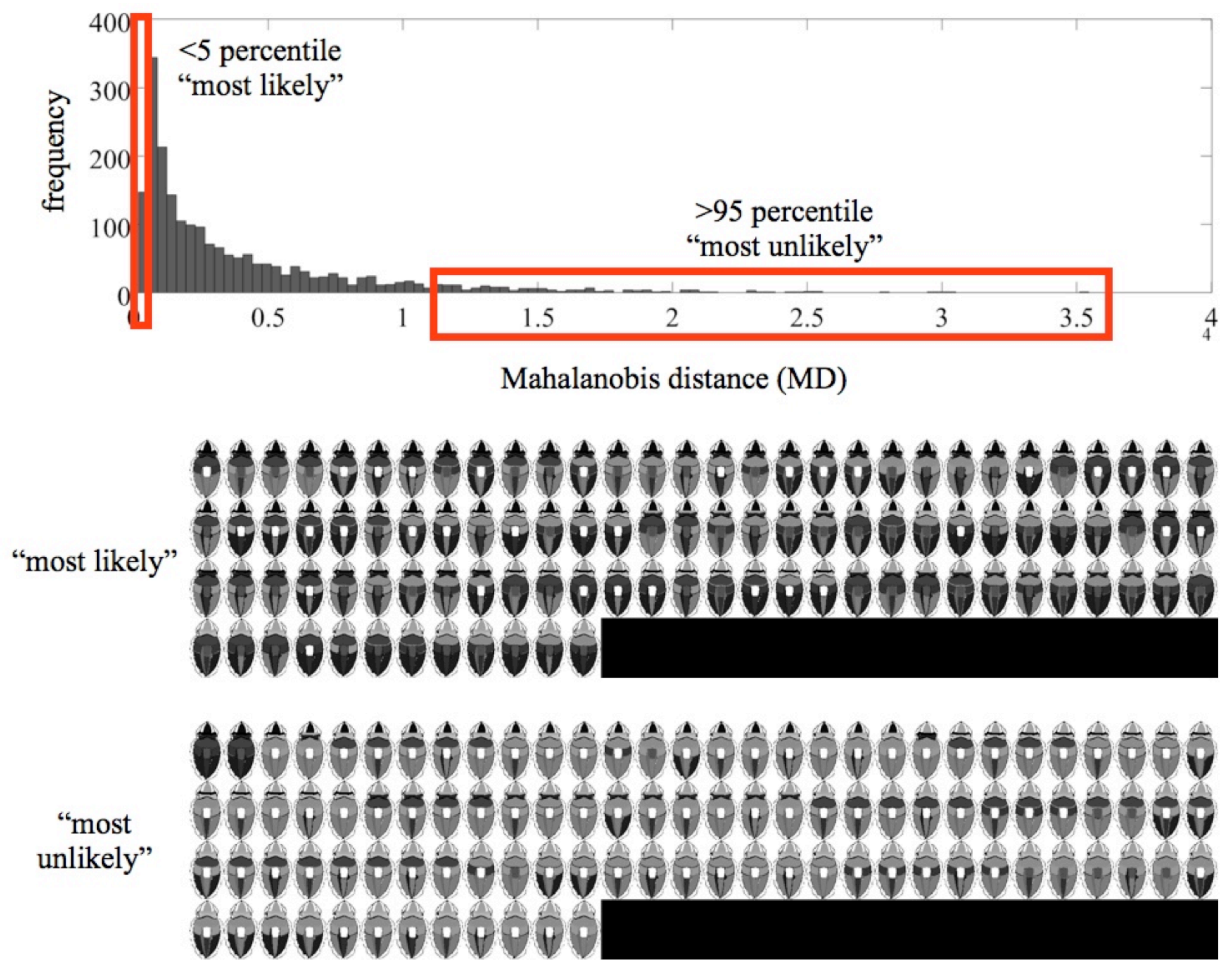

Figure 5.17 The Mahalanobis distance (MD) between the multivariate distribution fit to $I_{Z M}$ and each of the 2048 theoretically possible patterns is a measure of how likely these patterns were observed in our database. We defined those in the $5^{\text {th }}$ percentile, having the smallest MD, as "most likely", and those in the $95^{\text {th }}$ percentile, having the largest MD, as "most unlikely". 


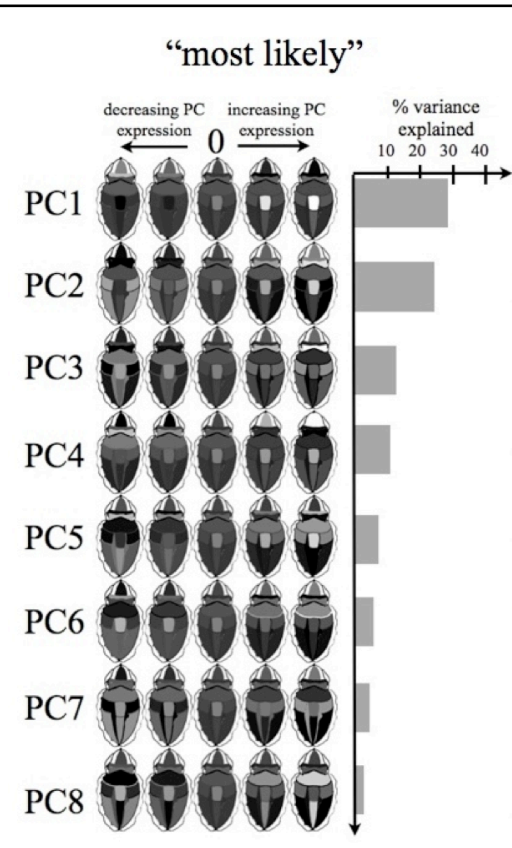

(a)

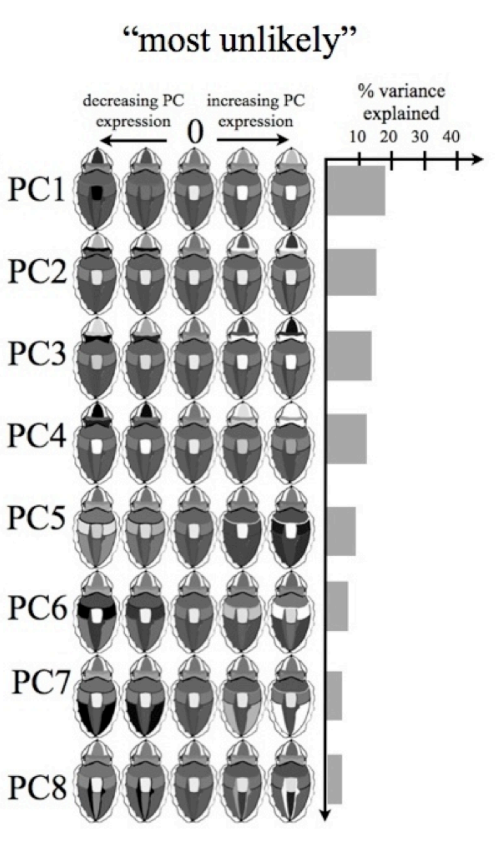

(b)
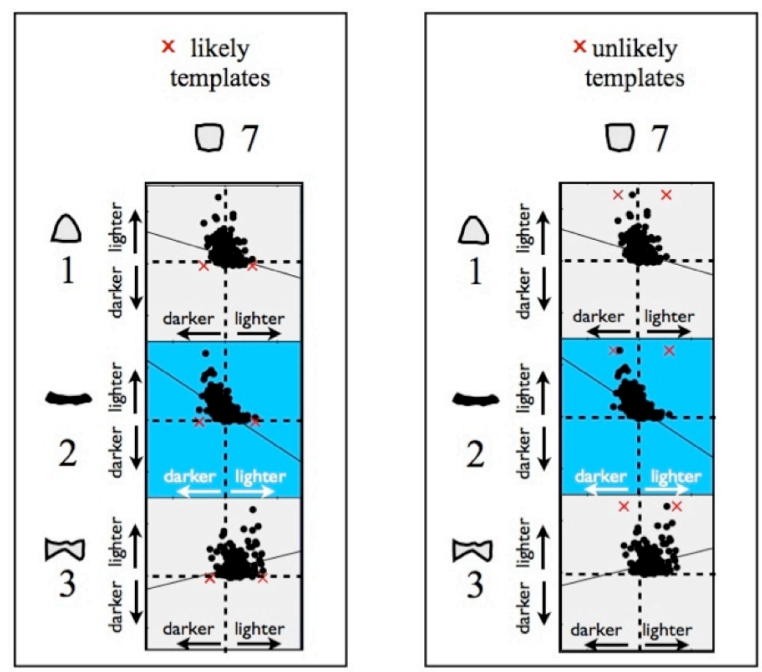

(c)

Figure 5.18 Visualization of the features each principal component (PC) controls in the (a) most likely and (b) unlikely templates; 8 principal components retained $89 \%$ and $72 \%$ of the variance, respectively. Note that these cartoons do not represent actual body patterns, and only show the positive and negative contributions of each principal component relative to the average pattern; e.g., a dark White Square does not imply that the White Square can be expressed that way. The 1 st PC of the "most likely" set facilitates high contrast between the C7 and C1-3, and the opposite in the "most unlikely" set; their correlated expression. We study this PC in detail in (c). The pairwise correlation plots from Fig. 36a are re-plotted here for $\mathrm{C} 1-3$ and $\mathrm{C} 7$ and the red $\mathrm{x}$ 's show where in this these distributions the templates fall. The $\mathrm{C1-3}$ and $\mathrm{C} 7$ relationships in the likely templates parallel those observed in our database well; while those in the unlikely set represent relationships we did not observe in our database; such as C1-3 being expressed light when $\mathrm{C} 7$ is light, and $\mathrm{C} 1-3$ being light when $\mathrm{C} 7$ is dark. 


\section{Chapter 6}

\section{Contributions}

- Established a framework for the use of commercial-off-the-shelf cameras as scientific data acquisition tools including, but not limited to, their use in the field of animal coloration and camouflage. This framework ensures consistent color capture and can be used on land or underwater.

- Established "scene-specific color calibration" to maximize color capture accuracy of commercial-off-the-shelf cameras, applicable in every field of science including, but not limited to, animal coloration and camouflage. This method can be used on land or underwater; its use for underwater scenes can offer up to 20fold decrease in color transformation errors.

- Derived the equations governing the freehand use of optical fibers that determine the optimal measurement distance and angle from the surface of a sample. These equations can serve as guidelines for determining how close a surveyor should expect to get to the surface being measured; e.g., skin of an animal, to record uncontaminated spectra from a feature of known size. Alternatively, for existing measurements, they could quantify the degree of contamination if measurement angle and distance were recorded as a part of the field study.

- Quantified the effect of contaminated spectra on discriminability of colors in the eyes of non-human animals for the assessment of camouflage effectiveness. Showed that the use of contaminated spectra for camouflage assessment could lead to (incorrectly) concluding that two colors with minimal spectral overlap were indistinguishable to a particular visual system.

- Quantified, for the first time, the color match of European cuttlefish in the eyes of its predators using reflectance and irradiance spectra measured in situ. Showed that cuttlefish matched colors in their habitats closely; but the particular habitat studied in the Aegean Sea was not colorful.

- Built reflectance and irradiance spectra databases characterizing underwater habitats in Urla (Turkey), Kona (Hawaii) and Woods Hole, (MA). These databases can be used for future color matching studiesd, as well as bio-diversity and ecological surveys, and color-sensitive underwater imaging applications.

- Built the first database (to be made publicly available) containing calibrated linear photographs of five adult camouflaged cuttlefish, taken against 72 backgrounds; a comprehensive superset that included all backgrounds used for previous studies as well as new ones. This database also contains time-lapse photographs of cuttlefish's settling behavior until a stable camouflage pattern is shown. 
- Designed a "mottleness detector" for cuttlefish body patterns (also applicable to other animal patterns): a spatial filter that identifies dark and light spots at four different scales. This detector offers an advantage over existing ways of quantifying mottleness through capturing the spatial locations of dark and light spots, as well as their size and shape. This enables the study of more advanced questions, such as which elements form physiological units in the skin of the cuttlefish that contribute to the consistent expression of mottleness.

- Derived a high-dimensional feature vector representing cuttlefish camouflage patterns, consisting of both intensity and mottleness features. This feature vector, which was derived using objective and repeatable computational tools, facilitates the study of complex questions regarding cuttlefish camouflage, such as how patterns differ from each other or how many patterns there might be.

- Quantified correlations between chromatic body components of European cuttlefish in terms of their levels of expression of intensity and mottleness. Most recent physiological studies do not address how cuttlefish body components function to generate overall patterns. Our data-driven approach forms a baseline for future physiological studies that might investigate component relationships using state-of-the-art techniques.

- Employed pattern recognition techniques to investigate the structure of the highdimensional space of cuttlefish patterns and found they formed a continuum rather than well-separated discrete clusters.

- Used an image-based approach to perform a preliminary analysis regarding physiological limitations cuttlefish may have when combining chromatic body components to create overall camouflage patterns. Found that there appear to be constraints to the patterns cuttlefish can show, providing a baseline for future electro-physiological studies. 


\section{Chapter 7}

\section{Future Work}

In this thesis, we focused on visual camouflage based on animal patterns, specifically those of cuttlefish (S. officinalis). In biology, there is no widespread consensus on the definitions of camouflage strategies, which partly stems from our lack of knowledge of the perceptual mechanisms of the relevant observers, but also from lack of publicly available data (e.g., RGB or multi-spectral photographs or point-by-point spectral measurements) that is free of instrument bias. The establishment of the Cuttlefish $72 \times 5$ database (Chapter 5) will facilitate deeper analyses of camouflage strategies, as well as biological questions related specifically to European cuttlefish. In particular, using the Cuttlefish $72 \times 5$ database, we will be able to analyze the time-dependent settling behavior of cuttlefish and gain insights into how the final camouflage pattern may be chosen. This behavior will also be integrated with the statistics and spatial layout of the background substrates.

Through quantifying the dark mottle spot distribution across each body component, and investigating their correlated expression, we found evidence that cuttlefish might be physiologically wired to utilize two camouflage strategies- background matching and disruptive coloration. Our feature matrix consisting of intensity and mottleness provides a first-level basis upon which researchers can build. For example, we found that using these features extracted from our dataset, we cannot classify cuttlefish patterns into wellseparated classes. For future work, a predator's visual system can be taken into account. Certain patterns that showed quantifiable differences might become indistinguishable because that predator may not be sensitive to those features. Then, from the point of view of that visual system, it would be possible to classify patterns into discrete groups. We also examined what the theoretical number of patterns cuttlefish can produce might be, and found that cuttlefish likely show a much smaller subset of what their components are capable of showing. Given the theoretical space of all possible patterns, and those patterns we actually observed in our database, we found that some patterns more likely to be produced than others. An important task for future work would be to design background substrates that might evoke some of the patterns that were classified as unlikely.

As a part of our work investigating biological questions related to cuttlefish camouflage, we have made contributions towards the acquisition of unbiased data using conventional optical instruments. In Chapter 2, we presented a framework that enables consistent and accurate color capture using commercial-off-the-shelf digital cameras. These cameras have applications in all fields of science and following this framework to obtain properly calibrated photographs can open up new uses for them. For example, some applications of the scene-specific color calibration method described here can be in producing color-corrected photomosaics (underwater, or land); in mineralogy where minerals in soil could be classified from photographs; in medical imaging when foot sore, or ulcers could be imaged by patients at home and communicated to medical professionals digitally, or for ecological monitoring projects similar to the coral health example we have used in Chapter 2. 
In Chapter 3, we derived an equation that determines the degree of spectral contamination when the spectrometer fiber is held too far away from the object being measured, or at too steep of an angle. This equation depends only on the numerical aperture and diameter of the optical fiber, which are always known, therefore it can be used as a guideline to come up with a measurement distance and angle estimate before carrying out fieldwork (e.g., relative to the size of spots that will be measured on an animal) or any kind of study that requires free-hand use of an optical fiber. Since this equation only depends on geometrical optics, it is applicable for spectroscopic measurements in every field.

In Chapter 4, we presented the first study that used unbiased spectrometer measurements taken in situ in the Aegean Sea, comparing S. officinalis pattern colors to those of its natural habitat (Akkaynak et al. 2013). We found that cuttlefish showed a good match to colors in their habitats but the range of colors in the particular Aegean habitat we studied was indeed limited. For future work, it would be an interesting experiment to transport cuttlefish from one habitat, e.g., the Aegean, to a more colorful habitat, such as a coral reef, to examine whether their ability to match color is affected when the gamut of their habitat colors changes noticeably. In addition, based on spectroscopic measurements made from cuttlefish skin, including microscopic measurements of chromatophores previously done by Mäthger et al. (2008), the gamut of all colors cuttlefish can (theoretically) produce can be derived, establishing a basis of comparison with the chromatic properties of natural habitats.

Quantification and interpretation of animal camouflage patterns have significance and applications beyond behavioral and evolutionary biology. For example, understanding what features of a background an animal's pattern matches, successfully deceiving the observer's visual system, would give us the ability to replicate it with an option to tune the degree of camouflage, making the target better or worse camouflaged. For example, such control could be used to magnify or reduce how well a feature blends in with a digital artwork, like a company logo on a poster. Furthermore, understanding the dimensions that control camouflage would give us insights to reverse engineer patterns and use that knowledge expose hidden target(s) in images. In addition to defense, camouflage-breaking techniques have natural applications in medical imaging, where cancerous tissue is often difficult to segment from healthy tissue, e.g., in mammograms, endoscopy or colonoscopy images. 


\section{Appendix}

In 3-space, $\frac{x^{2}+y^{2}}{(R / H)^{2}}=z^{2}$ defines a right cone with its apex on the origin $O(0,0,0)$, whose base is a circle with radius $R$ in the XY plane, located at a distance $H$ away from the apex along the z-direction. A plane that intersects this cone at height $H_{s}$, at an angle $\alpha$ is defined by $z=\tan \alpha \cdot y+H_{s}$. These two equations are solved together to find their intersection:

$$
\frac{x^{2}+y^{2}}{(R / H)^{2}}=\left(\tan \alpha \cdot y+H_{s}\right)^{2}
$$

Expanding the terms on the right hand side and re-arranging gives

$$
\frac{x^{2}}{(R / H)^{2}}+y^{2}\left(\frac{1}{(R / H)^{2}}-\tan ^{2} \alpha\right)-2 \tan \alpha H_{s} y=H_{s}^{2}
$$

Defining $t=R / H$ and $M=\frac{1}{t^{2}}-\tan ^{2} \alpha$ yields:

$$
\frac{x^{2}}{M t^{2}}+y^{2}-\frac{2 \tan \alpha H_{s}}{M} y=\frac{H_{s}^{2}}{M}
$$

Completing the square, the last equation reduces to

$$
\frac{x^{2}}{M t^{2} C}+\frac{\left(y-\frac{H_{S} \tan \alpha}{M}\right)^{2}}{C}=1
$$

where $C=\frac{H_{S}^{2}}{M}\left(1+\frac{\tan ^{2} \alpha}{M}\right)$. From this, we obtain the center of the ellipse as $\left(x_{c}, y_{c}\right)=$ $\left(0, \frac{H_{S} \tan \alpha}{1 / t^{2}-(\tan \alpha)^{2}}\right)$, with semi-major axis $a=\sqrt{C\left(1-t^{2}(\tan \alpha)^{2}\right)}$ and semi-minor axis $b=\sqrt{C}$. A quick check shows that when $\alpha=0\left(x_{c}, y_{c}\right)=(0,0)$ and $a=b=\sqrt{C}$, making a circle parallel to the base of the cone. . 


\section{Bibliography}

Åhlén J (2005) Colour correction of underwater images using spectral data. Thesis, Uppsala University.

Akkaynak D (2014) Use of spectroscopy for assessment of color discrimination in animal vision. J Opt Soc Am A 31 (4):A27-A33. doi:10.1364/josaa.31.000a27

Akkaynak D, Allen J, Mäthger L, Chiao C-C, Hanlon R (2013) Quantification of cuttlefish (Sepia officinalis) camouflage: a study of color and luminance using in situ spectrometry. J Comp Physiol A 199 (3):211-225. doi:10.1007/s00359-0120785-3

Akkaynak D, Chan E, Allen JJ, Hanlon RT (2011) Using spectrometry and photography to study color underwater. Proceedings of IEEE OCEANS, Kona, HI USA, pp 1-8

Akkaynak D, Treibitz T, Xiao B, G,rkan UA, Allen JJ, Demirci U, Hanlon RT (2014) Use of commercial off-the-shelf digital cameras for scientific data acquisition and scene-specific color calibration. J Opt Soc Am A 31 (2):312-321. doi:10.1364/josaa.31.000312

Allen JJ, Mäthger LM, Buresch KC, Fetchko T, Gardner M, Hanlon RT (2010a) Night vision by cuttlefish enables changeable camouflage. The Journal of Experimental Biology 213 (23):3953-3960

Allen JJ, Mäthger LM, Barbosa A, Buresch KC, Sogin E, Schwartz J, Chubb C, Hanlon RT (2010b) Cuttlefish dynamic camouflage: responses to substrate choice and integration of multiple visual cues. Proceedings of the Royal Society B 277:10311039. doi:FirstCite doi:10.1098/rspb.2009.1694

Allen JJ, Mäthger LM, Barbosa A, Hanlon RT (2009) Cuttlefish use visual cues to control three-dimensional skin papillae for camouflage. J Comp Physiol A 195 (6):547-555

Allen WL, Higham JP (2013) Analyzing Visual Signals as Visual Scenes. American J of Primatology 75(7):664-682

Alsam A, Finlayson G (2008) Integer programming for optimal reduction of calibration targets. Color Research \& Application 33 (3):212-220

Anderson J, Baddeley R, Osorio D, Shashar N, Tyler C, Ramachandran V, Crook A, Hanlon R (2003) Modular organization of adaptive colouration in flounder and cuttlefish revealed by independent component analysis. Network: Computation in Neural Systems 14 (2):321-333

Aristotle (1910) Historia Animalium (trans: Thompson DAW). Clarendon Press, Oxford

Arnold SE, Faruq S, Savolainen V, McOwan PW, Chittka L (2010) FReD: the floral reflectance database,A web portal for analyses of flower colour. PloS one 5 (12):e14287

Baldwin J, Johnsen Sn (2012) The male blue crab, Callinectes sapidus, uses both chromatic and achromatic cues during mate choice. The Journal of Experimental Biology 215 (7):1184-1191

Barbosa A, Allen JJ, Mäthger LM, Hanlon RT (2012) Cuttlefish use visual cues to determine arm postures for camouflage. Proceedings of the Royal Society B: Biological Sciences 279 (1726):84-90

Barbosa A, Litman L, Hanlon RT (2008a) Changeable cuttlefish camouflage is influenced by horizontal and vertical aspects of the visual background. J Comp Physiol A 194:405-413 
Barbosa A, Mäthger LM, Buresch KC, Kelly J, Chubb C, Chiao C-C, Hanlon RT (2008b) Cuttlefish camouflage: The effects of substrate contrast and size in evoking uniform, mottle or disruptive body patterns. Vision Research 48:1242-1253

Barbosa A, Mäthger LM, Chubb C, Florio C, Chiao CC, Hanlon RT (2007) Disruptive coloration in cuttlefish: a visual perception mechanism that regulates ontogenetic adjustment of skin patterning. Journal of Experimental Biology 210:1139-1147

Barnard K, Funt B (2002) Camera characterization for color research. Color Research \& Application 27 (3):152-163

Bass M, DeCusatis C, Enoch J, Lakshminarayanan V, Li G, Macdonald C, Mahajan V, Van Stryland E (2009) Handbook of optics, Volume II: Design, fabrication and testing, sources and detectors, radiometry and photometry. McGraw-Hill, Inc.,

Boggs CL, Watt WB, Ehrlich PR (2003) Butterflies: ecology and evolution taking flight. University of Chicago Press

Boycott, BB (1961) The functional organization of the brain of the cuttlefish Sepia officinalis, Proceedings of the Royal Society of London, Series B, Biological Sciences, Vol. 153, No. 953, 503-534.

Brainard D (2001) Color vision theory. International Encyclopedia of the Social and Behavioral Sciences, vol 4. Elsevier

Buresch KC, Mäthger LM, Allen JJ, Bennice C, Smith N, Schram J, Chiao CC, Chubb C, Hanlon RT (2011) The use of background matching vs. masquerade for camouflage in cuttlefish Sepia officinalis. Vision Research

Burt PJ, Adelson EH (1983) A multiresolution spline with application to image mosaics. ACM Trans Graph 2 (4):217-236. doi:10.1145/245.247

Bybee SM, Yuan F, Ramstetter MD, Llorente-Bousquets J, Reed RD, Osorio D, Briscoe AD (2012) UV photoreceptors and UV-yellow wing pigments in Heliconius butterflies allow a color signal to serve both mimicry and intraspecific communication. The American Naturalist 179 (1):38-51

Chakrabarti A, Scharstein D, Zickler T An empirical camera model for internet color vision. In: Proc. British Machine Vision Conference, 2009. BMVA Press, pp 51.51-51.11

Chang CI (2003) Hyperspectral Imaging. Kluwer Academic/Plenum Publishers,

Cheeseman P, Kelly J, Self M, Stutz J, Taylor W, Freeman D (1993) Autoclass: A bayesian classification system. In: Readings in knowledge acquisition and learning. Morgan Kaufmann Publishers Inc., pp 431-441

Cheney KL, Grutter AS, Marshall NJ (2008) Facultative mimicry: cues for colour change and colour accuracy in a coral reef fish. Proceedings of the Royal Society B: Biological Sciences 275 (1631):117-122

Cheney KL, Marshall NJ (2009) Mimicry in coral reef fish: how accurate is this deception in terms of color and luminance? Behavioral Ecology 20 (3):459-468

Cheung V, Westland S, Connah D, Ripamonti C (2004) A comparative study of the characterisation of colour cameras by means of neural networks and polynomial transforms. Coloration technology 120 (1):19-25

Cheung V, Westland S, Li C, Hardeberg J, Connah D (2005) Characterization of trichromatic color cameras by using a new multispectral imaging technique.

JOSA A 22 (7):1231-1240 
Chiao C-C, Chubb C, Buresch K, Siemann L (2009) The scaling effects of substrate texture on camouflage patterning in cuttlefish. Vision Research 49:1647-1656

Chiao C-C, Chubb C, Buresch KC, Barbosa A, Allen JJ, Mäthger LM, Hanlon RT (2010) Mottle camouflage patterns in cuttlefish: quantitative characterization and visual background stimuli that evoke them. Journal of Experimental Biology 213:187199

Chiao C-C, Chubb C, Hanlon RT (2007) Interactive effects of size, contrast, intensity and configuration of background objects in evoking disruptive camouflage in cuttlefish. Vision Research 47:2223-2235

Chiao C-C, Ulmer KM, Siemann LA, Buresch KC, Chubb C, Hanlon RT (2013) How visual edge features influence cuttlefish camouflage patterning. Vision Research $83,40-47$

Chiao CC, Hanlon RT (2001a) Cuttlefish camouflage: visual perception of size, contrast and number of white squares on artificial substrata initiates disruptive coloration. Journal of Experimental Biology 204:2119-2125

Chiao CC, Hanlon RT (2001b) Cuttlefish cue visually on area-not shape or aspect ratio-of light objects in the substrate to produce disruptive body patterns for camouflage. Biological Bulletin 201:269-270

Chiao CC, Kelman EJ, Hanlon RT (2005) Disruptive body patterning of cuttlefish (Sepia officinalis) requires visual information regarding edges and contrast of objects in natural substrate backgrounds. Biological Bulletin 208:7-11

Chiao CC, Osorio D, Vorobyev M, Cronin TW (2000) Characterization of natural illuminants in forests and the use of digital video data to reconstruct illuminant spectra. JOSA A 17 (10):1713-1721

Chiao CC, Wickiser JK, Allen JJ, Genter B, Hanlon RT (2011) Hyperspectral imaging of cuttlefish camouflage indicates good color match in the eyes of fish predators. Proceedings of the National Academy of Sciences 108 (22):9148-9153

Cortesi F, Cheney K (2010) Conspicuousness is correlated with toxicity in marine opisthobranchs. Journal of Evolutionary Biology 23 (7):1509-1518

Crook AC, Baddeley R, Osorio D (2002) Identifying the structure in cuttlefish visual signals. Philosophical Transactions of the Royal Society of London B 357 (1427): 1617-1624

da Fona Costa L, Cesar RM (2012) Shape Classification and Analysis: Theory and Practice, Second Edition. Taylor \& Francis

Davies DL, Bouldin DW (1979) A cluster separation measure. IEEE Transactions on Pattern Analysis and Machine Intelligence, (2):224-227

De La Barrera E, Smith WK (2009) Perspectives in Biophysical Plant Ecophysiology: A Tribute to Park S. Nobel.

Duda RO, Hart PE, Stork DG (2012) Pattern classification. John Wiley \& Sons,

Endler JA (1984) Progressive background in moths, and a quantitative measure of crypsis. Biological Journal of the Linnean Society 22 (3):187-231

Endler JA (1990) On the measurement and classification of colour in studies of animal colour patterns. Biological Journal of the Linnean Society 41 (4):315-352

Endler JA, Day LB (2006) Ornament colour selection, visual contrast and the shape of colour preference functions in great bowerbirds Chlamydera nuchalis Animal Behaviour 72 (6): 1405-1416 
Endler JA, Mielke Jr PW (2005) Comparing entire colour patterns as birds see them. Biological Journal of the Linnean Society 86 (4):405-431

Fairchild MD, Johnson GM METACOW: A public-domain, high-resolution, fully-digital, noise-free, metameric, extended-dynamic-range, spectral test target for imaging system analysis and simulation. In: Color and Imaging Conference, 2004. Society for Imaging Science and Technology, pp 239-245

Farid H (2011) That looks fake! http://www.fourandsix.com/blog/2011/6/29/that-looksfake.html.

Finkbeiner SD, Briscoe AD, Reed RD (2012) The benefit of being a social butterfly: communal roosting deters predation. Proceedings of the Royal Society B:

Biological Sciences 279 (1739):2769-2776

Finlayson G, Hordley S, Hubel PM Recovering device sensitivities with quadratic programming. In: IS\&T/SID Sixth Color Imaging Conference: Color Science, Systems and Applications, 1998.

Finlayson GD, Drew MS White-point preserving color correction. In: Color and Imaging Conference, 1997. Society for Imaging Science and Technology, pp 258-261

Florey E (1969) Ultrastructure and function of cephalopod chromatophores. American Zoologist:429-442

Freek Mvd (2006) The effectiveness of spectral similarity measures for the analysis of hyperspectral imagery. International Journal of Applied Earth Observation and Geoinformation 8 (1):3-17. doi:doi: 10.1016/j.jag.2005.06.001

Frey F, Farnand S (2011) Benchmarking Art Image Interchange Cycles. Final project report.

Godfrey D, Lythgoe J, Rumball D (1987) Zebra stripes and tiger stripes: the spatial frequency distribution of the pattern compared to that of the background is significant in display and crypsis. Biological Journal of the Linnean Society 32 (4):427-433

Grable MM, Shashar N, Gilles NL, Chiao C-C, Hanlon RT (2002) Cuttlefish body patterns as a behavioral assay to determine polarization perception. The Biological Bulletin 203 (2):232-234

Gurkan UA, Tasoglu S, Akkaynak D, Avci O, Unluisler S, Canikyan S, MacCallum N, Demirci U (2012) Smart Interface Materials Integrated with Microfluidics for OnDemand Local Capture and Release of Cells. Advanced healthcare materials 1 (5):661-668

Hanlon RT (2007) Cephalopod dynamic camouflage. Current Biology 17 (11):R400R405

Hanlon RT, Chiao C-C, Mäthger LM, Barbosa A, Buresch KC, Chubb C (2009) Cephalopod dynamic camouflage: bridging the continuum between background matching and disruptive coloration. Philosophical Transactions of the Royal Society of London B 364:429-437

Hanlon RT, Chiao C-C, Mäthger LM, Marshall NJ (2013) A fish-eye view of cuttlefish camouflage using in situ spectrometry. Biological Journal of the Linnean Society 109 (3):535-551. doi:10.1111/bij.12071

Hanlon RT, Messenger JB (1988) Adaptive coloration in young cuttlefish (Sepia officinalis L.): the morphology and development of body patterns and their 
relation to behaviour. Philosophical Transactions of the Royal Society of London B 320:437-487

Hanlon RT, Messenger JB (1996) Cephalopod Behaviour. . Cambridge University Press, Cambridge,

Hart N, Partridge J, Cuthill I (1998) Visual pigments, oil droplets and cone photoreceptor distribution in the European starling (Sturnus vulgaris). The Journal of experimental biology 201 (9):1433-1446

Hauglund K, Hagen SB, Lampe HM (2006) Responses of domestic chicks (Gallus gallus domesticus) to multimodal aposematic signals. Behavioral Ecology 17 (3):392398

Hedley JD, Roelfsema CM, Phinn SR, Mumby PJ (2012) Environmental and Sensor Limitations in Optical Remote Sensing of Coral Reefs: Implications for Monitoring and Sensor Design. Remote Sensing 4 (1):271-302

Heiling AM, Herberstein ME, Chittka L (2003) Pollinator attraction:Crab-spiders manipulate flower signals. Nature 421 (6921):334

Hochberg EJ, Atkinson MJ, Apprill A, Andrefouet S (2004) Spectral reflectance of coral. Coral Reefs 23 (1):84-95

Hong G, Luo MR, Rhodes PA (2001) A study of digital camera colorimetric characterisation based on polynomial modelling. Color Research \& Application 26 (1):76-84

Isaac LA, Gregory PT (2013) Can snakes hide in plain view? Chromatic and achromatic crypsis of two colour forms of the Western Terrestrial Garter Snake (Thamnophis elegans). Biological Journal of the Linnean Society 108 (4):756-772

Jerlov NG (1968) Optical Oceanography. Elsevier

Jerlov NG (1976) Marine Optics. Elsevier, Amsterdam

Jiang J, Liu D, Gu J, Süsstrunk S What is the Space of Spectral Sensitivity Functions for Digital Color Cameras? In: IEEE Workshop on the Applications of Computer Vision, Clearwater Beach, FL USA, 2013. pp 168-179

Johnson T (1996) Methods for characterizing colour scanners and digital cameras. Displays 16 (4):183-191

Joshi N, Jensen H (2004) Color calibration for arrays of inexpensive image sensors. Citeseer

Julesz B (1962) Visual pattern discrimination. Information Theory, IRE Transactions on 8 (2):84-92

Kelber A, Vorobyev M, Osorio D (2003) Animal colour vision, behavioural tests and physiological concepts. Biological Reviews 78 (1):81-118

Kelman EJ, Baddeley RJ, Shohet AJ, Osorio D (2007) Perception of visual texture and the expression of disruptive camouflage by the cuttlefish, Sepia officinalis. Proceedings of the Royal Society B 274 (1616):1369-1375. doi:10.1098/rspb.2007.0240

Kelman EJ, Osorio D, Baddeley R (2008) A review of cuttlefish camouflage and object recognition and evidence for depth perception. Journal of Experimental Biology 211:1757-1763

King RB (1992) Lake Erie water snakes revisited: morph-and age-specific variation in relative crypsis. Evolutionary Ecology 6 (2):115-124 
Kruse FA, Lefkoff AB, Boardman JW, Heidebrecht KB, Shapiro AT, Barloon PJ, Goetz AFH (1993) The spectral image processing system (SIPS) - interactive visualization and analysis of imaging spectrometer data. Remote Sensing of Environment 44 (2-3):145--163. doi:doi: 10.1016/0034-4257(93)90013-N

Langmore N, Stevens M, Maurer G, Kilner R (2009) Are dark cuckoo eggs cryptic in host nests? Animal Behaviour 78 (2):461-468

Langridge KV (2006) Symmetrical crypsis and asymmetrical signalling in the cuttlefish Sepia officinalis. Proceedings of the Royal Society B 273:959-967

Lee Y-H, Yan HY, Chiao C-C (2010) Visual contrast modulates maturation of camouflage body patterning in cuttlefish (Sepia pharaonis). Journal of Comparative Psychology 124 (3):261

Leiper I, Phinn S, Dekker AG (2012) Spectral reflectance of coral reef benthos and substrate assemblages on Heron Reef, Australia. International Journal of Remote Sensing 33 (12):3946-3965

Levin N, Ben-Dor E, Singer A (2005) A digital camera as a tool to measure colour indices and related properties of sandy soils in semi-arid environments. International Journal of Remote Sensing 26 (24):5475-5492

Lim A, Hedley JD, LeDrew E, Mumby PJ, Roelfsema C (2009) The effects of ecologically determined spatial complexity on the classification accuracy of simulated coral reef images. Remote Sensing of Environment 113 (5):965-978

Lind O, Mitkus M, Olsson P, Kelber A (2013) Ultraviolet sensitivity and colour vision in raptor foraging. The Journal of Experimental Biology 216 (10):1819-1826

Luo MR, Cui G, Rigg B (2001) The development of the CIE 2000 colour-difference formula: CIEDE2000. Color Research \& Application 26 (5):340-350. doi:10.1002/col.1049

Maan ME, Cummings ME (2008) Female preferences for aposematic signal components in a polymorphic poison frog. Evolution 62 (9):2334-2345

Maan ME, Cummings ME (2012) Poison frog colors are honest signals of toxicity, particularly for bird predators. The American Naturalist 179 (1):E1-E14

Marshall N, Jennings K, McFarland W, Loew E, Losey G (2003a) Visual biology of Hawaiian coral reef fishes. II. Colors of Hawaiian coral reef fish. Journal Information 2003 (3)

Marshall N, Jennings K, McFarland W, Loew E, Losey G (2003b) Visual biology of Hawaiian coral reef fishes. III. Environmental light and an integrated approach to the ecology of reef fish vision. Journal Information 2003 (3)

Marshall NJ, Messenger JB (1996) Colour-blind camouflage. Nature 382:408-409

Marshall NJ, Vorobyev M (2003) The design of color signals and color vision in fishes. Sensory Processing in Aquatic Environments: 194-222

Mäthger LM, Barbosa A, Miner S, Hanlon RT (2006) Color blindness and contrast perception in cuttlefish (Sepia officinalis) determined by a visual sensorimotor assay. Vision Research 46:1746-1753

Mäthger LM, Chiao C-C, Barbosa A, Hanlon RT (2008) Color matching on natural substrates in cuttlefish, Sepia officinalis. J Comp Physiol A 194:577-585

Mäthger LM, Chiao CC, Barbosa A, Buresch KC, Kaye S, Hanlon RT (2007) Disruptive coloration elicited on controlled natural substrates in cuttlefish, Sepia officinalis. Journal of Experimental Biology 210:2657-2666 
Mäthger LM, Denton EJ, Marshall NJ, Hanlon RT (2009) Mechanisms and behavioural functions of structural coloration in cephalopods. Journal of the Royal Society Interface 6:S149-S163

Matz MV, Marshall NJ, Vorobyev M (2006) Are corals colorful? Photochemistry and Photobiology 82 (2):345-350

Mauer C, Wueller D Measuring the spectral response with a set of interference filters. In: IS\&T/SPIE Electronic Imaging, 2009. International Society for Optics and Photonics, pp 72500S-72500S-72510

McKay BD (2013) The use of digital photography in systematics. Biological Journal of the Linnean Society 110 (1):1-13. doi:10.1111/bij.12086

Messenger J (1974) Reflecting elements in cephalopod skin and their importance for camouflage. Journal of Zoology 174 (3):387-395

Messenger J (2001a) Cephalopod chromatophores: neurobiology and natural history. Biological Reviews 76 (4):473-528

Messenger JB (2001b) Cephalopod chromatophores: neurobiology and natural history. Biological Reviews 76:473-528

Nakamura J (2005) Image Sensors and Signal Processing for Digital Still Cameras. Taylor \& Francis

Nidamanuri RR, Zbell B (2011) Normalized Spectral Similarity Score NS3 as an Efficient Spectral Library Searching Method for Hyperspectral Image Classification. Selected Topics in Applied Earth Observations and Remote Sensing, IEEE Journal of 4 (1):226-240

Nokelainen O, Hegna RH, Reudler JH, Lindstedt C, Mappes J (2012) Trade-off between warning signal efficacy and mating success in the wood tiger moth. Proceedings of the Royal Society B: Biological Sciences 279 (1727):257-265

Packard A (1972) Cephalopods and fish: the limits of convergence. Biological Reviews 47:241-307

Packard A, Hochberg F (1977) Skin patterning in Octopus and other genera. In: Symp. Zool. Soc. Lond. pp 191-231

Pharr M, Humphreys G (2010) Physically based rendering: From theory to implementation. Morgan Kaufmann,

Pike TW (2011a) Generalised chromaticity diagrams for animals with n-chromatic colour vision. Journal of Insect Behavior:1-10

Pike TW (2011b) Using digital cameras to investigate animal colouration: estimating sensor sensitivity functions. Behavioral Ecology and Sociobiology 65 (4):849-858

Ramanath R, Snyder WE, Bilbro GL, Sander WA (2002) Demosaicking methods for Bayer color arrays. Journal of Electronic imaging 11 (3):306-315

Ravishankar Rao A, Lohse GL (1996) Towards a texture naming system: Identifying relevant dimensions of texture. Vision Research 36 (11):1649-1669. doi:http://dx.doi.org/10.1016/0042-6989(95)00202-2

Reinhard E, Khan E, Akyüz A, Johnson G (2008) Color imaging: fundamentals and applications. AK Peters, Ltd

Roelfsema C, Marshall NJ, Hochberg EJ, Phinn S, Goldizen A, Gill T (2006) Underwater Spectrometer System (UWSS04). 
Rosen MR, Frey FS (2005) RIT American museums survey on digital imaging for direct capture of artwork. In: Society for Imaging Science and Technology Archiving Conference Springfield, VA USA.

Seon Joo K, Hai Ting L, Zheng L, Su, x, sstrunk S, Lin S, Brown MS (2012) A New InCamera Imaging Model for Color Computer Vision and Its Application. Pattern Analysis and Machine Intelligence, IEEE Transactions on 34 (12):2289-2302. doi:10.1109/tpami.2012.58

Shand J, Archer MA, Collin SP (1999) Ontogenetic changes in the retinal photoreceptor mosaic in a fish, the black bream, Acanthopagrus butcheri. The Journal of comparative neurology 412 (2):203-217

Sharma G, Wu W, Dalal EN (2005) The CIEDE2000 color-difference formula: Implementation notes, supplementary test data, and mathematical observations. Color Research \& Application 30 (1):21-30. doi:10.1002/col.20070

Shirley P, Ashikhmin M, Marschner S (2009) Fundamentals of computer graphics. CRC Press

Shohet AJ, Baddeley RJ, Anderson JC, Kelman EJ, Osorio D (2006) Cuttlefish responses to visual orientation of substrates, water flow and a model of motion camouflage. Journal of Experimental Biology 209:4717-4723

Shohet AJ, Baddeley RJ, Anderson JC, Osorio D (2007) Cuttlefish camouflage: a quantitative study of patterning. Biological Journal of the Linnean Society 92:335-345

Siddiqi A, Cronin TW, Loew ER, Vorobyev M, Summers K (2004) Interspecific and intraspecific views of color signals in the strawberry poison frog Dendrobates pumilio. Journal of Experimental Biology 207 (14):2471-2485

Siebeck U, Marshall N, Klüter A, Hoegh-Guldberg O (2006) Monitoring coral bleaching using a colour reference card. Coral Reefs 25 (3):453-460

Skelhorn J, Rowland HM, Ruxton GD (2010) The evolution and ecology of masquerade. Biological Journal of the Linnean Society 99:1-8

Skelhorn J, Ruxton GD (2011) Mimicking multiple models: polyphenetic masqueraders gain additional benefits from crypsis. Behavioral Ecology 22 (1):60-65

Smith RC, Baker KS (1978) Optical classification of natural waters. Limnology and Oceanography 23 (2)

Spottiswoode CN, Stevens M (2012) Host-parasite arms races and rapid changes in bird egg appearance. The American Naturalist 179 (5):633-648

Stevens M (2011) Avian vision and egg colouration: Concepts and measurements. Avian Biology Research 4 (4):168-184

Stevens M, Merilaita S (2009) Animal camouflage: current issues and new perspectives. Philosophical Transactions of the Royal Society of London B 364:423-427

Stevens M, Parraga CA, Cuthill IC, Partridge JC, Troscianko TS (2007) Using digital photography to study animal coloration. Biological Journal of the Linnean Society 90 (2):211-237

Stoddard MC, Marshall KL, Kilner RM (2011) Imperfectly camouflaged avian eggs: artefact or adaptation? Avian Biology Research 4 (4):196-213

Stoddard MC, Stevens M (2011) Avian vision and the evolution of egg color mimicry in the common cuckoo. Evolution 65 (7):2004-2013 
Stuart-Fox D, Moussalli A (2008) Selection for social signalling drives the evolution of chameleon colour change. PLoS biology 6 (1):e25

Süsstrunk SE, Holm JM, Finlayson GD Chromatic adaptation performance of different RGB sensors. In: Photonics West 2001-Electronic Imaging, 2000. International Society for Optics and Photonics, pp 172-183

Sweet JN (2003) The spectral similarity scale and its application to the classification of hyperspectral remote sensing data. Advances in Techniques for Analysis of Remotely Sensed Data, 2003 IEEE Workshop on:92-99

Szeliski R (2010) Computer vision: algorithms and applications. Springer,

Théry M, Casas J (2002) Predator and prey views of spider camouflage. Nature 415 (6868):133

Tyler JE, Smith RC (1970) Measurements of spectral irradiance underwater, vol 1. Gordon \& Breach Publishing Group

Ulmer KM, Buresch KC, Kossodo M, Mäthger L, Siemann LA, Hanlon RT (2013) Vertical visual features have a strong influence on cuttlefish camouflage. The Biological Bulletin 224 (2):110-118

Vasilescu I (2009) Using light underwater: devices, algorithms and systems for maritime persistent surveillance. Massachusetts Institute of Technology

Vorobyev M, Osorio D (1998) Receptor noise as a determinant of colour thresholds. Proceedings of the Royal Society B: Biological Sciences 265 (1394):351-358

Wardill TJ, Gonzalez-Bellido PT, Crook RJ, Hanlon RT (2012) Neural control of tuneable skin iridescence in squid. Proceedings of the Royal Society B: Biological Sciences 279 (1745):4243-4252

Wee AG, Lindsey DT, Kuo S, Johnston WM (2006) Color accuracy of commercial digital cameras for use in dentistry. Dental materials: official publication of the Academy of Dental Materials 22 (6):553

Westland S, Ripamonti C (2004) Computational Colour Science Using MATLAB. Wiley Online Library

Winters G, Holzman R, Blekhman A, Beer S, Loya Y (2009) Photographic assessment of coral chlorophyll contents: Implications for ecophysiological studies and coral monitoring. Journal of Experimental Marine Biology and Ecology 380 (1):25-35

Wyszecki G, Stiles WS (2000) Color science: concepts and methods, quantitative data, and formulae. 2, illustrated edn. John Wiley \& Sons

Yuhas RH, Goetz AFH, Boardman JW Discrimination among semi-arid landscape endmembers using the spectral angle mapper (SAM) algorithm. In, 1992.

Pasadena, CA: JPL Publication, pp 147-149

Zylinski S, Darmaillacq A-S, Shashar N (2012) Visual interpolation for contour completion by the European cuttlefish (Sepia officinalis) and its use in dynamic camouflage. Proceedings of the Royal Society B: Biological Sciences 279 (1737):2386-2390

Zylinski S, How M, Osorio D, Hanlon RT, Marshall N (2011) To be seen or to hide: visual characteristics of body patterns for camouflage and communication in the Australian giant cuttlefish Sepia apama. The American Naturalist 177 (5):681690

Zylinski S, Osorio D, Shohet AJ (2009a) Cuttlefish camouflage: context-dependent body pattern use during motion. Proceedings of the Royal Society B 276:3963-3969 
Zylinski S, Osorio D, Shohet AJ (2009b) Edge detection and texture classification by cuttlefish. Journal of Vision 9 (13):1-10

Zylinski S, Osorio D, Shohet AJ (2009c) Perception of edges and visual texture in the camouflage of the common cuttlefish Sepia officinalis. Philosophical Transactions of the Royal Society B 364:439-448 